\title{
Light passing through subwavelength apertures
}

\author{
F. J. Garcia-Vidal \\ Departamento de Física Teórica de la Materia Condensada, Universidad Autónoma de \\ Madrid, E-28049 Madrid, Spain

\section{Martin-Moreno} \\ Instituto de Ciencia de Materiales de Aragón (ICMA) and Departamento de Física de la \\ Materia Condensada, CSIC-Universidad de Zaragoza, E-50009 Zaragoza, Spain
}

T. W. Ebbesen

ISIS, Université de Strasbourg and CNRS, 67000 Strasbourg, France

\section{Kuipers}

Center for Nanophotonics, FOM Institute for Atomic and Molecular Physics, Science Park 104, 1098 XG Amsterdam, The Netherlands

(Published 12 March 2010)

\begin{abstract}
This review provides a perspective on the recent developments in the transmission of light through subwavelength apertures in metal films. The main focus is on the phenomenon of extraordinary optical transmission in periodic hole arrays, discovered over a decade ago. It is shown that surface electromagnetic modes play a key role in the emergence of the resonant transmission. These modes are also shown to be at the root of both the enhanced transmission and beaming of light found in single apertures surrounded by periodic corrugations. This review describes both the theoretical and experimental aspects of the subject. For clarity, the physical mechanisms operating in the different structures considered are analyzed within a common theoretical framework. Several applications based on the transmission properties of subwavelength apertures are also addressed.
\end{abstract}

DOI: 10.1103/RevModPhys.82.729

PACS number(s): 42.79.Gn, 73.20.Mf, 42.25.Bs

\section{CONTENTS}

I. Introduction

A. Theoretical methods

II. Transmission through Single Apertures

A. One-dimensional aperture: Single slit

B. Two-dimensional single apertures

1. Circular holes

2. Rectangular holes

III. Transmission through Arrays of Apertures

A. One-dimensional periodic arrays of slits

1. Theoretical results

2. Coupled-mode analysis

3. Experimental results

B. Two-dimensional periodic arrays of holes

1. General results

2. Coupled-mode analysis

3. Dependence on the material properties

4. Size and shape dependence

5. Electric field enhancement and nonlinear effects

6. Finite-size effects

7. Polarization effects

8. Dynamics of the EOT phenomenon

C. Quasiperiodic arrays

1. General results

2. Coupled-mode analysis

IV. Transmission through Single Apertures Flanked by
Periodic Corrugations $\quad 764$

A. General results 764

B. Coupled-mode analysis 767

V. Applications 770

A. Molecular sensing and spectroscopy 771

1. SPR 771

2. Enhanced absorption $\quad 771$

3. Fluorescence 772

B. Photonic devices and methods 772

VI. Conclusions and Perspectives 774 Acknowledgments 775

Appendix: Coupled-Mode Method $\quad 775$

1. Periodic systems 775

2. Multiple scattering $\quad 777$

3. Finite systems 777

4. Equations in Fourier space 779

5. Bound modes in holey PEC surfaces and films 779

References

780

\section{INTRODUCTION}

The optical properties of subwavelength apertures in metallic films have been the focus of much research activity around the world since the extraordinary optical transmission (EOT) phenomenon was reported over a decade ago (Ebbesen et al., 1998). The aim of this paper is to review this extensive work. We consider the fundamental aspects of the phenomenon by comparing experimental findings with theoretical analysis. We also discuss 
several applications based on EOT that have already been reported. Additionally, for clarity, this review provides a unified view of the transmission properties of structures composed of subwavelength apertures by presenting the results obtained within the same theoretical framework, a coupled-mode method.

Extraordinary optical transmission is an optical phenomenon in which a structure containing subwavelength apertures in an opaque screen transmits more light than might naively be expected on the basis of either ray optics or even knowledge of the transmission through individual apertures. The phenomenon was discovered serendipitously for two-dimensional (2D) periodic arrays of subwavelength holes in metals (Ebbesen et al., 1998). In the original experiments, the holes were milled in optically thick metal films and, therefore, the electromagnetic (EM) waves could only tunnel through the apertures in the transmission process. Surprisingly, such arrays may, for certain wavelengths, exhibit transmission efficiencies (normalized to the total area of the holes) that exceed unity. In other words, for these wavelengths a periodic array of subwavelength holes transmits more light than a large macroscopic hole with the same area as the sum of all the small holes. The surprise is compounded by the fact that a single subwavelength aperture generally transmits light with an efficiency that is substantially below unity. This low transmission efficiency is due to both the poor coupling of subwavelength holes to radiative EM modes (Bethe, 1944) and the evanescent decay of the EM fields inside the holes (Roberts, 1987). Clearly, in the EOT phenomenon the holey metal film is not merely a screen that blocks the light, but rather an active participant in the transmission process. In physical terms, a collective response of the periodic array of holes must occur in order to boost the transmission. There have been different proposals regarding the physical origin of this collective behavior, which will be discussed in this review. However, there is now a wide consensus regarding the key role played by surface EM modes in the appearance of EOT.

In order to place EOT in a historical perspective, it must be noted that very thin metal films perforated with 2D hole arrays had been extensively studied before 1998 mainly due to their applications as selective filters [see, e.g., Munk (2000)]. Even one-dimensional (1D) mesh filters were already in use in the 19th century for microwaves, most notably by Bose in Calcutta (Emerson, 1997). In the 2D case, the high-pass filtering properties are provided by the aforementioned low transmission efficiency for wavelengths longer than the cutoff wavelength. The low-pass filtering arises from the redistribution of energy caused by the periodic array when a new diffraction order begins to propagate, therefore carrying away energy at angles different from the incident one. More precisely, hole arrays were known to act as bandpass filters for $d<\lambda<\lambda_{C}$, where $\lambda_{C}$ is the hole cutoff wavelength and $d$ is the lattice parameter. However, the EOT phenomenon presents three main differences with respect to the previous work. First, the original experiments were performed in the optical regime, where

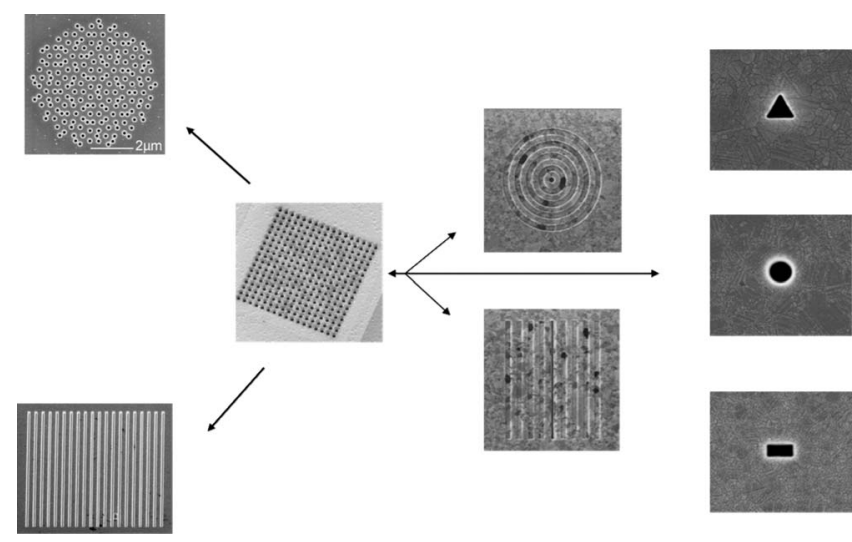

FIG. 1. Various types of aperture structures that are considered in this review.

there are EM modes bound to the metal surface known as surface plasmon polaritons (SPPs), which could play an important role in the transmission process [SPPs were discovered by Ritchie (1957); for a recent review, see Pitarke et al. (2007)]. Second, the perforated films in EOT are usually optically thick, i.e., much larger than the skin depth of the metal. Third, the geometrical parameters defining the EOT structures were such that $\lambda_{C} \leqslant d<\lambda$, i.e., the holes were at cutoff when EOT peaks occurred. This is not a minor point as, combined with the weak coupling of subwavelength holes to radiation modes, it is responsible for the appearance of welldefined narrow resonant peaks.

Over the years EOT has been expanded by the creation of single apertures surrounded by periodic structures to efficiently "harvest" light and subsequently squeeze it through the apertures (Fig. 1). The metal can be sculpted not only on the incident surface to control the coupling of the incident light and its scattering dynamics at the surface but also on the exit surface to modify the reemission pattern emerging from the aperture, leading to the beaming phenomenon (Lezec et al., 2002).

Interest in the EOT process arises in part because the structures provide high contrast between the opaque metal and the bright subwavelength aperture(s), be there one or many. Moreover, as shown in this review, EOT offers the prospect of a multitude of applications as the remarkably high transmission efficiencies and, concomitantly, high local field enhancements can be achieved at wavelengths that can be engineered through the geometry of the metal surface.

Because of its link with the excitation of surface plasmons at corrugated metal surfaces, a general overview of the EOT process has already been presented in recent review articles (Barnes et al., 2003; Ozbay, 2006) or in books (Maier, 2007) dedicated to surface plasmon photonics. There have been also some recent papers specifically devoted to describing different aspects of the EOT phenomenon. Genet and Ebbesen (2007) aimed to present this phenomenon and its main applications to a general audience; the spectroscopic capabilities of holey metal films have also been the subject of several detailed 
reviews (Coe et al., 2006, 2008; Gordon et al., 2008; Sinton et al., 2008). On the other hand, in a very recent Colloquium paper published in this journal, De Abajo (2007) focused on presenting a tutorial analysis of the interaction of light with $2 \mathrm{D}$ periodic arrays of holes or metallic particles.

In this review, we give a complete and detailed overview of the EOT phenomenon by presenting (i) a summary of the different EOT processes emerging in 1D and $2 \mathrm{D}$ structures, (ii) a compilation of the research done in EOT from both the experimental and theoretical sides, and (iii) a unified view of all considered phenomena using the same theoretical method. The structure of the paper is as follows. In the Introduction, we present a description of the different theoretical frameworks that have been used to study the transmission of light through subwavelength apertures. A general overview of the coupled-mode method is also presented. Section II reviews the optical properties of individual apertures in order to better separate the effects due to the periodic arrangement from those already present in isolated apertures. The emergence of EOT in periodic arrays of subwavelength apertures, in both $1 \mathrm{D}$ and $2 \mathrm{D}$ structures, is discussed in Sec. III. The case of a single subwavelength aperture surrounded by periodic corrugations, leading to enhanced transmission and beaming effects, is analyzed in Sec. IV. In all those sections, we compare theoretical analysis and the experimental results. In Sec. V, we discuss several EOT applications that have been investigated, in particular, in the area of spectroscopy and molecular probes, as well as stand-alone photonic devices. Finally, in Sec. VI, we consider the extension of these ideas to other types of waves and speculate about future trends. The technical details of the coupled-mode method are presented in the Appendix.

\section{A. Theoretical methods}

In what follows we summarize the theoretical approaches that have been used to study optical transmission through subwavelength apertures. Although the equations that govern electromagnetic phenomena in metallodielectric structures are well established (the macroscopic Maxwell equations plus the constitutive relations describing material properties), it is a difficult task to solve them because of the highly different length scales present in metals. In the optical regime, important roles are simultaneously played by the free-space wavelength $\lambda(400-800 \mathrm{~nm})$, the skin depth of the metal (of the order of $30 \mathrm{~nm}$ ), the dimensions of the scatterers placed on the metal (tens to hundreds of nanometers), the typical length scale associated with their geometrical arrangement (hundreds of nanometers to a couple of microns), and the device dimensions (several microns). Several methods have been used to compute the transmission of EM fields through apertures, each one presenting its own advantages and disadvantages. In general, no computational tool is best in all circumstances.
The theoretical study of single apertures in a real metal has been treated by a variety of methods such as the multiple-multipole (MMP) method (Wannemacher, 2001), Green's dyadic method (GDM) (Colas des Francs et al., 2005; Sepúlveda et al., 2008), and the finitedifference time domain (FDTD) method (Chang et al., 2005; Popov et al., 2005).

The first two methods are computationally demanding (the MMP method due to the large number of multipoles needed in order to achieve convergence, and the GDM due to the difficulty in evaluating the Sommerfeld integrals involved). The FDTD method is faster, but its applicability is sometimes limited by the small grid sizes needed to reproduce the rapid variations of the EM fields at metal-dielectric interfaces. Additionally, some technical problems due to the finite simulation window must be overcome (for instance, the implementation of plane-wave illumination in finite systems is not trivial).

The theoretical analysis of the optical response of arrays of apertures is greatly simplified if the arrays are considered infinite and periodic. Then, with the help of Bloch's theorem, only EM fields within one unit cell need be computed. This allows for accurate calculation of the transmission properties of arrays of apertures using different numerical techniques:

- The rigorous coupled-wave method (RCWM) (Li, 1997), based on the modal expansion of the fields, in which the eigenmodes in the perforated metal film are computed after expansion of the dielectric constant in harmonics (Popov et al., 2000; Lalanne, Rodier, and Hugonin, 2005). A modification of this general method has recently been used to also study single apertures (Lalanne, Hugonin, and Rodier, 2005).

- A differential method (Salomon et al., 2001), borrowed from the traditional method for analyzing metallic gratings. This has been used to study extremely thin films (metal thickness of the order of $20 \mathrm{~nm}$ ).

- The transfer matrix method (Pendry and MacKinnon, 1992; Bell et al., 1995), in which the EM fields are discretized in space at a given frequency. This method is computationally demanding and has only been applied to 1D systems (Porto et al., 1999).

- The FDTD method (Baida and Van Labeke, 2003; Müller et al., 2004; Miyamaru and Hangyo, 2005; Chen et al., 2008; Rodrigo et al., 2008).

A number of approximate techniques have also been used. One of them is based on the RCWM but considers only a few harmonics in the expansion for the dielectric constant of the metal (Dykhne et al., 2003; Darmanyan et al., 2004; Kats and Nikitin, 2004; Kats et al., 2005). The advantages of this method are that it can be worked out analytically and can treat resonant optical transmission through corrugated continuous thin metal films (Gruhlke et al., 1986; Chen et al., 2008).

Computing the optical properties of arrays of apertures beyond these two limiting cases (isolated apertures 
and infinitely periodic arrays) is much more difficult and the calculation must, in general, be done with approximate methods. Collin and Eggimann (1961) developed a formalism valid for extremely small circular holes $(\lambda \gg 2 \pi r$, where $r$ is the radius of the hole) based on the coupling of the electric and magnetic dipoles that represent the hole in this limit (Bethe, 1944). In its original formulation, the method could only treat holes in an impenetrable screen of zero thickness. Recently, the extension to metal films of finite thickness has been presented (De Abajo et al., 2005; De Abajo, 2007). Another method, which can be considered as an extension of the RCWM, considers the hole as a finite portion of a finite waveguide; correspondingly, the EM fields inside the perforated metal are expanded in terms of waveguide modes. This modal expansion approach is not restricted to either small holes or perfect conductors and is valid when all distances between apertures and the thickness of the metal film are a few times the skin depth of the metal (i.e., when the apertures cannot interact across the bulk of the metal).

Application of modal expansion methods to analyze the transmission properties of $1 \mathrm{D}$ or $2 \mathrm{D}$ gratings began in the 1960s. One of the first studies was carried out by Galindo and $\mathrm{Wu}$ (1966), who presented numerical solutions for an infinite array of rectangular holes drilled in extremely thin metal films. In a series of papers (Chen, 1970, 1971, 1973), Chen rigorously formulated the basic equations for analyzing infinitely periodic $2 \mathrm{D}$ arrays of circular or rectangular holes drilled in both thin and thick films. This formulation was the basis for many numerical calculations aiming to analyze the transmission properties of metallic wire meshes (i.e., holes occupying most of the area of the unit cell) (McPhedran and Maystre, 1977; Bliek et al., 1980; Botten et al., 1985).

The theoretical formalism used throughout this review, called the coupled-mode method, is included in this modal expansion category. We present here an overview of this method, as its derivation, notation, and, more importantly, its range of applicability (it applies for single apertures and both finite and infinitely periodic arrays of apertures) are different from those in the aforementioned references. In this section we present its basic ingredients; in order to improve the readability, we leave the detailed derivations for the Appendix. An important asset of the coupled-mode method is that the same equations can deal with the physics of very different structures. In a first approximation, the metal is treated as a perfect electrical conductor (PEC), i.e., $\epsilon$ $=-\infty$. The main advantage of this approximation is that the EM modes inside the apertures (slits or holes) coincide with the waveguide modes of the apertures, which are known analytically for some geometries. This PEC model is quasiexact for metals in the terahertz or microwave range of the EM spectrum. Moreover, the finite dielectric constant of metals at optical frequencies can be approximately incorporated into the formalism using the surface impedance boundary conditions (SIBCs) (Landau et al., 1960). Within this approach, the coupledmode method also has semiquantitative value in the op-

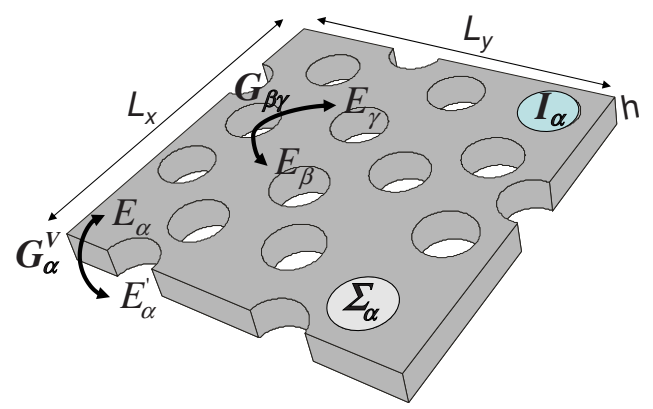

FIG. 2. (Color online) Schematic representation of a unit cell of lengths $L_{x}$ and $L_{y}$ containing a finite set of indentations perforated on a metallic film of thickness $h$. The different terms appearing in the set of equations (1) are also schematically depicted.

tical regime for good conductors such as silver or gold.

Figure 2 is a schematic of the general system under study: a set of apertures or indentations placed on a metallic film of thickness $h$. In this section we present the general formalism for the 2D geometry comprising a set of holes and/or dimples. The implementation for the 1D case (slits and/or grooves) is straightforward. This set can be formed by any combination of holes and/or indentations. In all these structures, the system can be divided into three regions. Regions I (reflection) and III (transmission) are dielectric semispaces characterized by positive dielectric constants $\epsilon_{1}$ and $\epsilon_{3}$, respectively. Region II represents the perforated metal film with a frequency-dependent dielectric function $\epsilon_{M}(\omega)$. We assume that the structure is illuminated by EM plane waves coming from region I. Nevertheless, arbitrary illumination could be included straightforwardly, which is another strength of the method.

We expand the EM fields on the eigenmodes of each region (EM plane waves in regions I and III and waveguide modes inside the apertures) and match the parallel components of the electric and magnetic fields at the two interfaces $(z=0$ and $h)$. EM fields in regions I and III are expanded in terms of plane waves characterized by their parallel momentum $\vec{k}$ and polarization $\sigma$. For the modal expansion of the EM fields within the apertures, we use a compact notation, denoting as "object" $\alpha$ any waveguide EM mode considered in the expansion. Therefore, an object is defined by (i) the indentation it belongs to, (ii) its polarization (TM or TE modes), and (iii) the indices related to its spatial dependence. Finally in the formalism, all that needs to be known are the parallel components of the $E$ field associated with the corresponding waveguide mode and its propagation constant $q_{z \alpha}$.

After some algebraic manipulations, we end up with a coupled system of equations for $E_{\alpha}$ and $E_{\alpha}^{\prime}$ (which are the modal amplitudes of the electric field at the input and output sides of the apertures, respectively): 


$$
\begin{aligned}
& \left(G_{\alpha \alpha}-\Sigma_{\alpha}\right) E_{\alpha}+\sum_{\beta \neq \alpha} G_{\alpha \beta} E_{\beta}-G_{\alpha}^{V} E_{\alpha}^{\prime}=I_{\alpha}, \\
& \left(G_{\gamma \gamma}^{\prime}-\Sigma_{\gamma}\right) E_{\gamma}^{\prime}+\sum_{\nu \neq \gamma} G_{\gamma \nu}^{\prime} E_{\nu}^{\prime}-G_{\gamma}^{V} E_{\gamma}=0 .
\end{aligned}
$$

This is the basic set of linear equations that will be used throughout to describe the transmission properties of different structures containing subwavelength apertures. Details of the derivation, as well as the mathematical expressions for the different magnitudes, can be found in the Appendix. In this section we simply give the physical meaning.

- The independent term $I_{\alpha}$ takes into account the direct initial illumination over object $\alpha$.

- $\Sigma_{\alpha}$ is related to the bouncing back and forth of the EM fields inside object $\alpha$.

- $G_{\alpha}^{V}$ accounts for the coupling between the EM fields at the two sides of the aperture.

- The term $G_{\alpha \beta}$ controls the EM coupling between objects $\alpha$ and $\beta$ in region I. This "propagator" takes into account that each point in object $\beta$ emits EM radiation, which can be "collected" by object $\alpha$. The propagator $G_{\gamma \nu}^{\prime}$ differs from $G_{\alpha \beta}$ only in the constituents; i.e., $G_{\gamma \nu}^{\prime}$ is a function of $\epsilon_{3}$, whereas $G_{\alpha \beta}$ depends on $\epsilon_{1}$.

Formally the propagator $G_{\alpha \beta}$ can be written as

$$
G_{\alpha \beta}=i \sum_{\vec{k} \sigma} Y_{\vec{k} \sigma}\langle\alpha \mid \vec{k} \sigma\rangle\langle\vec{k} \sigma \mid \beta\rangle
$$

with a similar expression for $G_{\alpha \beta}^{\prime}$. In Eq. (2), $Y_{\vec{k} \sigma}$ is the admittance of the plane wave: $Y_{\vec{k} s}=k_{z} / k_{\omega}$ and $Y_{\vec{k} p}$ $=\epsilon_{1} k_{\omega} / k_{z}$ for $s$ and $p$ polarizations, respectively, with $k_{\omega}=\omega / c=2 \pi / \lambda$ ( $\omega$ is the frequency and $c$ is the speed of light) and $|k|^{2}+k_{z}^{2}=k_{\omega}^{2}$. The overlap integral between mode $\alpha$ and plane wave $\vec{k} \sigma$ is written in Eq. (2) as $\langle\alpha \mid \vec{k} \sigma\rangle$ and its detailed expression can be found in Eq. (A7).

As stated above, the finite conductivity of the metal can be incorporated into this coupled mode framework by introducing SIBCs instead of perfect conductor boundary conditions. Within this approximation, the set of equations (1) is still valid but with magnitudes that now depend on the surface impedance of the metal, $Z_{S}$ $=1 / \sqrt{\epsilon_{M}(\omega)}$. Expressions for $I_{\alpha}, G_{\alpha}^{V}, \Sigma_{\alpha}$, and $G_{\alpha \beta}$ within the SIBC approach can also be found in the Appendix.

The EM field everywhere in space can be obtained from the modal amplitudes $\left\{E_{\alpha}, E_{\alpha}^{\prime}\right\}$. Therefore, the coupled-mode method reduces the computation of any EM property to determination of the EM field distribution just at the aperture openings. This is extremely efficient, especially for subwavelength apertures when convergence is reached quickly with the number of waveguide modes considered. In fact, in this regime, an accurate result for the transmittance can be achieved by considering only the fundamental waveguide mode in- (a)
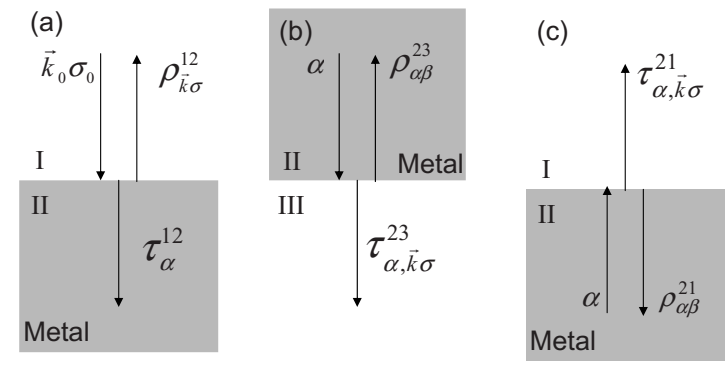

FIG. 3. Schematic definition of the different scattering coefficients for the EM fields at isolated interfaces dividing two semi-infinite media. (a) A plane wave impinging from medium I into medium II. (b) Incidence from a waveguide mode $\alpha$ in medium II into medium III. (c) As in (b) but now the waveguide mode $\alpha$ is backscattered in medium II or transmitted to medium I.

side each aperture. For the cases of either a single aperture or an infinitely periodic array, this single-mode approximation $(\alpha=0)$ allows a quasianalytical treatment of the set of equations (1) that now transforms into a set of just two linear equations:

$$
\begin{aligned}
& (G-\Sigma) E-G^{V} E^{\prime}=I, \\
& \left(G^{\prime}-\Sigma\right) E^{\prime}-G^{V} E=0,
\end{aligned}
$$

where $G \equiv G_{00}, G^{\prime} \equiv G_{00}^{\prime}, \Sigma \equiv \Sigma_{0}, G^{V} \equiv G_{0}^{V}$, and $I \equiv I_{0}$. The solution of this set of equations is

$$
E=\left(G^{\prime}-\Sigma\right) I / D, \quad E^{\prime}=G^{V} I / D,
$$

where the denominator $D$ is given by

$$
D=(G-\Sigma)\left(G^{\prime}-\Sigma\right)-\left(G^{V}\right)^{2} \text {. }
$$

An alternative way of finding the scattering coefficients, still within the coupled-mode framework, is by use of the multiple-scattering technique. Here the scattering coefficients of a stratified media are expressed in terms of the scattering coefficients of isolated interfaces. This is especially useful when surface EM modes are involved, as they show up in the properties of the corresponding interface. In all cases analyzed in this review, the structures are formed by three layers (reflection and transmission regions plus the perforated metal). Therefore, three different scattering problems involving two semi-infinite media must be solved (see Fig. 3).

In Fig. 3(a), the incident plane wave coming from the reflection region (medium I), characterized by $\vec{k}_{0} \sigma_{0}$ and unit amplitude, impinges on medium II (perforated metal). The incoming field is reflected back into the different diffraction modes in medium I with amplitude $\rho_{\vec{k} \sigma}^{12}$ and transmitted into object $\alpha$ of medium II with amplitude $\tau_{\alpha}^{12}$. The second two-media scattering problem is schematically shown in Fig. 3(b): a waveguide mode $\alpha$ coming from medium II impinges at the interface with medium III. It is reflected back at the interface into waveguide mode $\beta$ with amplitude $\rho_{\alpha \beta}^{23}$ and transmitted into the different plane waves of medium III, $\vec{k} \sigma$, with amplitudes $\tau_{\alpha, \vec{k} \sigma^{*}}^{23}$ The third scattering process is similar 


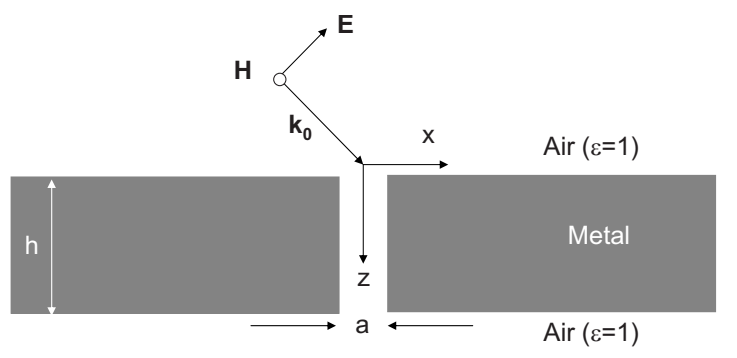

FIG. 4. Schematic of a single slit of width $a$ perforated on a metal film of thickness $h$ illuminated by $p$-polarized radiation. For the case of $s$-polarized incident radiation, vectors $\mathbf{E}$ and $\mathbf{H}$ are interchanged.

to the previous one, but with medium III and medium I exchanged [see Fig. 3(c)]. Detailed expressions for the two-media scattering coefficients can be found in the Appendix.

The total transmission process can be seen as an infinite sum of scattering processes involving the two interfaces plus light propagation inside medium II. The infinite series is geometric and can be summed up, yielding for the transmission coefficient $t_{\vec{k} \sigma}$ :

$$
t_{\vec{k} \sigma}=\sum_{\alpha, \beta, \gamma} \tau_{\alpha}^{12} e_{\alpha}\left(\delta_{\alpha \beta} \delta_{\alpha \gamma}-\rho_{\alpha \gamma}^{21} e_{\gamma} \rho_{\gamma \beta}^{23} e_{\beta}\right)^{-1} \tau_{\beta, \vec{k} \sigma}^{23},
$$

where $e_{\alpha} \equiv \exp \left(i q_{z \alpha} h\right)$, with $q_{z \alpha}$ the propagation constant associated with mode $\alpha$.

\section{TRANSMISSION THROUGH SINGLE APERTURES}

\section{A. One-dimensional aperture: Single slit}

We start our review of the transmission properties of subwavelength apertures by analyzing the simplest case: a single 1D slit of width $a$ perforated on a metal film of thickness $h$, illuminated by a plane wave with the wave vector lying in the $x-z$ plane (see Fig. 4). Since the system presents translational symmetry along the direction parallel to the slit, the analysis can be restricted to the perpendicular plane where the two light polarizations ( $s$ and $p$ ) are decoupled. For $p$-polarized light, the magnetic field is parallel to the slit ( $y$ direction) and the electric field has nonzero $x$ and $z$ components. For $s$-polarized light, the $E$ field is pointing to the $y$ direction and the $H$ field lies in the $x-z$ plane.

Most theoretical and experimental work has been focused on the optical response of a single slit under $p$-polarized illumination. On the theoretical side, there were several studies within the electrical engineering community during the 1970s and 1980s (Lehman, 1970; Hongo, 1971; Kashyap and Hamid, 1971; Neerhoff and Mur, 1973; Auckland and Harrington, 1978; Harrington and Auckland, 1980). They predicted the appearance of slit-cavity transmission resonances using different theoretical approaches (Fourier transforms, matrix techniques, and the method of moments). The interest in this type of 1D structure was renewed after theoretical studies found that the EOT phenomenon also emerges in 1D

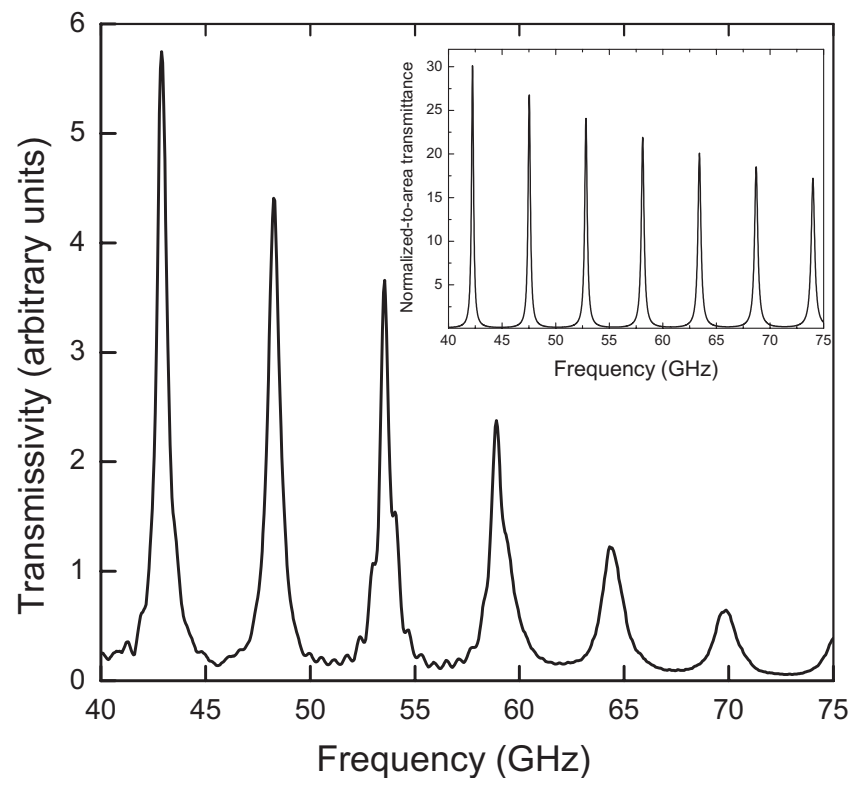

FIG. 5. Transmission (in arbitrary units) as a function of frequency for a single slit of width $75 \mu \mathrm{m}$ perforated on a metal film of thickness $28.2 \mathrm{~mm}$. Inset: Normalized-to-area transmittance calculated using the coupled-mode method. From Yang and Sambles, 2002.

periodic arrays of slits (Schroter and Heitmann, 1998; Porto et al., 1999). Takakura (2001) reconsidered the existence of transmission resonances in single isolated slits for $p$-polarized illumination, finding analytical expressions for the total transmittance at resonance. Yang and Sambles (2002) verified experimentally the existence of transmission resonances in the microwave regime (see Fig. 5).

In what follows, we analyze the transmission of $p$-polarized light through a single slit perforated on a PEC film by means of the coupled-mode method. In this case, the objects $\alpha$ in Eqs. (1) are the TM waveguide modes inside the slit (the TE modes can be neglected because they do not couple to the incident $p$-polarized radiation). The propagation constant associated with the TM mode $\alpha$ is $q_{z \alpha}=\sqrt{k_{\omega}^{2}-\pi^{2} \alpha^{2} / a^{2}}$ with $\alpha \geqslant 0$. Strictly speaking, mode $\alpha=0$ is a TEM mode (both the electric and magnetic fields are transverse) that does not present cutoff.

The inset of Fig. 5 shows the frequency dependence of the normalized-to-area transmittance (transmitted power normalized to that impinging directly at the slit opening), as obtained with the coupled-mode method (Bravo-Abad, Martin-Moreno, and Garcia-Vidal, 2004). The calculation includes as many TM modes as needed to achieve convergence. As in the experiment, a series of transmission peaks dominates the spectrum, with a peak height that decreases as the resonant frequency is increased. In order to gain physical insight, we consider the extreme subwavelength regime $a \ll \lambda$, in which a good approximation to the final transmittance spectrum can be obtained by considering only the TEM waveguide mode $(\alpha=0)$ inside the slit. We must then solve 
Eqs. (3) where, for this particular case, the coupling term $G=G^{\prime}$ is given by Eq. (A19). The spectral locations of the transmission peaks are associated with the resonant condition of the denominator for $E$ and $E^{\prime}$ in Eqs. (4), $|G-\Sigma|=\left|G^{V}\right|$. After some straightforward algebra, this condition can be rewritten as

$$
\tan k_{\omega} h=2 \operatorname{Re} G /|G|^{2}-1 .
$$

In the limit of extremely narrow slits $(G \rightarrow 0)$, this last equation predicts the appearance of transmission peaks close to the Fabry-Perot resonance condition $\sin k_{\omega} h$ $=0$. This shows that cavity resonances inside the single slit are responsible for the emergence of transmission peaks for $p$-polarized light. A general expression for the normalized-to-area transmittance at resonance $T_{\text {res }}$ can be obtained using the resonant condition $|G-\Sigma|=\left|G^{V}\right|$ in the equation $T=\operatorname{Re}\left(G^{V} E^{*} E^{\prime}\right)$ :

$$
T_{\text {res }}=|I|^{2} / 4 \operatorname{Im}(G) \text {. }
$$

An accurate estimation for $T_{\text {res }}$ can then be obtained from Eq. (8) by taking the limit $a \ll \lambda$ in the expressions for $\operatorname{Im}(G)$ given in Eq. (A19), leading to $T_{\text {res }}^{\text {slit }}=\lambda / \pi a$. This analytical result predicts a linear increase of $T_{\text {res }}$ with the resonant wavelength, as observed in Fig. 5. The analytical expression for $T_{\text {res }}$ was first derived by Harrington and Auckland (1980) using a circuit model and is strictly valid within the PEC approximation. Experiments performed by Suckling et al. (2004) showed that, even at microwave frequencies, this single linear dependence of $T_{\text {res }}$ vs $\lambda / a$ does not hold for very narrow slits because of the finite conductivity of the metal. Nevertheless, in recent experimental work in the terahertz regime, Seo et al. (2009) showed that the transmittance and the corresponding $E$-field enhancement increase monotonically as a function of $\lambda / a$ for slits as narrow as $20 \mathrm{~nm}$.

An interesting feature of these localized Fabry-Perot resonances is that they are quite insensitive to the angle of incidence when the plane of incidence is perpendicular to the slit width (Bravo-Abad, Martin-Moreno, and Garcia-Vidal, 2004). The case in which the plane of incidence is parallel to the slit has also been analyzed (Suckling et al., 2005; Gordon, 2007). Here the spectral locations of the resonant modes strongly depend on the angle of incidence, leading to a much richer behavior in the transmission spectrum.

As an intermediate case between a single slit and a periodic array of slits, the transmission of $p$-polarized radiation through two slits has also been analyzed (Schouten et al., 2005), revisiting the famous Young double-slit experiments. By studying the dependence of the transmission spectra with the distance between the slits, they observed that the SPPs launched by the two slits could interfere (constructively or destructively), enhancing or reducing the intensity of the far-field pattern. This interference picture was further corroborated by more sophisticated theoretical calculations (Lalanne, Hugonin, and Rodier, 2005).

A coupled-mode analysis can also be done when a single slit is illuminated by $s$-polarized light. Now, only

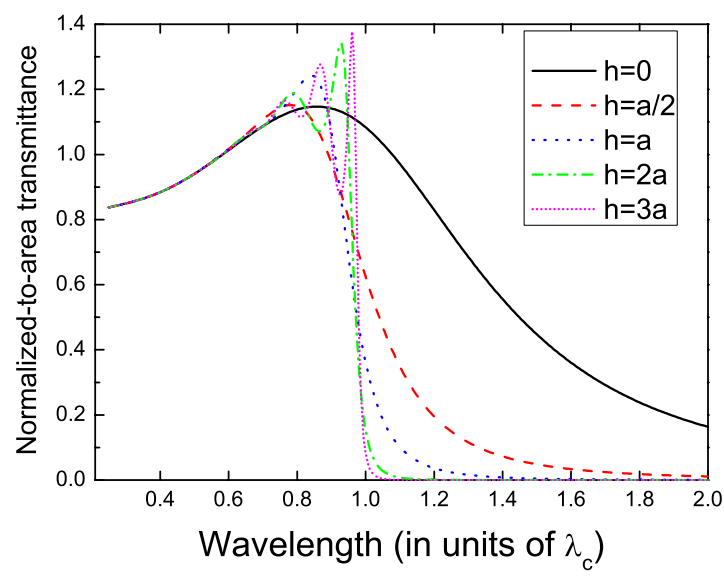

FIG. 6. (Color online) Normalized-to-area transmittance vs wavelength (in units of the cutoff wavelength of the fundamental TE mode $\lambda_{C}=2 a$ ) through a single slit of width $a$ perforated on a PEC film of different thicknesses $h$, ranging from $h=0$ to $3 a$. The normally incident plane wave is $s$ polarized.

the TE modes inside the slit need to be considered. Unlike TM modes, these TE waveguide modes present cutoff. The electromagnetic coupling between TE modes is governed by $G_{\alpha \beta}$ terms that, due to the different boundary conditions, are different from those of the $p$-polarized case. The expression in real space of the $s$-polarization propagator $\hat{G}$ can be found in the Appendix [see Eq. (A21)]. In Fig. 6 we show the normalizedto-area transmittance spectra for slits with the same width $a$ but different thicknesses. Note that, in contrast to the $p$-polarized case, the transmission process is dominated by the existence of a cutoff wavelength for TE modes, $\lambda_{C}=2 a$. For $\lambda>\lambda_{C}$ and thick enough films, the transmission is strongly attenuated due to the evanescent character of the EM fields inside the slit. FabryPerot resonances similar to those found for $p$-polarized light appear in the spectrum for $\lambda<\lambda_{C}$.

The case of transmission of $s$-polarized plane waves through single slits perforated in real metals (silver and aluminum films) at optical frequencies has also been analyzed theoretically (Schouten et al., 2003). In a real metal the transmissivity can be much higher than for the PEC case due to a reduction of the cutoff wavelength. Accompanying this boost in transmission, they discovered that phase singularities in the field of power flow may emerge near the slit.

\section{B. Two-dimensional single apertures}

\section{Circular holes}

Although the curiosity and technological interest in the phenomenon of transmission of light through small holes in an opaque screen started some centuries ago, the first accurate treatment of the electromagnetic coupling through a very small hole was presented by Bethe (1944) in the idealized case of a zero-thickness perfect conductor film perforated with a circular hole of radius $r$. In his calculation, the screen and the aperture were 


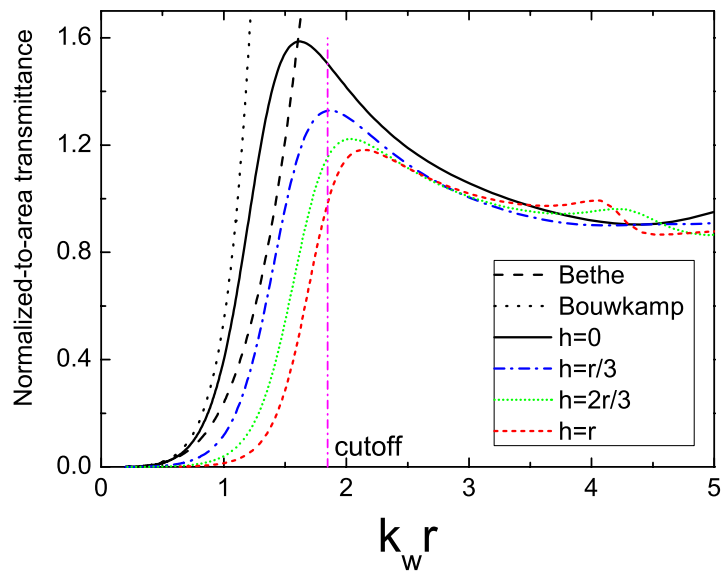

FIG. 7. (Color online) Normalized-to-area transmittance for a normally incident plane wave vs $k_{\omega} r$ for a circular hole of radius $r$ perforated on PEC films of different thicknesses $h$. Dashed and dotted lines show the predictions of Eqs. (9) and (10), respectively. The cutoff condition of the hole waveguide, $k_{\omega} r=1.85$, is also depicted.

replaced by two emitting electric and magnetic dipoles. Bethe derived a simple expression for the normalizedto-area transmittance in the extreme subwavelength limit $\left(k_{\omega} r \ll 1\right)$ :

$$
T_{\mathrm{Be}}=64\left(k_{\omega} r\right)^{4} / 27 \pi^{2} \text {. }
$$

This equation implies that $T_{\mathrm{Be}}$ scales as $(r / \lambda)^{4}$, predicting a rapid drop of the optical transmission as $\lambda$ is increased. Later Bouwkamp (1950) found that the transmittance could be written as a series in $k_{\omega} r$ in which the expression obtained by Bethe was just the first term. Bouwkamp derived the following expression for the normalized-to-area transmittance $T_{\mathrm{Bo}}$ :

$$
T_{\mathrm{Bo}}=T_{\mathrm{Be}}\left(1+\frac{22}{25}\left(k_{\omega} r\right)^{2}+\frac{7312}{18375}\left(k_{\omega} r\right)^{4}+O\left(k_{\omega} r\right)^{6}\right) .
$$

Notice that this series seems to diverge if $\lambda \leqslant 2 \pi r$. Therefore, the expressions given by Bethe and Bouwkamp have limited value for the EOT phenomenon, in which the resonant wavelength is typically two to three times the diameter of the holes. Furthermore, $T_{\mathrm{Be}}$ and $T_{\mathrm{Bo}}$ were obtained for an opaque screen with negligible thickness. A phenomenological extension of the BetheBouwkamp theory to finite thickness and larger apertures was presented by Cohn (1952). He showed that, because of the evanescent character of the EM files inside the aperture, the transmission efficiency is further attenuated for wavelengths longer than the cutoff wavelength of the hole, which for a circular hole is $\lambda_{C}=3.4 r$. Roberts (1987) presented the first rigorous calculation of the transmittance spectrum of a single circular hole perforated on a PEC film of finite thickness. Figure 7 shows the normalized-to-area transmittance spectra obtained with the coupled-mode method, which for an isolated circular hole in a PEC is equivalent to the method used by Roberts. As seen in the figure, the expressions of
Bethe and Bouwkamp [Eqs. (9) and (10)] are reasonable approximations in the extreme subwavelength limit $\left(k_{\omega} r \ll 1\right)$ for $h=0$ but overestimate the total transmission for $h \neq 0$ in all ranges of $k_{\omega} r$. Recently, analytical expressions for the transmittance through single subwavelength holes (circular and rectangular) perforated in PEC films of finite thickness have been provided by Nikitin, Zueco, et al. (2008).

The invention of the scanning near-field optical microscope (SNOM) in the 1980s (Lewis et al., 1984; Pohl et al., 1984), and the discovery of the EOT phenomenon (Ebbesen et al., 1998) stimulated new experimental and theoretical studies on both the total intensity and the spatial distribution of the light transmitted through a small circular hole. The first accurate numerical calculation of the transmittance through a circular hole perforated in a real metal at optical frequencies was reported by Wannemacher (2001). Using the MMP method, Wannemacher was able to predict the appearance of transmission resonances in very thin silver films $(50-150 \mathrm{~nm})$, which he ascribed to SPP excitation.

The EM fields emerging from single circular subwavelength apertures have been experimentally mapped by raster scanning the aperture of a near-field microscope over a molecule (Betzig and Chichester, 1993; Veerman et al., 1999; van Hulst et al., 2000; Sick et al., 2001; Moerland et al., 2005). Through their dipole nature, single molecules act as subwavelength detectors of the $E$ field behind the aperture. The molecular fluorescence yields direct insight into the local EM fields: the molecule's fluorescence intensity $I_{\mathrm{fl}}$ is related to the local electrical field $\vec{E}$ as $I_{\mathrm{fl}} \propto|\vec{\mu} \cdot \vec{E}|^{2}$, where $\vec{\mu}$ is the transition dipole of the molecule. As a result, the fluorescence is not only a measure of the magnitude of the electric field but is also sensitive to its direction with respect to the dipole.

Experiments have also revealed that the angular distribution of the transmitted light is far from being uniform (Obermuller and Karrai, 1995; Degiron et al., 2004), making the measurement of the total transmittance through an isolated hole a delicate task. This type of measurement was presented by Degiron et al. (2004) for circular holes milled in suspended silver films of various thicknesses. The reported total transmission spectra are shown in Fig. 8, demonstrating the presence of a transmission resonance with an intensity that decreases rapidly with increasing $h$. The calculated cutoff wavelength for the considered holes is $\sim 600 \mathrm{~nm}$ (Moreno, 2008); this value is larger than it would be if the hole were in a PEC $(460 \mathrm{~nm})$ due to non-negligible skin depth of silver in this frequency regime. This cutoff is consistent with the decay of the transmittance at large $h$ but the observed transmission peak appears at a wavelength longer than the cutoff wavelength. Based on measurements of the spatial and spectral characteristics of the light emission induced by a high-energy electron beam, Degiron et al. (2004) concluded that this resonance stems from the excitation of a localized surface plasmon at the edges of the hole. In a different experi- 


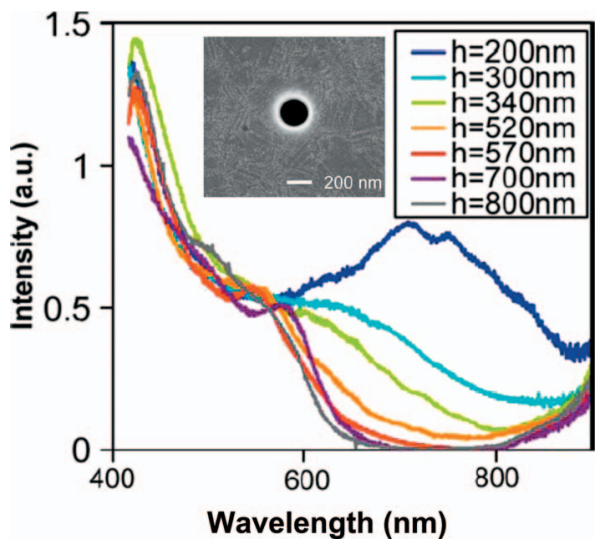

FIG. 8. (Color) Transmission spectra for single circular holes. Inset: Scanning electron micrograph (SEM) image of a single circular hole of radius $r=135 \mathrm{~nm}$ perforated on a suspended silver film. Main panel: Transmittance spectra for normal incident radiation for a range of film thicknesses $h$. Each curve is an average of spectra obtained for several isolated holes of the same size. From Degiron et al., 2004.

mental study, Prikulis et al. (2004) showed that both scattering and extinction spectra of optically thin gold films perforated by subwavelength circular holes exhibit an optical resonance in the visible range. They also assigned the resonance to a dipolar localized surface plasmon at the hole circumference.

A different physical explanation for this transmission resonance appearing in circular holes has been reported recently (Park et al., 2008). Using a plasmon hybridization approach, they argued that, when the hole is present, extended SPPs of certain wavelengths induce a large dipole moment across the hole in such a way that the hole mediates a resonant coupling between these specific SPPs and the incident plane wave.

Several theoretical investigations (Chang et al., 2005; Popov et al., 2005) have corroborated the localized nature of those transmission resonances, as suggested in the original experiments (Degiron et al., 2004). In particular, Popov et al. (2005) concluded that there are two main contributions to the transmitted EM field: localized surface plasmon excitation produced by the ridges of the hole with an electric field that is almost normal to that of the incident wave, and a much weaker radiation from the electric dipole formed by the charge accumulated at the same points. FDTD calculations (Chang et al., 2005) indicated that the spectral position of the transmission maximum redshifts with increasing hole diameter and blueshifts with increasing film thickness.

Another set of theoretical works (Catrysse et al., 2005; Webb and $\mathrm{Li}, 2006$ ) has investigated the existence of propagating waveguide modes in a cylindrical hole for arbitrarily small diameters. These works demonstrated that the cutoff wavelength of the hole waveguide (perforated on a metal characterized by a Drude dielectric function) does not approach zero when the hole radius tends to zero (as it occurs in a PEC) but to the value $\sqrt{2} \lambda_{p}$, with $\lambda_{p}$ the wavelength of the bulk plasmon. Note,

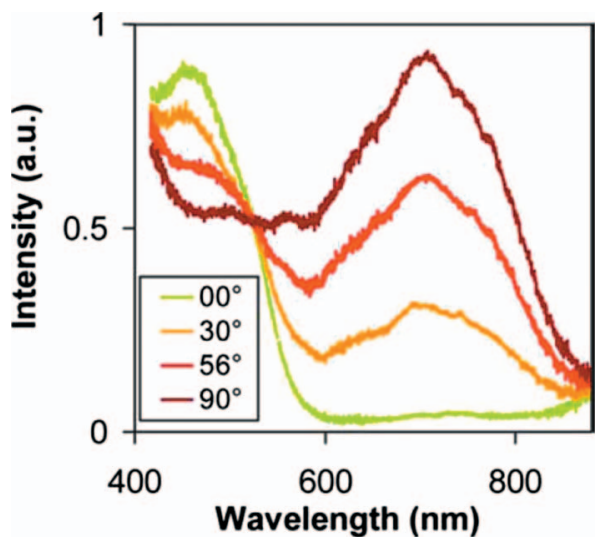

FIG. 9. (Color) Normal incidence transmission spectra of an isolated rectangular aperture for various linear polarizations, defined as the angle that forms the $E$ field with the long side of the rectangle. The thickness of the free-standing silver film is $h=700 \mathrm{~nm}$ and the sides of the rectangle are $a_{x}=210 \mathrm{~nm}$ and $a_{y}=310 \mathrm{~nm}$. From Degiron et al., 2004.

however, that for metals this plasmon wavelength is in the ultraviolet regime.

Recently, using terahertz-light excitation, Adam et al. (2008) measured the time and frequency-dependent $E$ field in the vicinity of subwavelength circular holes (perforated in a 200-nm-thick gold film deposited on a GaP substrate), with subwavelength spatial and subcycle temporal resolution. As expected from the calculations of Bouwkamp (1950) for a circular hole perforated through a PEC film, the $E$ field is highly concentrated near the edges of the hole. Interestingly, they also reported the emergence of a transmission resonance that is spectrally located close to the cutoff frequency of the lowest-order mode, the $\mathrm{TE}_{11}$ mode, calculated as if the hole were filled with a material whose refractive index coincides with that of the substrate. Further theoretical investigations are needed in order to reveal the physics behind this resonance and its relation to the cutoff transmission

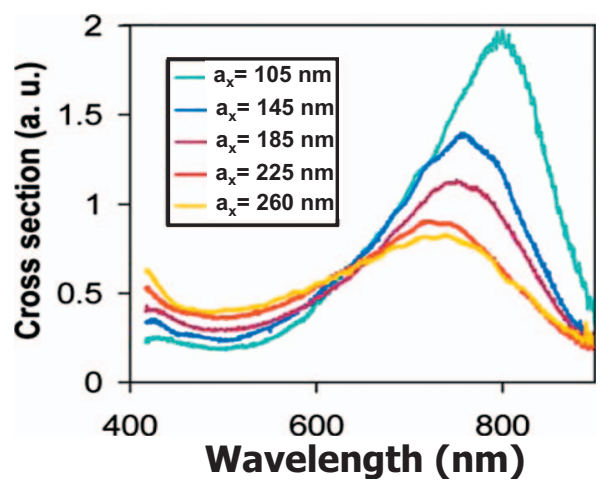

FIG. 10. (Color) Transmission spectra of isolated rectangular apertures with fixed $h=300 \mathrm{~nm}$ and $a_{y}=270 \mathrm{~nm}$ with various $a_{x}$ $\left(105<a_{x}<260 \mathrm{~nm}\right)$, perforated through silver films. All structures are illuminated by a normal incident plane wave whose $E$ field points along the short side of the rectangle. From Degiron et al., 2004. 


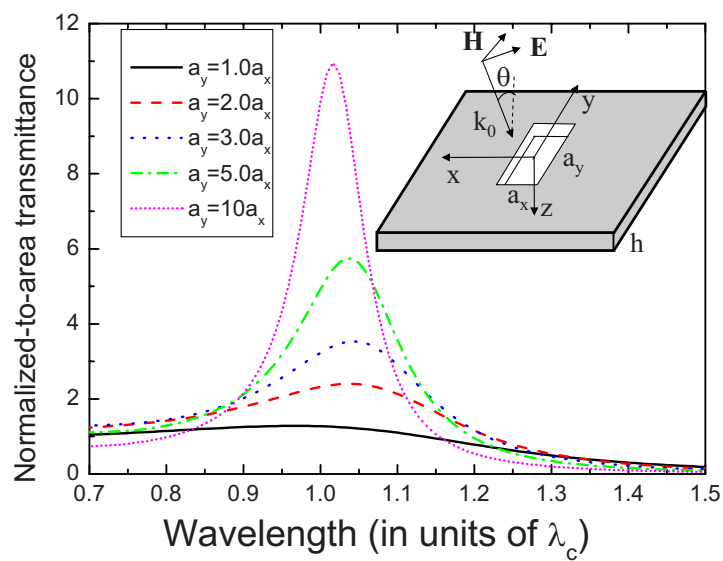

FIG. 11. (Color online) Transmission through a single rectangular hole. Inset: Schematic of a single rectangular hole of sides $a_{x}$ and $a_{y}$ perforated through a metallic film of thickness $h$. The structure is illuminated by a $p$-polarized plane wave. Main panel: Normalized-to-area transmittance vs wavelength (in units of the cutoff wavelength $\lambda_{C}=2 a_{y}$ ) for a normally incident $p$-polarized plane wave impinging on single rectangular holes for different ratios $a_{y} / a_{x}$. The thickness of the metal film is $h=a_{y} / 3$. From Garcia-Vidal, Moreno, et al., 2005.

resonances appearing for rectangular and annular holes, as described in Sec. II.B.2.

Apart from their transmission properties, isolated circular holes have also been studied as nanometric generators of SPPs (Sonnichsen et al., 2000; Yin et al., 2004; Chang et al., 2005; Baudrion et al., 2008). The holes act as pointlike localized sources of SPPs and the coupling efficiency can be made as large as 20\% (Baudrion et al., 2008) by a proper design of the geometrical parameters.

\section{Rectangular holes}

Given the large difference in the optical response of slits under $s$ - or $p$-polarized illumination, rectangles can be expected to present strong polarization effects. The experimental study of the optical transmission properties of a single rectangular hole was carried out by Degiron et al. (2004). As shown in Fig. 9, two distinct peaks can be observed in the transmission spectra by simply changing the polarization of the incident field with respect to the long axis of the rectangle. Degiron et al. (2004) also analyzed the dependence of the transmission resonances with the aspect ratio of the rectangular hole. The experiments showed that the peak height increases and its spectral location shifts to larger wavelengths as the ratio between the two sides of the rectangle $a_{y} / a_{x}$ is enlarged (see Fig. 10).

In this section we also analyze theoretically the properties of a single rectangular hole of sides $a_{x}$ and $a_{y}$ perforated on a metallic film of thickness $h$ (see inset of Fig. 11) using the coupled-mode method. In the first part of this analysis the metal is treated as a PEC (Garcia-Vidal, Moreno, et al., 2005). Then, the effect of a finite dielectric constant on the resonant transmission properties of a single rectangular hole will be addressed (GarciaVidal, Martin-Moreno, et al., 2006). We focus on the case of $p$-polarized incident radiation, with the in-plane $E$-field component pointing along the $x$ direction (short side of the rectangle).

Figure 11 depicts the calculated normalized-to-area transmission spectra for rectangular holes in which the aspect ratio $\left(a_{y} / a_{x}\right)$ is varied between 1 (square hole) and 10. The thickness of the PEC film is fixed in all cases at $h=a_{y} / 3$. As shown below, this is not an essential parameter regarding the emergence of transmission resonances. As the ratio $a_{y} / a_{x}$ is increased, a transmission peak develops close to the cutoff wavelength $\left(\lambda_{C}=2 a_{y}\right)$, with increasing maximum transmittance and decreasing linewidth. In all cases, above cutoff the transmittance decreases strongly with wavelength due to both the evanescent decay of the EM fields inside the hole and the poor coupling of the incident wave with the waveguide modes in this limit. The presence of a transmission resonance at $\lambda=2 a_{y}$ for a thin PEC film can be understood by taking advantage of Babinet's principle. In this limit, the complementary system of a rectangular hole illuminated by $p$-polarized light is a PEC rectangular stripe of length $a_{y}$ in vacuum excited by an electric field pointing along the long side of the rectangle whose dipolar resonance appears precisely at $\lambda \approx 2 a_{y}$.

As in the case of a single slit, a good approximation to the final result can be achieved by considering only the first TE waveguide mode inside the hole. This mode has a propagation constant $q_{z}=\sqrt{k_{\omega}^{2}-\left(\pi / a_{y}\right)^{2}}$ and associated admittance $Y_{\mathrm{TE}}=q_{z} / k_{\omega}$. Within the single-mode approximation, the set of equations (3) also applies for this case. Normalizing the incident $p$-polarized plane wave such that the incoming energy flux over the hole area is unity, we obtain for the illumination term,

$$
I=\frac{4 i \sqrt{2}}{\pi} \frac{\operatorname{sinc}\left(k_{\omega} a_{x} \sin \theta / 2\right)}{\sqrt{\cos \theta}},
$$

where $\theta$ is the angle of incidence. The expression for the self-illumination of the fundamental TE mode via vacuum modes $G$ can be found in the Appendix [see Eq. (A22)]. The spectral locations of the transmission resonances are given by the condition $|G-\Sigma|=\left|G^{V}\right|$, leading to

$$
\tan \left(q_{z} h\right)=2 Y_{\mathrm{TE}} \operatorname{Re}(G) /\left(|G|^{2}-Y_{\mathrm{TE}}^{2}\right) .
$$

Due to the coupling to free-space modes, these resonances are shifted from those predicted from the FabryPerot condition $\tan \left(q_{z} h\right)=0$, which would arise if the optical path inside the hole only were taken into account. Equation (12) is satisfied at cutoff when $q_{z}=Y_{\mathrm{TE}}=0$. Therefore, the physical origin of the transmission resonances appearing for rectangular holes at $\lambda \approx \lambda_{C}$ stems from the excitation of a Fabry-Perot resonance in which the propagation constant is zero.

Within the single-mode approximation, the only dependence of the transmittance on the angle of incidence stems from the illumination term $I$. Then the location of the resonant peaks observed in Fig. 11 does not depend on $\theta$. Moreover, in the extreme subwavelength limit 
$\left(\lambda \gg a_{x}\right)$, the term sinc in $I$ [see Eq. (11)] approaches 1, yielding a simple $1 / \cos \theta$ dependence for the peak heights in the normalized-to-area transmittance spectra.

An accurate analytical approximation for the transmittance at resonance $T_{\text {res }}$ can be obtained by making use of the general expression for $T_{\text {res }}$ given by Eq. (8). In the extreme subwavelength regime and using Eq. (A22), we obtain $\operatorname{Im}(G) \approx 32 a_{x} a_{y} / 3 \pi \lambda^{2}$, leading to

$$
T_{\text {res }} \approx \frac{3}{4 \pi} \frac{\lambda_{\text {res }}^{2}}{a_{x} a_{y}} .
$$

This expression predicts that for the transmission peak located near the cutoff wavelength $\lambda_{\text {res }} \approx 2 a_{y}, T_{\text {res }}$ $\approx(3 / \pi) a_{y} / a_{x}$, explaining the numerical results shown in Fig. 11, in which an almost linear increase of the peak height versus ratio $a_{y} / a_{x}$ is observed.

Additionally, even for a fixed aspect ratio $a_{y} / a_{x}$, Eq. (13) gives us a clue for further enhancing the transmission: namely, by filling the hole with a material with dielectric constant $\epsilon>1$, as this increases the cutoff wavelength. Now, the propagation constant of the fundamental TE mode is $q_{z}=\sqrt{\epsilon k_{\omega}^{2}-\left(\pi / a_{y}\right)^{2}}$ and its cutoff wavelength is $\lambda_{C}=2 \sqrt{\epsilon} a_{y}$. According to Eq. (13), this should lead to a linear increase of $T_{\text {res }}$ with $\epsilon$. Note that this increase of the transmission when the hole is filled with material with $\epsilon>1$ also occurs for the case of circular or square holes (De Abajo, 2002). Remarkably, in rectangular holes, this mechanism acts almost independently of the "geometric" enhancement appearing at large aspect ratios, so $T_{\text {res }}$ is proportional to both $a_{y} / a_{x}$ and $\epsilon$.

Associated with this resonant phenomenon, there is an enhancement of the EM fields. A direct evaluation of $|E|^{2}=\left|E^{\prime}\right|^{2}$ at the resonant condition yields

$$
|E|_{\text {res }}^{2}=\left|E^{\prime}\right|_{\text {res }}^{2}=|I|^{2} / 4[\operatorname{Im}(G)]^{2}
$$

leading to an enhancement of the intensity of the $E$ field at the hole (with respect to the incident one) that scales with $\lambda_{\text {res }}$ as $\lambda_{\text {res }}^{4} /\left(a_{y} a_{x}\right)^{2}$, much larger than the enhancement in the transmittance. This implies that, in the process of resonant transmission, light is highly concentrated on the entrance and exit sides of the hole and only a small fraction of this light is finally transmitted into the far field. Still, as we have seen the amount of light that is finally transmitted is larger than the one directly impinging in the hole.

Experimental verification of the existence of cutoff transmission resonances in rectangular holes was recently reported by Lee et al. (2007) in the terahertz regime of the EM spectrum. They considered sets of rectangular holes where all holes had the same dimensions and orientation but were randomly positioned. The measured transmission spectra confirmed the existence of resonances associated with the cutoff. In Fig. 12, three representative transmission spectra are displayed that show the predicted increase in the transmittance as the aspect ratio of the rectangular hole is increased.

It is clear that the transmission resonances appearing for a rectangular hole perforated on a PEC film have

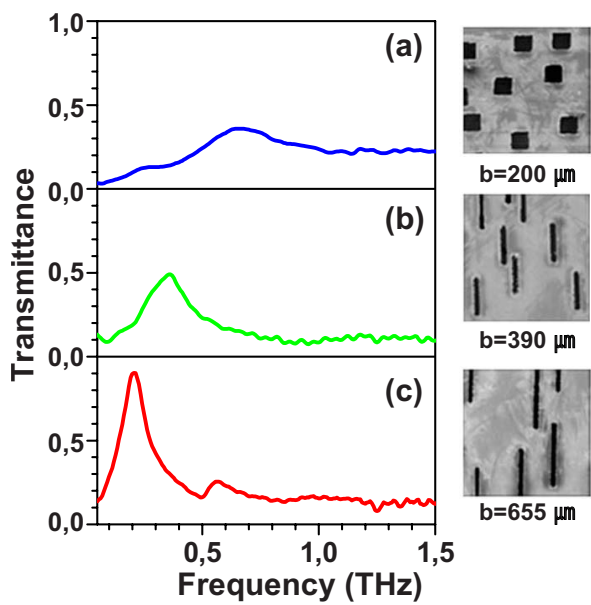

FIG. 12. (Color online) Normalized transmission amplitudes, at normal incidence, of random arrays of holes with dimensions (a) $a_{x}=a_{y}=200 \mu \mathrm{m}$, (b) $a_{x}=70 \mu \mathrm{m}$ and $a_{y}=390 \mu \mathrm{m}$, and (c) $a_{x}=70 \mu \mathrm{m}$ and $a_{y}=655 \mu \mathrm{m}$. In all three cases the metal thickness is $h=17 \mu \mathrm{m}$. Insets are scanning electronic microscope (SEM) images of the 2D random arrays. From Lee et al., 2007.

strong similarities with those found experimentally by Degiron et al. (2004) in the optical regime, in particular, the experimentally observed increase in the transmittance at resonance with increasing aspect ratio. Simulations based on the coupled-mode method with the inclusion of SIBCs presented a good agreement with the experimental data and a close correspondence between the spectral locations of the transmission peak with the cutoff wavelength of the hole waveguide perforated on a real metal (Garcia-Vidal, Martin-Moreno, et al., 2006). This cutoff wavelength is redshifted with respect to its PEC value due to the penetration of the EM fields inside the metal at optical frequencies (Gordon and Brolo, 2005).

\section{TRANSMISSION THROUGH ARRAYS OF APERTURES}

\section{A. One-dimensional periodic arrays of slits}

\section{Theoretical results}

Before the discovery of the EOT phenomenon in periodic arrays of subwavelength holes (Ebbesen et al., 1998), there had been only a few theoretical and experimental works on the resonant transmission features of the so-called transmission gratings, i.e., periodic arrays of 1D slits perforated through metallic films (see Fig. 13). After more than ten years of EOT research, there are many more theoretical studies devoted to analyzing the emergence of the EOT phenomenon in 1D arrays of subwavelength slits for $p$-polarized light (no transmission resonances appear for $s$-polarized radiation) than experimental ones. This is because the $1 \mathrm{D}$ geometry showing EOT behavior is the easiest one to treat theoretically. Schroter and Heitmann (1998) presented the first numerical study in the optical regime, finding that, as in the case of the experiments for $2 \mathrm{D}$ hole arrays, the 


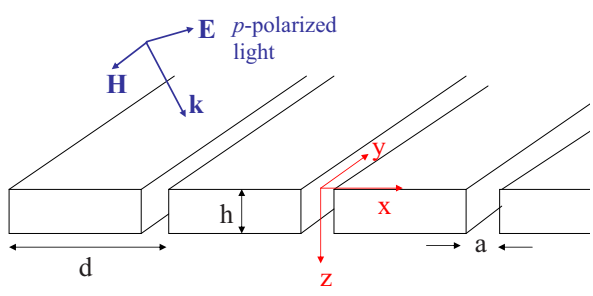

FIG. 13. (Color online) Schematic of a 1D periodic array of slits (period $d$, width of the slits $a$ ) perforated on a metallic film of thickness $h$. The structure is illuminated by $p$-polarized radiation, with the $E$ field lying in the $x-z$ plane.

transmission spectrum presented peaks whose spectral locations indicated that SPPs were involved in the resonant transmission process.

Later Porto et al. (1999) demonstrated that there are two possible ways of transferring $p$-polarized light efficiently from the upper surface to the lower one: by the coupling of the incident plane wave with cavity resonances located inside the slits (similar to those found in the single slit case described in Sec. II.A) or by the excitation of coupled surface EM modes on both surfaces of the grating, appearing when the slits form a periodic array. The existence of these two channels for enhancing the transmittance has been corroborated by many subsequent theoretical studies (Astilean et al., 2000; Lalanne et al., 2000; Collin et al., 2001, 2002; Garcia-Vidal and Martin-Moreno, 2002; Liu and Tsai, 2002; Hooper and Sambles, 2003; Lee and Park, 2005; Marquier et al., 2005). Different names have been used to refer to them: horizontal and vertical resonances, surface and cavity resonances, etc. The two resonant mechanisms are clearly differentiated only for some ranges of the geometrical parameters. In general, due to the existence of a propagating TEM mode inside the slits which always couples both interfaces, the two cited mechanisms are mixed in a way that depends strongly on the geometrical parameters of the structure (Collin et al., 2001, 2002; Marquier et al., 2005; Catrysse et al., 2006). Marquier et al. (2005) characterized this hybridization between cavity modes and surface EM modes in the transmission resonances. Figure 14 presents their theoretical results for a free-standing silver film. Figure 14(a) shows the transmission versus both frequency and parallel momentum, while Fig. 14(b) renders the dispersion relation of the mode responsible for the enhanced transmission (branch $B-C$ ). Figure 14(c) shows the surface-cavity (SC) ratio as a function of parallel momentum. This SC ratio is calculated by comparing the EM energies associated with the cavity and SPP components of the guided mode. This is done quantitatively by integration the of EM energy within the slit (cavity component) and on both interfaces (SPP component), respectively. As shown in Fig. 14, for $k_{x}=0$, the character of the mode is mainly cavitylike ( $75 \%$ for the geometrical parameters considered in this study). As the parallel momentum is increased, the character is evolving to be SPP-like.

Some have criticized the role of SPPs in the enhanced transmission process (Cao and Lalanne, 2002; Crouse
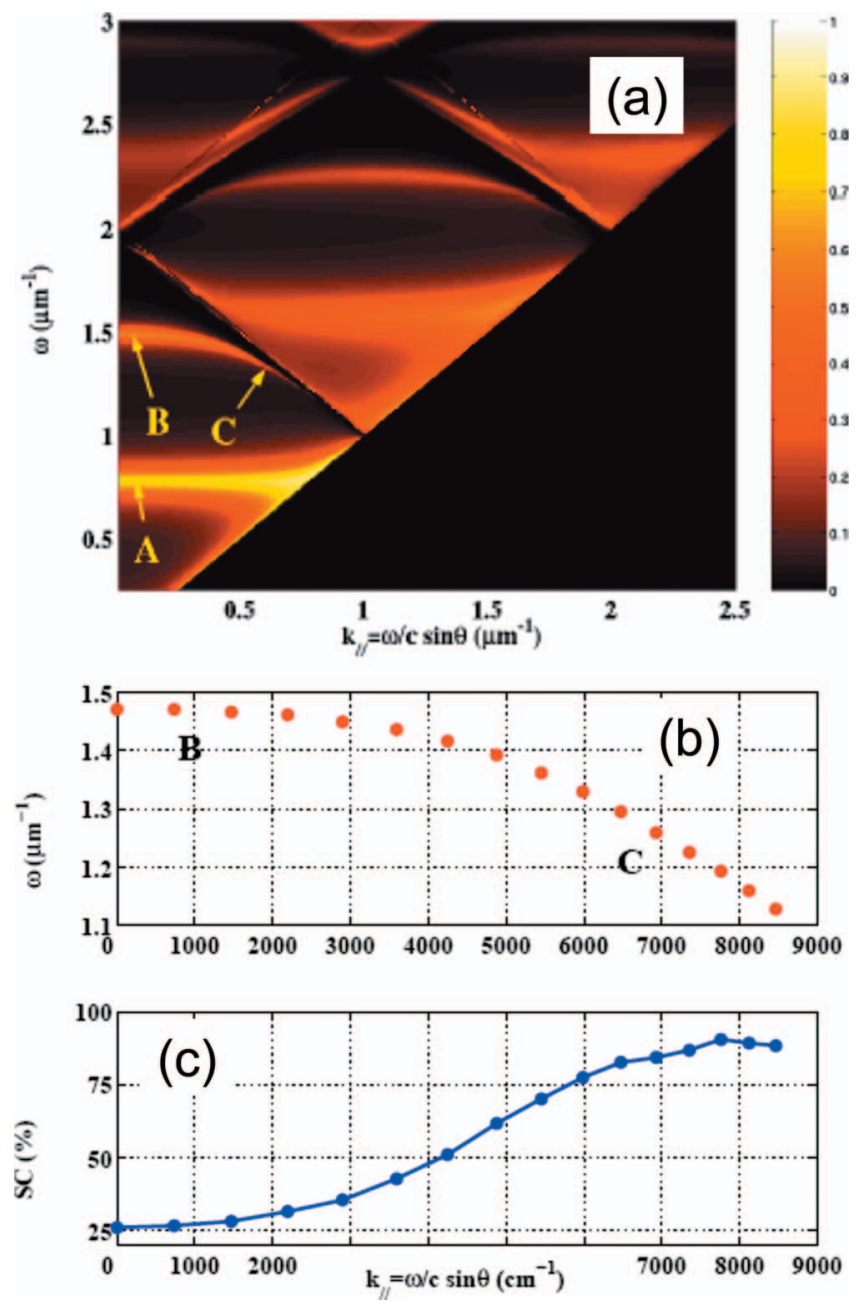

FIG. 14. (Color) Transmission resonances in 1D transmission gratings. (a) Transmission efficiency as a function of both $\omega$ and $k_{x}$. Period of the grating, $d=500 \mathrm{~nm}$; width of the slits, $a$ $=50 \mathrm{~nm}$; and thickness $h=400 \mathrm{~nm}$. (b) Branch $B-C$ of the dispersion relation. (c) Surface-cavity (SC) ratio for the transmission branch displayed in (b). From Marquier et al., 2005.

and Keshavareddy, 2005; Xie et al., 2005). Based on the existence of a transmission minimum appearing at the spectral location of the SPP in a flat, uncorrugated metal surface, they argued that the excitation of SPPs is in fact detrimental for the transmission. We show in Sec. III.A.2 that the dispersion relation of the SPPs changes when the metal film is perforated with a 1D array of slits and, indeed, the transmission peaks emerge at the spectral locations of the SPPs for the corrugated surface. As stressed by Treacy $(1999,2002)$, SPPs are an intrinsic component of the diffracted EM field in a real metal but the analysis of the transmission anomalies requires considering the full diffracted EM field. In other words, the coupling between indentations is not necessarily only through the SPP modes of the flat surface, a point elaborated in Sec. III.B. However, regardless of the precise form of the coupling, EM coupled arrays of indentations in a metal film can present bound modes. This occurs even if the metal is a PEC, where the EM coupling between indentations is purely through diffraction modes 


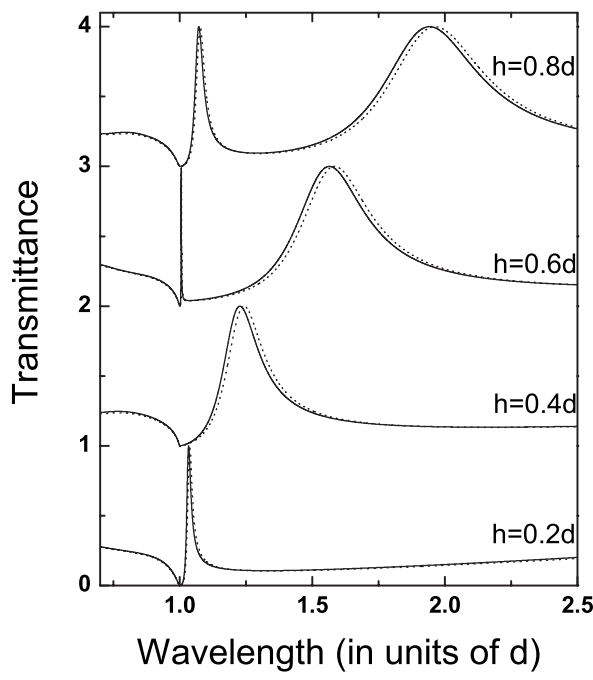

FIG. 15. Total transmittance (normalized to the unit cell of the array) of a normal-incidence $p$-polarized plane wave vs wavelength (in units of $d$ ) for periodic arrays of slits of fixed width, $a=0.2 d$, and several thicknesses $h=0.2 d, 0.4 d, 0.6 d$, and $0.8 d$. Full lines show the calculations in which the ten lowest TM waveguide modes are considered whereas dotted lines render the results when only the first waveguide mode (TEM $\alpha=0$ ) is included.

and does not contain contributions from SPPs (Pendry et al., 2004; Garcia-Vidal, Martin-Moreno, and Pendry, 2005).

\section{Coupled-mode analysis}

In what follows we analyze theoretically the transmission resonances appearing in a periodic array of $1 \mathrm{D}$ slits illuminated by $p$-polarized radiation using the coupledmode method. We first consider the case of a perforated PEC film (in which no SPPs can be excited and there is no absorption). In the second part of this study, we show how this PEC picture is slightly modified when a real metal in the optical regime (and the associated SPPs) is considered.

As in previous cases, the system of equations (1) can be used to describe the transmission properties of the structure depicted in Fig. 13. Now the objects $\alpha$ are just Bloch combinations of TM waveguide modes inside the slits. As we are dealing with an infinitely periodic array, the coupling term between waveguide modes $\alpha$ and $\beta$, $G_{\alpha \beta}$, whose general expression is given by in Eq. (2), can now be written as a discrete sum over $p$-polarized diffracted modes.

In Fig. 15 we show the transmittance spectra for a 1D array of slits of period $d$, width $a=0.2 d$, and different values of $h$. Note that, as the PEC approximation is considered, $d$ can be used as a unit length of the structure. The system is illuminated by a normally incident $p$-polarized plane wave and the modal expansion of the EM fields inside the slits contains ten TM modes (full lines). A series of transmission peaks (reaching 100\% transmission) dominates the spectrum. Two types of transmission resonances can be distinguished: very nar- row transmission peaks appearing at a wavelength close to $d$ (with strong $E$-fields at the horizontal surfaces) and wider peaks emerging at longer wavelengths (related to Fabry-Perot resonances, with strong $E$ fields inside the slits). In fact, as a function of the metal thickness there is a continuous shift of the resonances and, accordingly, a continuous modification of their character, as discussed before. An interesting point is that the transmission peak emerging close to $d$ also appears in a perforated PEC film which, in principle, does not support SPPs. As shown later, the physical origin of this resonant feature stems from the excitation of geometrically induced surface EM modes that mimic the behavior of SPPs in perforated PEC structures.

A quasianalytical treatment of this problem can be carried out (Garcia-Vidal and Martin-Moreno, 2002) because consideration of only the TEM mode $(\alpha=0)$ inside the slits already leads to accurate results for the transmittance (see dotted lines in Fig. 15). Within the singlemode approximation, the zero-order transmission coefficient can be obtained from the multiple-scattering expression described in Eq. (6):

$$
t_{0}=\tau^{12} e^{i k_{\omega} h} \tau^{23} /\left(1-e^{2 i k_{\omega} h} \rho^{2}\right),
$$

where $t_{0} \equiv t_{0 p}, \tau^{12} \equiv \tau_{0}^{12}, \rho \equiv \rho_{00}^{23}=\rho_{00}^{21}$, and $\tau^{23} \equiv \tau_{0,0}^{23}$. All these scattering magnitudes can be related to $G \equiv G_{00}$ $=i \Sigma_{\vec{k}} k_{\omega} / k_{z}|\langle 0 \mid \vec{k}\rangle|^{2}$, where the sum runs over all wave vectors of the form $\vec{k}=\vec{k}_{0}+\vec{k}_{R}$, with $\vec{k}_{0}$ the parallel component of the incident wave vector and $\vec{k}_{R}$ a wave vector of the $1 \mathrm{D}$ reciprocal lattice:

$$
\rho=-\frac{G-i}{G+i}, \quad \tau^{12}=\frac{I_{0}}{G+i}, \quad \tau^{23}=\frac{2 i\left\langle\vec{k}_{0} p \mid 0\right\rangle}{G+i},
$$

where the overlap integral is $\langle\vec{k} p \mid 0\rangle=\sqrt{a / d} \operatorname{sinc}(k a / 2)$ and the illumination term $I_{0}=2 i Y_{\vec{k}_{0} p}\left\langle\vec{k}_{0} p \mid 0\right\rangle$. The complex two-media reflection coefficient $\rho$ is characterized by its modulus $R$ and phase $\theta$. Then, we can define a total phase $\phi=2 \theta+2 k_{\omega} h$ as the sum of the scattering (associated with $\rho$ ) and geometrical (determined by the optical path) phases. As a function of this total phase, the zero-order transmission coefficient in Eq. (15) can be expressed as

$$
t_{0}=\tau^{12} e^{i k_{\omega} h} \tau^{23} /\left(1-R^{2} e^{i \phi}\right) .
$$

Equation (17) resembles the formula governing the transmission properties of a Fabry-Perot interferometer. In contrast to the canonical case in which the phase of the EM fields inside the cavity is determined by the optical path $\left(2 k_{\omega} h\right)$, it also contains the scattering phase shift that sometimes presents a strong spectral dependence. Figure 16 analyzes the behavior of $R$ and $\theta$ for three different values of $a / d$. Both the modulus and the phase of $\rho$ present singular behavior at wavelengths $d$, $d / 2, d / 3, \ldots$. This is because $G$ diverges at the condition $k_{z}=0$ which, for normal incidence, coincides with $\lambda$ $=d / n$, with $n$ an integer. At those particular wavelengths, Eq. (16) predicts $\tau^{12}=\tau^{23}=0$ and $\rho=-1$, provoking the appearance of zeros in the transmittance, as 


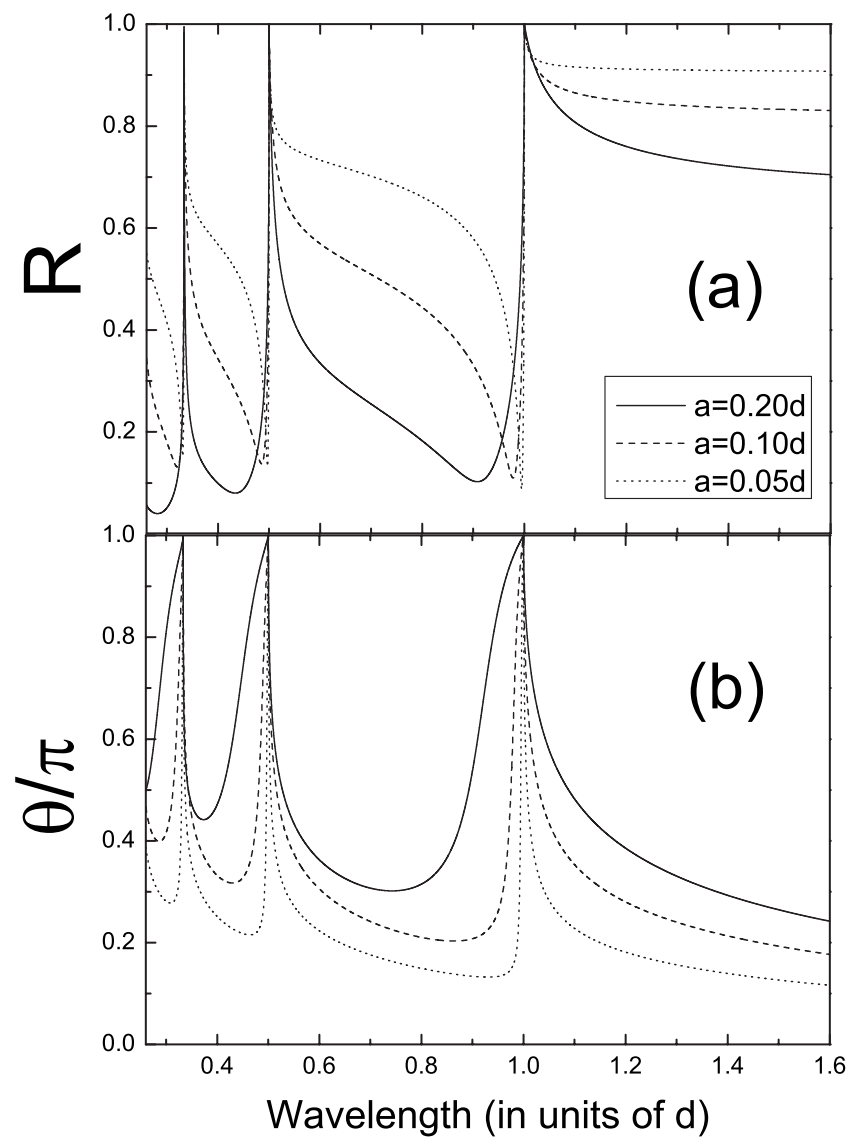

FIG. 16. Dependence of $\rho$ vs wavelength for three different values of the ratio between the width of the slits and period of the array, $a / d$. (a) The modulus of $\rho(R)$ and (b) its phase $(\theta)$, in units of $\pi$.

shown in Fig. 15. An interesting point is that there is a phase jump between 0 and $\pi$ in $\rho$ when $\lambda$ crosses those singular conditions (see Fig. 16).

Given that for narrow slits $R \approx 1$ there is a transmission peak, associated with a resonant condition for the denominator in Eq. (17), whenever $\phi$ is an integer times $2 \pi$. For thin PEC films, the total phase is dominated by the scattering phase; correspondingly, the spectral location of the transmission resonance appears at a wavelength slightly longer than $d$ (see the case $h=0.2 d$ in Fig. $15)$. When $h$ is increased, the situation is more complex and the geometrical phase $\left(2 k_{\omega} h\right)$ can also lead to the appearance of transmission peaks emerging at $\lambda \gg d$. The physical origin of these transmission resonances stems from the excitation of cavity resonances similar to the ones emerging in single slits as discussed in Sec. II.A.

However, there is a fundamental question regarding the origin of the transmission peak at $\lambda$ close to $d$. Notice that, up to this point, we have discussed the situation within the PEC approximation and, hence, there are no SPPs involved. However, in a PEC film, when the metal is perforated with a periodic array of indentations, surface EM modes can build up (Munk, 2000). We now describe the link between the transmission resonances previously reported and the existence of surface EM modes in the structure.
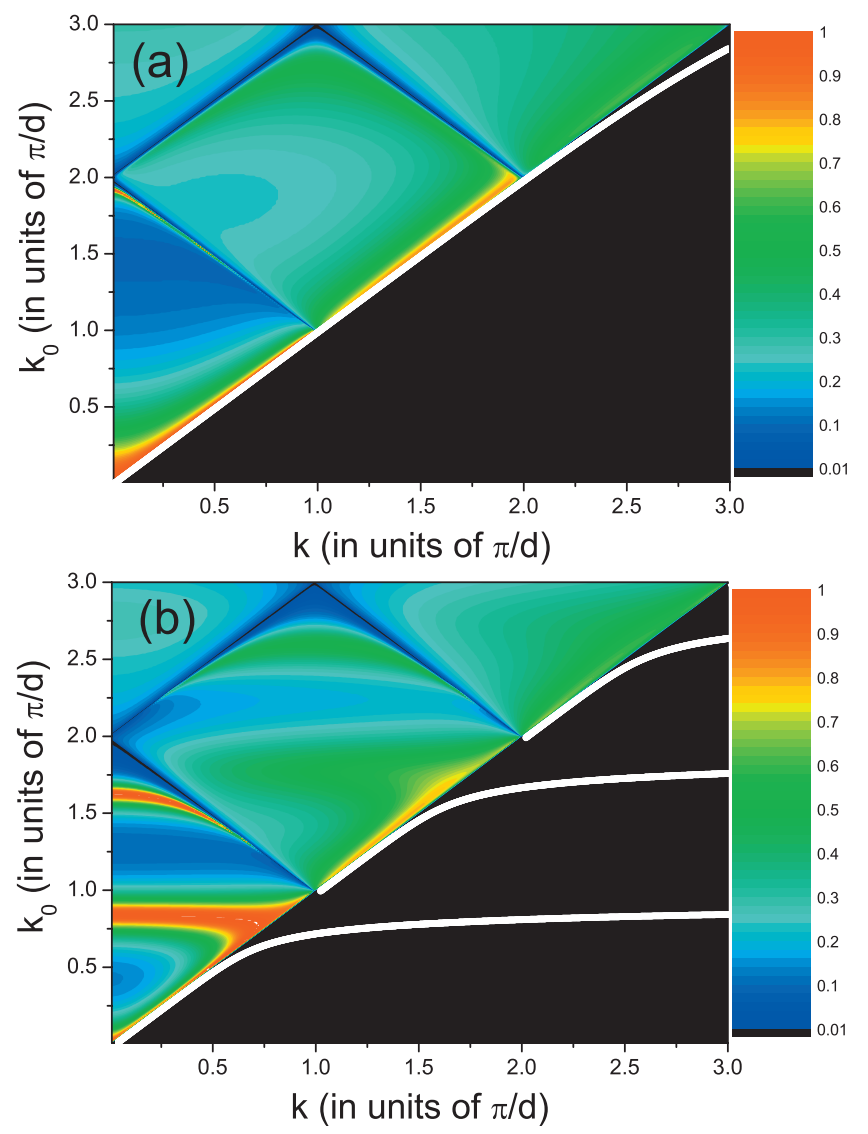

FIG. 17. (Color) Transmission spectra versus dispersion relations of the surface EM modes. Left panels: Transmittance spectra as a function of both wave number and wave vector for $p$-polarized light impinging at two different arrays of slits. Right panels: Dispersion relation for the EM guided modes as obtained from Eq. (18). (a) $a=h=0.2 d$. (b) $a=0.2 d$ and $h$ $=1.0 \mathrm{~d}$.

The surface EM modes relevant to the transmission process cannot be truly bound modes; they must be leaky modes, as otherwise they could not be excited by an incident plane wave. However, in a first approximation, we can look for the existence of truly bound surface EM modes by working with a parallel momentum $k_{x}$ larger than $k_{\omega}$ and ignoring diffraction effects (which are incorporated in a second step). The dispersion relation (frequency versus $k_{x}$ ) of the $p$-polarized EM modes supported by the structure can be calculated using Eq. (3) as is done in the Appendix, leading to the following equation:

$$
\frac{\sqrt{k_{x}^{2}-k_{\omega}^{2}}}{k_{\omega}}=\frac{a}{d} \frac{\sin k_{\omega} h}{\cos k_{\omega} h \pm 1},
$$

where the sign (+) must be taken when $\sin k_{\omega} h>0$ and $\operatorname{sign}(-)$ when $\sin k_{\omega} h<0$. This equation gives the dispersion relation of the bound EM modes supported by the structure within the effective medium limit $(\lambda \gg d, a)$.

Figure 17 shows the close link between the spectral locations of these EM modes and the peaks in the trans- 
mittance spectrum as a function of $k_{x}$. In Fig. 17(a) we analyze the case $h=0.2 d$. Here the dispersion relation (white line) runs close to the light line $\left(\omega=c k_{x}\right)$. The important point to realize is that if $\lambda<2 d$ and diffraction effects are taken into account, these EM modes become leaky and can be excited by an impinging propagating plane wave. The left part of Fig. 17(a) shows the transmittance versus wave number and $k_{x}$ within the light cone. It is clear that the location of the transmission peak at $\lambda \approx d$ at normal incidence can be obtained by folding the dispersion relation inside the light cone. Therefore, we conclude that the physical origin of the transmission peak located at $\lambda \approx d$ is related to the excitation of an EM bound mode. As fields at the two horizontal metal surfaces of the film are always connected via a propagating wave (the TEM mode), this mode has an $E$ field that may oscillate inside the slits. In this way, it resembles more the guided mode in a dielectric slab than a SPP. In fact, it has been demonstrated that a 1D array of subwavelength slits can be considered as a metamaterial and can be rigorously mapped into a highrefractive-index dielectric slab (Shen et al., 2005). We will show later that the case of a $2 \mathrm{D}$ hole array is different: the geometrically induced EM modes are truly surface EM modes due to the existence of a cutoff wavelength in 2D apertures (see Sec. III.B.2). The close correspondence between the dispersion relation of the EM modes and transmission peaks also occurs for thicker films [see Fig. 17(b), $h=1.0 d$ ]. For large enough $h$, the dispersion relation presents flat parts, associated with the Fabry-Perot condition $\cos k_{\omega} h= \pm 1$, which dominate the transmission spectrum.

What occurs to these transmission resonances when the metal is not a PEC? As explained before, within the coupled-mode formalism, the dielectric function of the metal can be approximately considered by using the surface impedance boundary conditions when the EM fields are matched at the two horizontal interfaces of the structure. In this way, the system of equations (1) still holds but now the different magnitudes depend on the surface impedance of the metal $Z_{S}$ (see the Appendix). The most important change resides in the propagator $G_{\alpha \beta}$, which in the single-mode approximation $G=G_{00}$, reads

$$
G=i \sum_{\vec{k}} \frac{k_{\omega}}{k_{z}+Z_{S} k_{\omega}}|\langle 0 \mid \vec{k} p\rangle|^{2} .
$$

The divergence of $G$ now appears at the condition $k_{z}+Z_{S} k_{\omega}=0$, which is the condition for the existence of a SPP on a flat metal surface within the SIBC approach. As the divergence of $G$ marks the spectral location of the deep transmission minimum, now this minimum will not emerge at $\lambda=d$, as in the case of a PEC film, but at a slightly longer wavelength. Therefore, this transmission peak is associated with the excitation of a guided EM mode whose dispersion relation has both dielectric (linked to a finite $\epsilon$ ) and geometric contributions (due to the presence of the slits). The linewidths of these transmission resonances are much smaller than the FabryPerot ones and therefore they are much more sensitive to the presence of absorption in a metal at optical frequencies (Garcia-Vidal and Martin-Moreno, 2002) and finite-size effects (Fernandez-Dominguez et al., 2007).

\section{Experimental results}

As stated above, there have been far fewer experimental studies on the transmission properties of 1D arrays of slits than of 2D arrays of holes. In the microwave regime, Sambles and co-workers (Went et al., 2000; Hibbins et al., 2001) reported the appearance of selective transmission at resonant wavelengths associated with the excitation of cavity resonances. Barbara et al. (2002) presented the first study in the optical regime with goldcoated silica gratings and they were able to interpret their results by invoking the excitation of both SPPs and slit-cavity resonances. Two different experimental groups (Steele et al., 2003; Sun et al., 2003) reported the emergence of transmission resonances associated with the excitation of SPPs in very thin metallodielectric gratings. The transmission properties of terahertz radiation pulses were studied in very thick transmission gratings containing subwavelength slits by Xing et al. (2006).

Recently Pang et al. (2007) measured the dispersion relation of the transmission resonances appearing in $1 \mathrm{D}$ arrays of subwavelength slits in both the visible and near infrared (NIR) ranges of the EM spectrum. Their experimental transmission spectra, shown in Fig. 18, corroborate the theoretical predictions regarding the existence of two different channels that enhance the transmission through a 1D periodic array of slits. As the SPP dispersion relation is close to the light line in this frequency range, the resonant features (maximum and deep transmission minimum) associated with the SPPs of the metal-substrate interface appear close to the Rayleigh condition $\lambda \approx d \sqrt{\epsilon_{\text {glass }}}=1125 \mathrm{~nm}$. On the other hand, the spectral location of the slit cavity resonance is expected to appear at a wavelength larger than $2 h=800-900 \mathrm{~nm}$. The two main transmission peaks emerging in the spectrum result from the strong interplay between these two types of resonances, as described previously. This interaction is better visualized in Fig. 18(b) where a dispersion diagram of the transmission peaks as a function of parallel momentum is presented. The SPP mode of the glass-metal interface $(\mathrm{SM}-1)$ overlaps with the cavity resonance at ca. $1.1 \mathrm{eV}$ (marked by a dashed line, FP), provoking the opening of a gap of approximately $280 \mathrm{meV}$. Full lines display the dispersion relation of the different SPP modes of the flat interfaces.

Similar transmission resonances have also been reported in transmission gratings perforated on doped semiconductors at terahertz frequencies (Gomez Rivas et al., 2005; Parthasarathy et al., 2007) and on SiC films at NIR wavelengths (Marquier et al., 2004). In this last case, surface phonon polaritons play the same key role as SPPs do in the metallic case. Finally, some interesting transmission properties emerging in compound gratings (formed by slit arrays in which the unit cell is composed by more than one slit) have recently been reported (Skigin and Depine, 2005, 2006; Fong and Hui, 2006; Hib- 

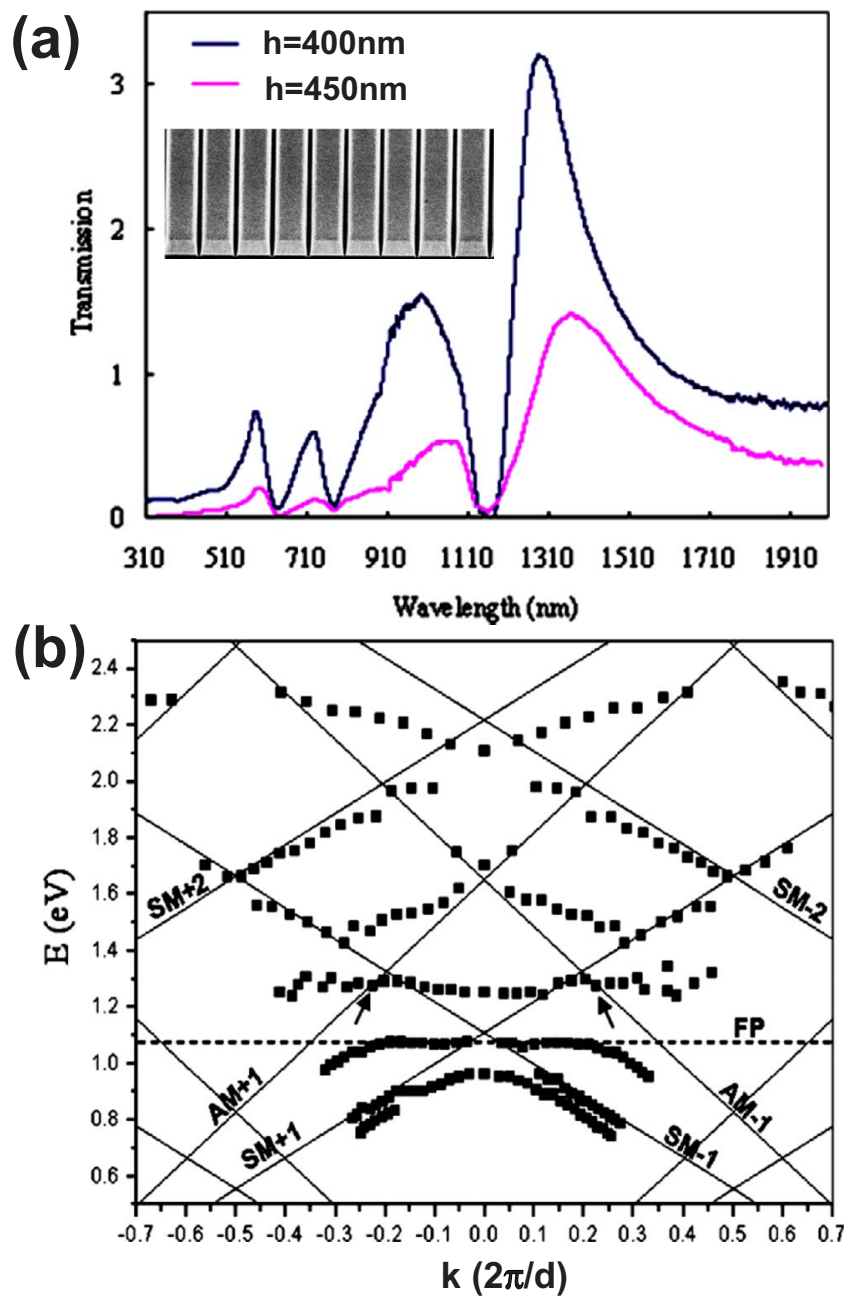

FIG. 18. (Color online) Transmission through 1D periodic arrays of slits. (a) Inset: SEM image of one of the $1 \mathrm{D}$ arrays analyzed in these experiments. The period of the array is $d$ $=750 \mathrm{~nm}$, the width $a=100 \mathrm{~nm}$, and two different thicknesses are analyzed, $h=400$ and $450 \mathrm{~nm}$. Main panel: Zero-order transmittance of a 1D slit array illuminated by a normal incidence $p$-polarized plane wave. (b) Dispersion diagram [spectral locations of the transmission peaks vs energy (in eV) and parallel momentum (in units of $2 \pi / d$ )] for the sample analyzed in panel (a) with $h=400 \mathrm{~nm}$. From Pang et al., 2007.

bins et al., 2006). By increasing the number of different slits within the unit cell, new phase resonances appear as sharp dips within the transmission maxima associated with the excitation of Fabry-Perot modes.

\section{B. Two-dimensional periodic arrays of holes}

\section{General results}

The first observation of the EOT phenomenon in subwavelength hole arrays was made on 200-nm-thick gold and silver films perforated with a square lattice of circular subwavelength holes (Ebbesen et al., 1998). Figure 19 presents a SEM image of a 2D hole array exhibiting EOT. A typical EOT transmission spectrum is characterized by a sequence of maxima and minima (see Fig. 20). In the first set of experiments, transmissions twice as

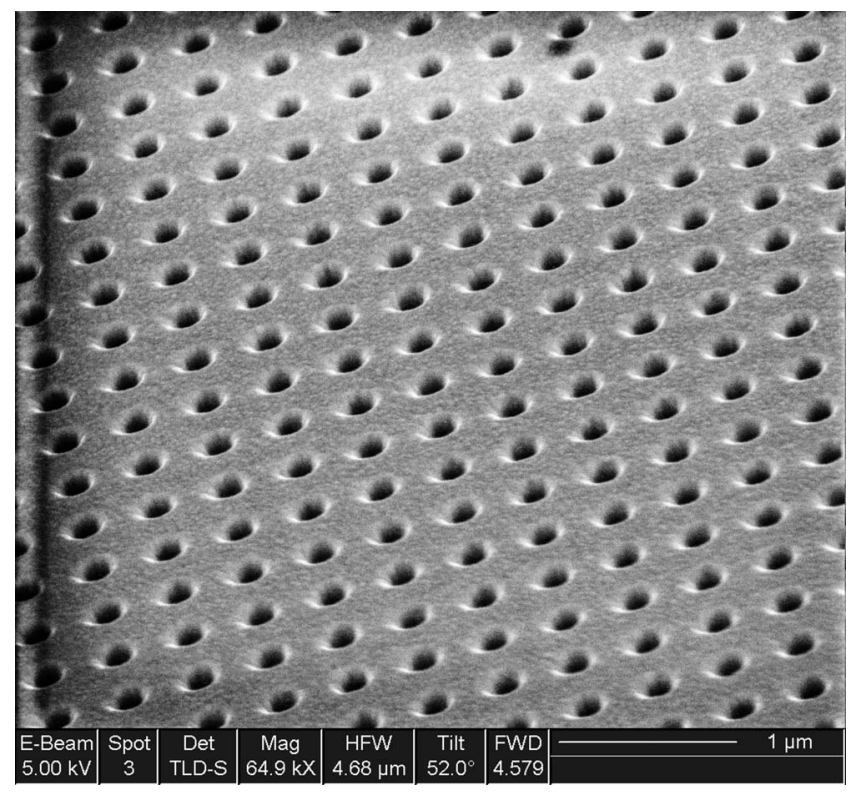

FIG. 19. SEM image of a 2D hole array of circular holes (diameter of $150 \mathrm{~nm}$ ) milled in a 260-nm-thick Au film that is deposited on a glass substrate. The hole arrays count $30 \times 30$ holes and the period of the square array is $460 \mathrm{~nm}$. Courtesy of Eric Laux.

high as in the open-air fraction were observed. This observation was all the more surprising as conventional theory for light transmission through a single subwavelength hole predicts a transmission normalized to the hole area that is substantially less than 1 (see Sec. II.B.1).

The discovery of the EOT phenomenon sparked a large amount of research trying to unveil the underlying

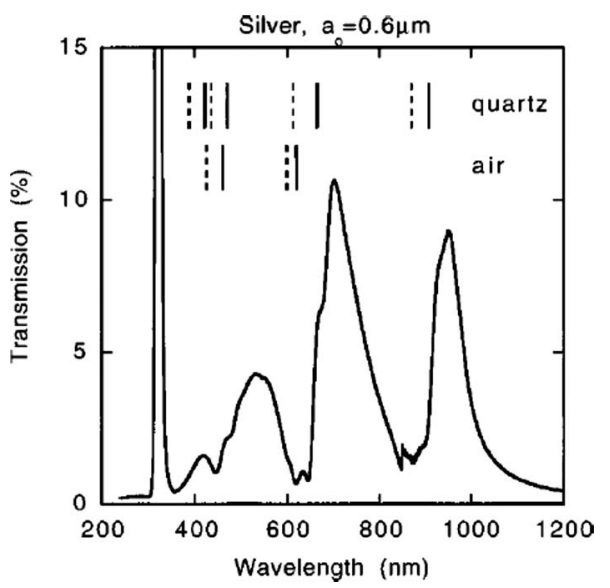

FIG. 20. Zero-order transmission of subwavelength hole array in a $\mathrm{Ag}$ film on a quartz substrate for normal incidence; the lattice period is $600 \mathrm{~nm}$, the film thickness is $200 \mathrm{~nm}$, and the diameter of the circular holes is $150 \mathrm{~nm}$. The markers indicate positions for the maxima and minima expected for SPP modes on either the silver-quartz or the silver-air interface (solid markers) and the Wood-Rayleigh anomalies associated with the same interfaces (dashed markers). From Ghaemi et al., 1998. 
mechanisms. The first report (Ebbesen et al., 1998) presented dependencies on hole size, lattice spacing, metal film thickness, and angular dispersion. Later, several other important parameters and observations were made: the type of metal, the role of symmetry in the dielectric-metal-dielectric layer stack, the finite-size effects of the lattice, and the role of the hole shape.

The first key finding was the spectral dependence on the lattice period of the minima and maxima for transmission. The positions of the minima exhibit a linear dependence on the lattice period. In the first experimental report, the minima were ascribed to the spectral locations where a diffraction order is exactly parallel to the plane of the metal film, the so-called Wood-Rayleigh anomaly. On the other hand, transmission maxima exhibit almost the same scaling behavior with lattice period as the minima but appear slightly redshifted. Ghaemi et al. (1998) suggested that maximum transmission occurs for those optical frequencies for which the parallel component of the wave vector of a SPP is equal to a wave vector of the $2 \mathrm{D}$ reciprocal lattice associated with the geometry of the array. That is, the transmission maxima approximately satisfied the following condition:

$$
\vec{k}_{\mathrm{SPP}}=\vec{k}_{\mathrm{in}}+\vec{k}_{R}
$$

where $\vec{k}_{\mathrm{SPP}}$ and $\vec{k}_{\text {in }}$ are the parallel components of the wave vectors of the SPP and the incident plane wave, respectively; $\vec{k}_{R}=n \vec{b}_{1}+m \vec{b}_{2}$ is a wave vector of the $2 \mathrm{D}$ reciprocal lattice (expressed as a linear combination of the primitive vectors $\vec{b}_{1}$ and $\vec{b}_{2}$ ).

The dispersion relation for a SPP on a flat metaldielectric interface is given by

$$
k_{\mathrm{SPP}}=\frac{\omega}{c} \sqrt{\frac{\epsilon_{M} \epsilon_{d}}{\epsilon_{M}+\epsilon_{d}}}
$$

where $\epsilon_{M}$ and $\epsilon_{d}$ are the electric permittivities of the metal and dielectric, respectively.

Notice that for $\left|\epsilon_{m}\right| \gg \epsilon_{d}, k_{\mathrm{SPP}} \approx k_{\mathrm{ph}}$, where $k_{\mathrm{ph}}$ is the photon wave vector in the dielectric. This implies that, in a good metal, the spectral location of the WoodRayleigh anomaly is close to the condition for excitation of a SPP on a flat metal-dielectric interface. As there are SPP modes bound to both metal-dielectric interfaces, two sets of transmission maxima occur when the dielectrics on either side of the metal film are different. As seen in Fig. 20, the assignment of the maxima to SPP excitation and of the minima to the Wood-Rayleigh anomalies done in the first studies of EOT in 2D hole arrays was sound but not completely accurate.

The resonance condition given by Eq. (20) directly relates the angle of incidence and the spectral locations of both the maxima and the minima. Figure 21 shows the measured transmission through a hole array as a function of the angle of incidence (Ghaemi et al., 1998). Simple goniometry suffices to convert wavelength and tilt angle of the sample with respect to the optical axis into an in-plane wave vector. It is clear that both minima

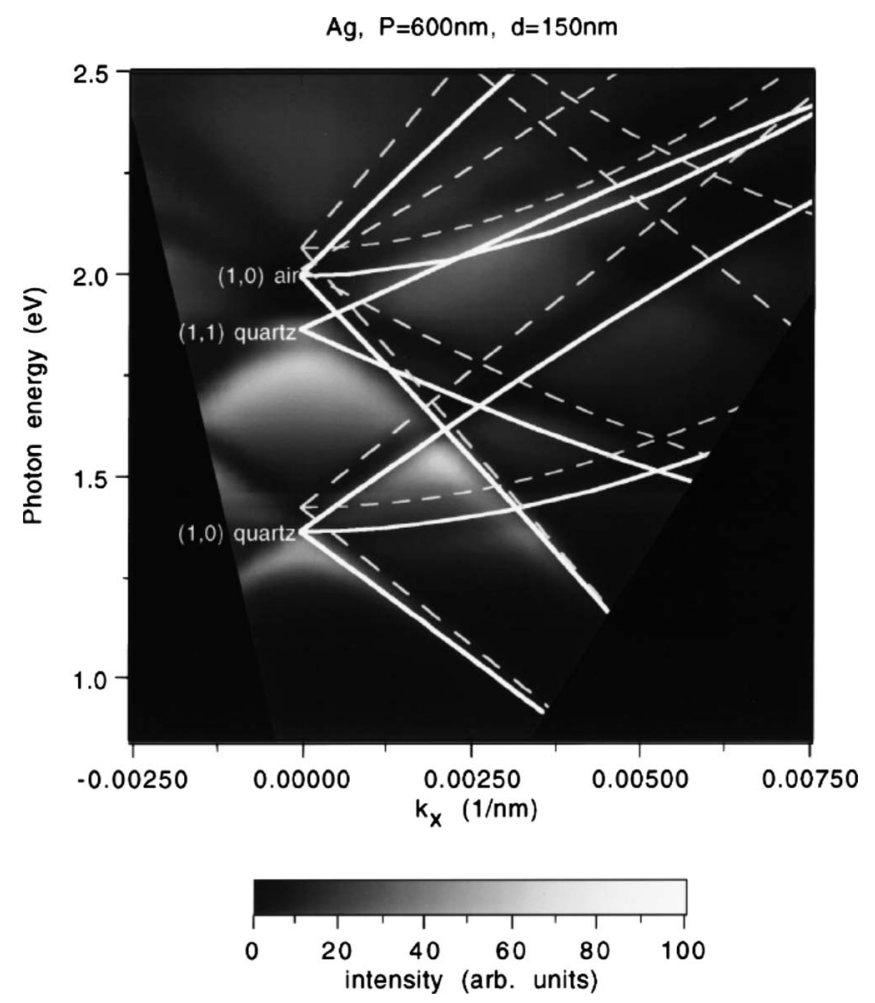

FIG. 21. Measured and predicted dispersion of the transmittance through a subwavelength hole array. The transmitted intensity is given by a gray scale. The loci of the maxima (full lines) related to the excitation of SPPs on a flat surface and the minima ascribed to the Wood-Rayleigh anomaly (dashed lines) are superimposed on the measured data. From Ghaemi et al., 1998.

and maxima exhibit dispersion, presenting a reasonable agreement with the values expected from the aforementioned assignment scheme.

This was the physical picture provided by the group that discovered EOT phenomenon in their first two papers published in 1998. Here we summarize the subsequent developments in the search for a better understanding of the EOT phenomenon. After that, we present a more detailed view based on the coupledmode method used throughout this review.

The first theoretical calculations were performed with the RCWM (Popov et al., 2000). This group concluded that the EM fields within a hole drilled in a metal film decay much more slowly when the holes form a 2D array than would be expected from estimations based on the hole size. In keeping with the first experimental results, the calculated EOT features are closely matched to the remapping of SPP bands within the light cone. However, the existence of EOT in holey PEC films (Martin-Moreno et al., 2001) showed that the presence of waveguide modes with "modified" propagation constants is not essential for observing EOT.

In this last reference, EOT features were explained in terms of the admittances (inverse of impedances) of the waveguide modes and the effective admittances of the two semi-infinite media, as seen from the waveguide modes. This effective admittance is an average of the 
overlap between the waveguide mode and all diffraction modes, weighted by the admittance of the latter, which plays the role of a density of states. The main point is that this "density of states" diverges whenever one of the diffraction orders coincides with a SPP with the corresponding wave vector or, in a PEC, when one of the diffraction orders becomes tangent to the surface. The existence of different conditions for real metals and PEC already points to the existence of two sources for the effective impedance of a corrugated surface: dielectric (due to the dielectric constant of the metal) and geometric (due to the corrugations). In a PEC only the geometrical source plays a role, while in a real metal both terms are present. As mentioned, transmittance minima are related to divergencies of the effective admittance, which arise due to constructive reillumination of a hole by all other holes in the array (Bravo-Abad, GarciaVidal, and Martin-Moreno, 2004; De Abajo et al., 2005; Genet et al., 2005; De Abajo, 2007). Actually, the effective admittance only diverges in a lossless metal but reaches very high values for the case of good metals such as gold and silver even in the optical regime. With this caveat in mind and for reasons of economy of language, we keep loosely referring to these high values as divergences. Exactly at the divergence the transmittance vanishes as the incident field is short circuited into the responsible diffraction order and, therefore, does not see the holes. However, at frequencies close to the divergence, the effective admittance changes rapidly and, eventually, equals the admittance of the waveguide modes. This signals the presence of a surface EM mode. Surface EM modes at both sides of the film are coupled through the evanescent fields inside the hole, and the resulting coupled resonant modes give rise to transmission maxima. These surface EM modes were termed "SPPs of the corrugated surface," as they appear in metals and involve both EM fields and surface currents. However, this terminology may have led to some confusion because these modes appear also for a PEC, where the uncorrugated surface does not support SPPs. The interpretation of the EOT process in terms of impedance (or admittance) matching has been recently reformulated by Medina et al. (2008) by means of a transmission-line circuit model similar to those used within the microwave engineering community and whose parameters (capacitances and inductances) are fitted to numerical simulations.

The physical picture in which the EOT phenomenon in hole arrays is due to the excitation of surface EM modes has been corroborated by more sophisticated (virtually exact) calculations, able to deal with the dielectric response of metals at optical frequencies (Lalanne, Rodier, and Hugonin, 2005). They were able to match the spectral locations of transmission maxima and minima with those corresponding with the SPPs supported by the holey metal film (see Fig. 22).

The resonant nature of the EOT phenomenon has also been confirmed by different experimental studies. Krishnan et al. (2001) showed that this resonant process is optimal when, for a given angle of incidence, the sur-

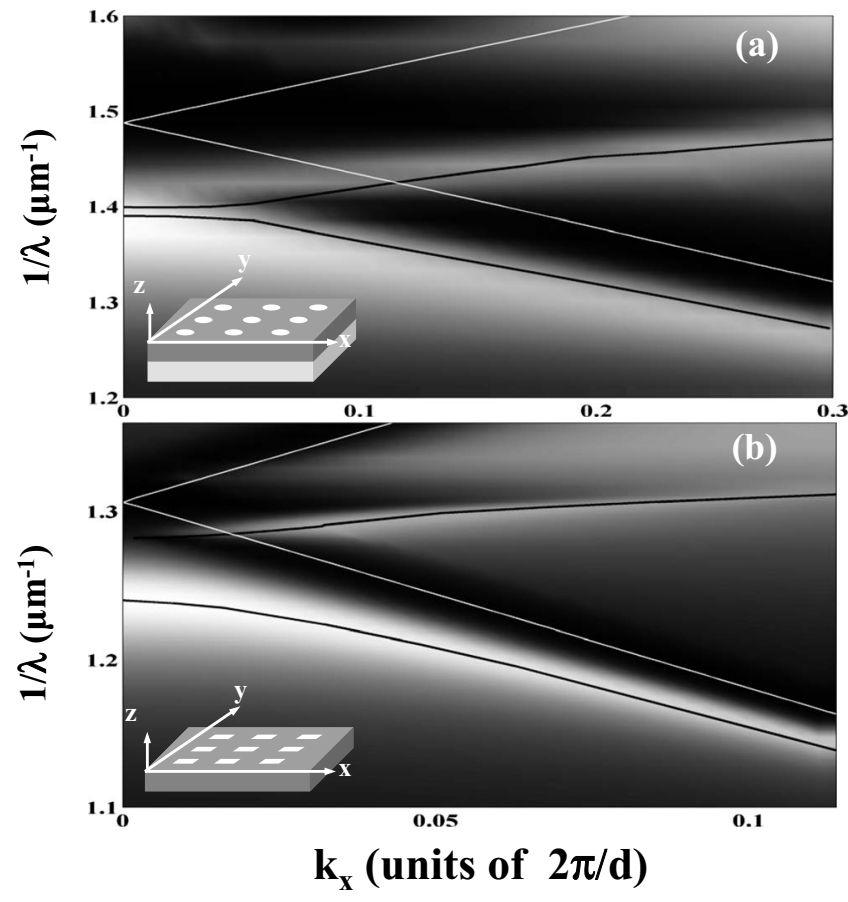

FIG. 22. Gray-scale images show the zero-order transmittance for two holey silver films as a function of the frequency and the in-plane wave vector $k_{x}$. (a) Circular hole geometry for a silver film on glass. The scale is linear and the maximum transmittance is $32 \%$. (b) Square hole geometry for a silver freestanding film. The maximum transmittance is $50 \%$ in this case. Superimposed black curves correspond to the SPP dispersion relations of (a) glass-silver interfaces perforated by circular holes and (b) air-silver interfaces drilled with square holes. The white curves correspond to the SPP dispersion relation for a flat interface: (a) glass-silver and (b) air-silver. From Lalanne, Rodier, and Hugonin, 2005.

face EM modes of both surfaces are maximally coupled. This occurs in the symmetric environment of equal dielectric constants in the regions of incidence and transmission, as shown in Fig. 23. An additional property associated with the excitation of these coupled surface EM modes is that the corresponding EM fields are greatly enhanced at the surface (Salomon et al., 2001). The lower three panels of Fig. 23 present calculations showing the large increase of $E$-field intensities at the metaldielectric interfaces that accompany EOT resonances. This point will be further elaborated in Sec. III.B.5. By comparing the results of the transmitted, reflected, and absorbed power associated with the EOT process in $2 \mathrm{D}$ hole arrays, Barnes et al. (2004) were able to conclude that SPP modes act to enhance the EM fields at the metal surface, thus providing a way for enhancing the transmittance through the subwavelength holes.

The view that EOT in hole arrays is due to coupled surface EM modes has been criticized by Lezec and Thio (2004). They performed experiments in arrays of $9 \times 9$ holes in both metal and absorbing dielectric films (such as W or amorphous $\mathrm{Si}$ ) and analyzed the per-hole transmission enhancement with respect to that of isolated holes. The maximum enhancement measured was 

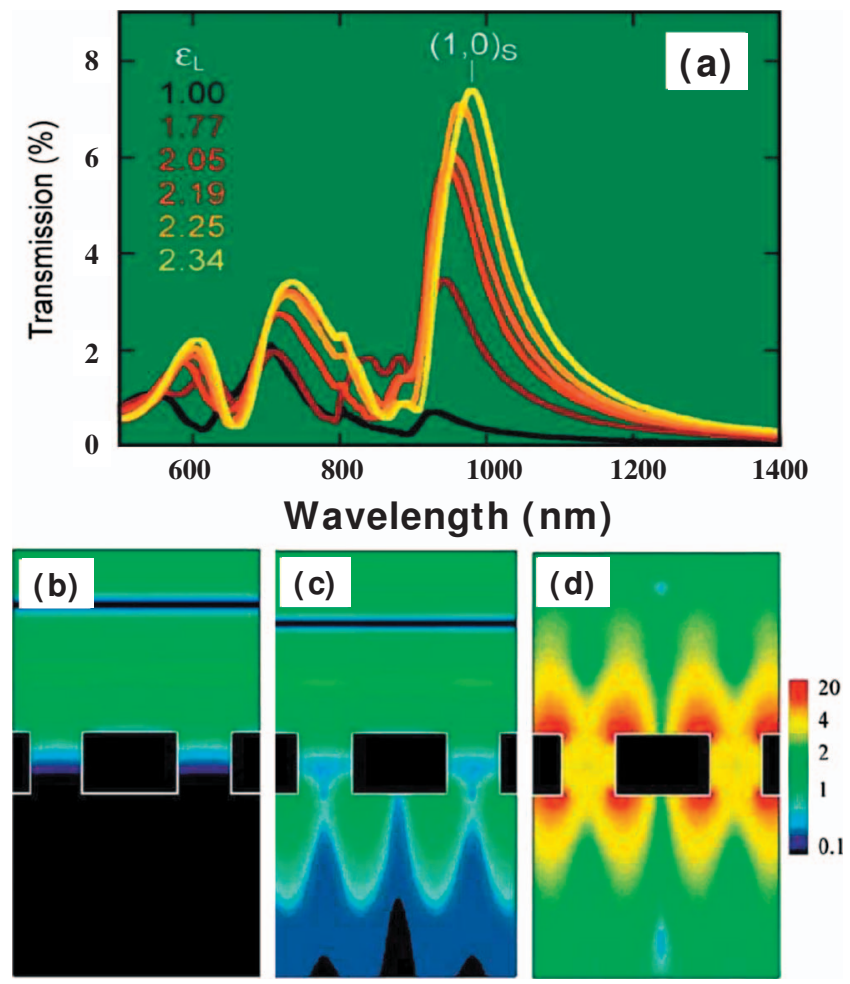

FIG. 23. (Color) Transmission through 2D periodic arrays of holes. (a) Experimental zero-order transmittance of a gold film on a quartz substrate $\left(\epsilon_{s}=2.31\right)$ as a function of the dielectric constant of the superstrate $\epsilon_{L}$. The film thickness is $250 \mathrm{~nm}$, the hole diameter is $200 \mathrm{~nm}$, and the period of the square array is $600 \mathrm{~nm}$. (b)-(d) Calculated cross-sectional views of the electric field amplitudes with various states of resonance: (b) off resonance in the asymmetric situation (transmission $0.005 \%$ ), (c) on resonance in the asymmetric situation (transmission $0.5 \%$ ), and (d) on resonance in the symmetric situation (transmission $20 \%$ ). In the three cases, light is impinging from the top and the $E$-field amplitude is normalized to the amplitude of the incident plane wave using a logarithmic color scale. From Krishnan et al., 2001.

about 7 for the metal case and ten times smaller for the dielectric case. As dielectrics do not support SPPs, they concluded that surface EM modes could not be responsible for the transmission enhancement. Subsequent works have found larger differences between enhancements in holey metals and dielectrics [see, for instance, Przybilla, Degiron, et al. (2006)] and that the per hole transmission enhancement in holey metals is virtually unlimited for larger arrays [see Przybilla et al. (2008) and Sec. III.B.6]. Additionally, theoretical studies (Sarrazin and Vigneron, 2003) showed that the small optical transmission enhancements in $\mathrm{W}$ are still due to the weakly bound surface EM modes, known as Zenneck waves, which appear at the interface between air and a lossy dielectric (Zenneck, 1907). The different confinements of SPPs and Zenneck waves explain why transmission resonances in metals and dielectrics present large differences in both maximum enhancement and peak visibility (defined as the difference between the peak maximum and peak minimum divided by their sum).
A possible origin of the criticism of the relevance of coupled surface EM modes to EOT may be the incorrect association of the relevant surface EM modes with those of an uncorrugated surface. Also, the appearance of EOT when the metal is considered as a PEC has also led to some doubts about the relevance of coupled surface EM modes. However, periodically corrugated PEC surfaces do support surface EM modes (Munk, 2000) which, in a perforated film, assist the transmission process (Pendry et al., 2004). The link between EOT in real metals and in perfect conductors was more evident after it was found that the structured PEC surface could be approximately represented by effective permeabilities that copy those of a Drude-like metal (Pendry et al., 2004; GarciaVidal, Martin-Moreno, and Pendry, 2005). The emergence of these surface EM modes in holey slabs (which are now commonly called spoof SPPs) is a complex phenomenon, which involves the multiple scattering of the EM fields between both surfaces and holes at the same surface.

Even the direct EM coupling between two holes in a metal surface is already a complex phenomenon that arises from the interference of both radiative and evanescent diffraction modes, governed by what are known as Sommerfeld integrals [see, for instance, Felsen and Marcuvitz (2003)]. In a real metal, the interhole coupling presents different regimes as a function of the distance between holes and the type of metal considered. We describe the coupling behavior for good metals such as $\mathrm{Ag}$ or $\mathrm{Au}$ in the optical regime. At small distances (significantly smaller than the wavelength) all diffraction modes contribute and the coupling is similar to what it would be in a PEC. At distances longer than roughly two to three wavelengths, most diffracted EM fields cancel each other at the surface and the coupling through SPPs takes over up to distances around 20-30 $\mu \mathrm{m}$ (Sondergaard and Bozhevolnyi, 2004; López-Tejeira et al., 2005). EM coupling in the intermediate regime comes from complicated interferences; exact computations have shown that it can be phenomenologically described as a SPP plus a rapidly attenuated contribution (termed a creeping wave) that oscillates with the free-space wavelength (Lalanne, Hugonin, and Rodier, 2005). Recently the details of the formation of leaky surface EM waves and transmission resonances from the direct interhole coupling have been addressed theoretically (Liu and Lalanne, 2008). Additionally, this calculation sheds light on the relevance to the EOT phenomena of the different channels (radiative fields, evanescent fields, SPPs, etc.) that transfer EM fields for both PEC and real metals at optical frequencies. An important result that emerges from theory is that the formation of surface EM modes in holey metal surfaces is due to the multiple scattering of EM fields between holes (Martin-Moreno et al., 2003; De Abajo and Saenz, 2005; Liu and Lalanne, 2008). The precise form of the direct interhole coupling affects the energy of the modes, but it is of no great relevance to their existence. This is why these surface EM modes appear both in a real metal (where the direct coupling between holes is mostly through the SPPs of the flat sur- 
face) and in a PEC (where all coupling is via diffraction modes). This important point is absent in the composite diffraction evanescent wave (CDEW) model introduced by Lezec and Thio (2004) when interpreting their experimental results. The CDEW model considers only the direct interhole coupling coming from evanescent diffracted waves in a scalar model for a PEC. By addition of some fitting parameters (notably the coupling strength and the in-plane wave vector) the model provided reasonable fits with experimental data on the optical transmission through sets of apertures. Lezec and Thio (2004) concluded that the origin of EOT did not reside in SPPs as their model did not take SPPs into account. It was shown later that the CDEW model ignores important aspects of polarization and neglects contributions to the diffracted field (Garcia-Vidal, Rodrigo, and Martin-Moreno, 2006; Lalanne and Hogonin, 2006; Visser, 2006). Additionally, CDEW analysis takes into account only first-order processes where each aperture scatters the EM fields just once, neglecting the multiple scattering of fields by the apertures, which are essential for understanding EOT.

To summarize, nowadays there is a wide consensus that the physics of EOT in 2D hole arrays is that of two surface EM modes weakly coupled between themselves and weakly coupled to a continuum. Actually, the hybridization of the two weakly coupled surface EM modes originates leaky bound EM modes that act as almost independent channels. Therefore, in general, EOT is expected to appear even when only a leaky surface EM mode is weakly coupled to a continuum. This idea is useful to direct the search of EOT to other ranges of the EM spectrum, to other types of material presenting surface EM modes (such as polar semiconductors with surface phonon polaritons), and even to other types of waves. Note that, close to the cutoff of TM modes, leaky surface modes also appear in perforated diaphragms on metallic waveguides. Correspondingly, EOT also emerges in these confined systems (Gordon, 2007; Medina et al., 2008). The link between EOT and surface modes is also useful because it allows for a phenomenological description of the (sometimes very asymmetric) line shapes of transmission resonances in terms of the Fano model (Fano, 1961). Here we reproduce the argument [borrowed from the theory of reflection gratings (Neviere, 1980)] given by Enoch et al. (2002) and Sarrazin et al. (2003). The scattering matrix is dominated by divergencies (simple poles) that occur when the incident field excites a resonance in the system, in this case a surface EM mode. These resonances are leaky, as they couple to radiative modes. This means that the poles appear at complex frequencies or, alternatively, at complex wavelengths. We focus first on the case in which one of these poles (characterized by a complex wavelength $\lambda_{\text {pole }}$ ) dominates a given region of the spectrum. Now imagine that we consider systems with smaller holes. Obviously, when the holes are not present, the diffraction coupling to the surface EM mode vanishes and the resonance in the scattering matrix must disappear. Analytical continuity implies that there must be a zero in the scattering matrix (characterized by a complex wavelength $\lambda_{\text {zero }}$ ) which, in the limit of vanishing hole size, coincides with the spectral position of the pole. Therefore, close to resonance, the transmittance must be of the form

$$
T(\lambda)=T_{\mathrm{bg}}\left|\lambda-\lambda_{\text {zero }}\right|^{2}\left|\lambda-\lambda_{\text {pole }}\right|^{2},
$$

where $T_{\mathrm{bg}}$ represents the background (nonresonant) contribution. As a technical note, this argument applies to the case of a real conductor, where the transmission through the unperforated film can be arbitrarily small but is not zero. The zero-thickness PEC case is pathological in the sense that $T_{\mathrm{bg}}$ must vanish when the size of the hole goes to zero and this does not occur if the film thickness also approaches zero (see the discussion in the next section). The argument outlined above still applies if the zero-thickness PEC limit (infinitely small hole sizes, infinitely thin film, and infinitely large dielectric constant of the metal) is analyzed by taking first both the hole size and film thickness to zero and then the dielectric constant to infinity. Numerical computations for the case of a PEC confirm that Eq. (22) still applies, with $\lambda_{\text {zero }}$ approaching $\lambda_{\text {pole }}$ as the hole size vanishes.

An alternative derivation (Genet et al., 2003) closely followed the original one by Fano (1961). The final expression coincides with Eq. (22), but it is written in a form that further clarifies the physical meaning of the different quantities involved:

$$
T(\lambda)=T_{\mathrm{bg}}(\epsilon+q)^{2} /(1+\epsilon)^{2}
$$

with

$$
\epsilon=\left[\omega-\left(\omega_{\mathrm{SM}}+\Delta\right)\right] /(\Gamma / 2), \quad q=2 \delta / \Gamma,
$$

where $\omega_{\mathrm{SM}}$ is the frequency of the unperturbed surface EM mode involved in the process, $\delta$ is the ratio between the transition amplitudes of the incident wave into the resonant state and the direct (background) channel, and $\Delta$ and $\Gamma$ are the frequency width and the frequency shift of the resonant state due to coupling to the continuum, respectively.

A generalization to the case in which several surface modes contribute appreciably to the transmission in a given spectral window was given by Fan et al. (2005) and Chang et al. (2005):

$$
T(\lambda)-T_{\mathrm{bg}}=T_{a} \frac{\left(1+\sum_{r} q_{r} / \epsilon_{r}\right)^{2}}{\left(1+\sum_{r} \epsilon_{r}^{-1}\right)^{2}},
$$

where $T_{a}$ is a parameter reflecting the strength of the resonant process and $q_{r}$ and $\epsilon_{r}$ are trivial extensions of the expressions for $q$ and $\epsilon$ given above, for the $r$ th surface mode this time. Usually these theoretical parameters are phenomenologically adjusted to match the computed transmission spectra with the measured ones.

\section{Coupled-mode analysis}

Note that, despite the apparent geometrical simplicity of an infinitely periodic array of apertures in a metal 


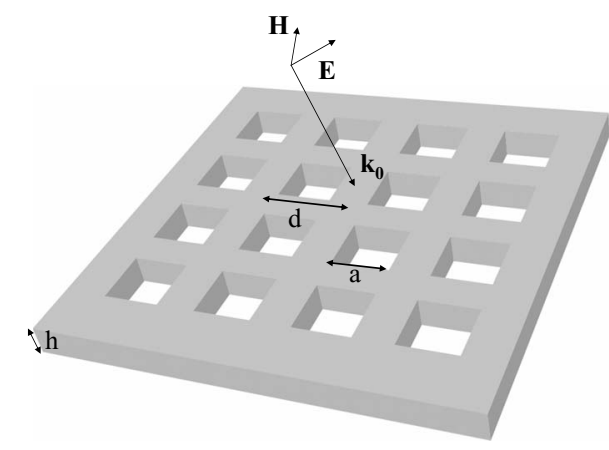

FIG. 24. Schematic of a 2D hole array of square holes of side $a$ forming a square array of period $d$ perforated through a metallic film of thickness $h$. The incident EM field is a $p$-polarized plane wave.

film, the system is still characterized by a large number of geometrical and material parameters. This, in turn, leads to the existence of different transmission regimes and the possible coexistence of several mechanisms. In keeping with previous sections, we use the theoretical formalism described in Sec. I.B and start by considering the metal as a PEC. Results obtained within this approximation are applicable to different frequency regimes by a simple scaling of all lengths defining the problem by a common factor. This simplified model shows the main physics and provides a framework for understanding the results obtained within more accurate numerical frameworks. Deviations from these results in the optical regime (caused by a finite dielectric function in the metal) will be discussed later. As a paradigmatic case, we consider the holes to have a square cross section merely because then all quantities involved in the formalism have simple analytical expressions. Figure 24 shows a schematic of the structure under study: an infinitely periodic square array with period $d$ of square holes of side $a$ perforated through a free-standing metal film of thickness $h$.

Figure 25 shows the normal incidence transmittance spectra for a square lattice of holes in a PEC film. The cutoff wavelength for the fundamental waveguide mode in the hole is $\lambda_{C}=2 a$, so for $\lambda>0.8 d$ the waveguide modes within the hole are evanescent. The solid curve in Fig. 25 is the full multimode result, obtained using the method described in Sec. I.B. The dashed line in Fig. 25 is the transmission spectrum computed within the singlemode approximation [set of Eqs. (3)], in which only the $\mathrm{TE}_{01}$ waveguide mode is considered in the modal expansion within each hole. In this case, the EM coupling between holes forming the array is accounted for by the propagator $G$ that can be expressed as a sum over both diffracted modes and the two polarizations ( $s$ and $p$ ): $G=\sum_{\vec{k} \sigma} Y_{\vec{k} \sigma}\left|\left\langle\mathrm{TE}_{01} \mid \vec{k} \sigma\right\rangle\right|^{2}$. In the previous expression, the sum runs over all wave vectors of the form $\vec{k}=\vec{k}_{0}+\vec{k}_{R}$, with $\vec{k}_{0}$ the parallel component of the incident wave vector and $\vec{k}_{R}$ a wave vector of the $2 \mathrm{D}$ reciprocal lattice.

When evaluating the results for the dashed curve in Fig. 25, as many diffraction modes as needed for conver-

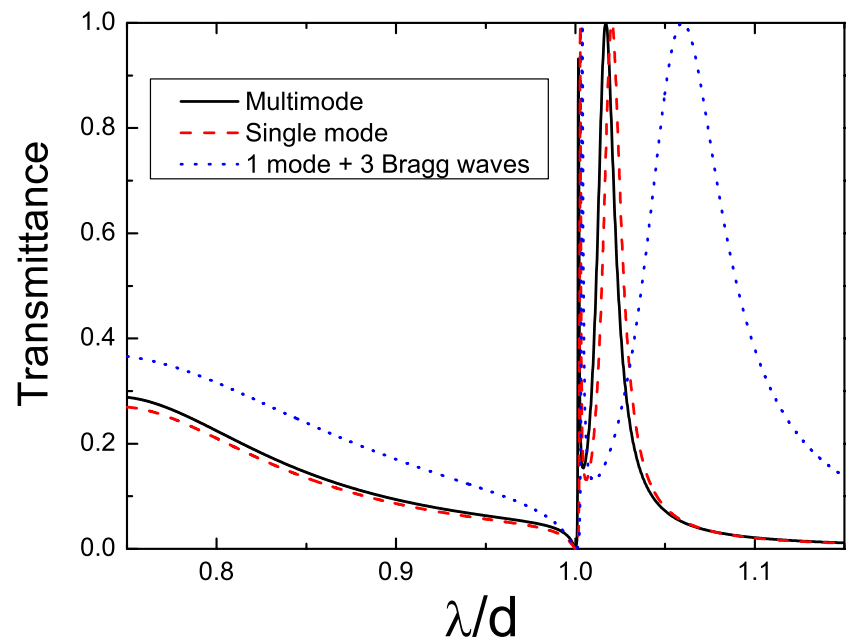

FIG. 25. (Color online) Transmittance through a hole array drilled in a PEC film. The side of the hole is $a=0.4 d$ and the film thickness is $h=0.2 d$. The solid curve is the result from the converged multimode expansion. The dashed curve is for the single-mode expansion discussed in this work. The dotted curve corresponds to the minimal model (see text).

gence were included in the sum defining G. As Fig. 25 shows, the single-mode approximation (SMA) provides a good estimation of the exact transmission curve even when the condition $a / \lambda \ll 1$ is not satisfied (in the considered case $a / \lambda \sim 0.3-0.5)$. The validity of the SMA arises from the stronger coupling of the relevant diffraction modes with the fundamental waveguide mode than with higher modes (which present a more rapid spatial variation). This mechanism takes precedence over the faster decay of higher-order waveguide modes inside the hole, thus explaining why the SMA is still a good approximation even for relatively thin films (standard EOT studies in the optical regime are usually done for $h / d$ $\approx 0.3-0.5$ ). The dotted curve in Fig. 25 was also computed within the SMA, but instead of summing as many diffraction orders in the sum defining $G$ as needed to achieve convergence, only the terms with $p$ polarization and reciprocal-lattice vectors $\vec{k}_{R}=0, \pm 2 \pi / d \vec{u}_{x}$ were considered [this is the so-called minimal model as described by Martin-Moreno et al. (2001)]. While consideration of additional diffraction modes modifies the final numerical result, the basics of EOT are already present in this extremely simplified model. This conclusion that the resonant transmission process is mainly governed by a dominant mode within the holes was corroborated by Enoch et al. (2002). By artificially doubling the imaginary part of the propagation constants of the calculated Bloch modes of the holey film, they concluded that the transmission was dictated by just one of those Bloch modes.

Three features are readily visible in Fig. 25: one very deep minimum at $\lambda=d$ and two transmission peaks. The presence of two peaks is associated with the EM coupling via the holes between the two interfaces of the holey metal film, which we discuss later. The deep minimum, in a PEC, is related to a divergence of $G$. Recall that $G$ diverges whenever a $p$-polarized diffraction 


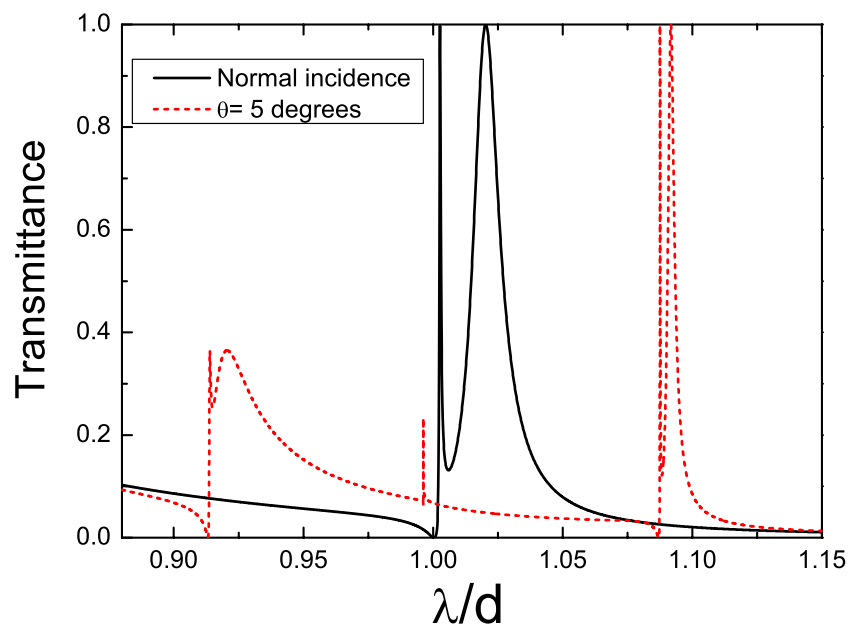

FIG. 26. (Color online) Transmittance for a $p$-polarized plane wave impinging into a hole array within the single-mode and PEC approximations. The side of the hole is $a=0.4 d$ and the film thickness is $h=0.2 d$. The solid curve is for normal incidence, while the dashed curve is for incidence at $\theta=5^{\circ}$.

mode has $k_{z}=0$, since the admittance of a $p$-polarized plane wave is defined as $Y_{\vec{k} p}=k_{\omega} / k_{z}$. In this case, Eqs. (4) give $E=E^{\prime}=0$, so there is no transmission because no field penetrates into the holes. Consider first the particular case of normal incidence. Then the diffraction order characterized by the reciprocal-lattice vector $\vec{k}_{R}$ has $k_{z}$ $=0$ for $\left|\vec{k}_{R}\right|=k_{\omega}$. Therefore, for a square lattice and with the metal treated as a PEC, transmission minima occur at $\lambda_{n, m}^{\min }=d / \sqrt{n^{2}+m^{2}}$, where $n$ and $m$ are integers, and the largest wavelength at which a deep minimum occurs is $\lambda_{ \pm 1,0}^{\min }=\lambda_{0, \pm 1}^{\min }=d$. The spectral position of the transmission features changes with the angle of incidence. Consider the case in which the incident wave vector forms an angle $\theta$ with the surface normal, and its parallel component lies along one of the axes of the square array. Then the condition of grazing diffraction occurs at $\left|k_{\omega} \sin \theta \vec{u}_{x}+\vec{k}_{R}\right|=k_{\omega}$. Therefore, at $\theta \neq 0$, the minima associated with $\lambda_{ \pm 1,0}^{\min }$ split, appearing now at $\lambda_{ \pm 1,0}^{\min }$ $=(1 \pm \sin \theta) d$ and $\lambda_{0, \pm 1}^{\min }=\sqrt{1-\sin ^{2} \theta} d$. Figure 26 shows such splitting between transmission minima for a hole array with the same geometrical parameters as in Fig. 25 but illuminated with a $p$-polarized plane wave impinging at $\theta=5^{\circ}$ : the expected minima are seen at wavelengths $\lambda_{-1,0}^{\min }=0.913 d, \lambda_{0, \pm 1}^{\min }=0.996 d$, and $\lambda_{1,0}^{\min }=1.087 d$.

We now give support to the statement that the transmission peaks are due to the presence of leaky surface EM modes of the corrugated metal film. For this, it is convenient to work within the multiple-scattering formalism. Within the single-mode approximation, the zero-order transmission coefficient can be expressed as a function of two-media scattering coefficients:

$$
t_{0}=\tau^{12} e^{i q_{z} h} \tau^{23} /\left(1-e^{2 i q_{z} h} \rho^{2}\right) .
$$

As for the case of slit arrays presented in Sec. III.A.2, the appearance of transmission peaks can be related to resonant properties of $\rho$, which gives the reflection coef-

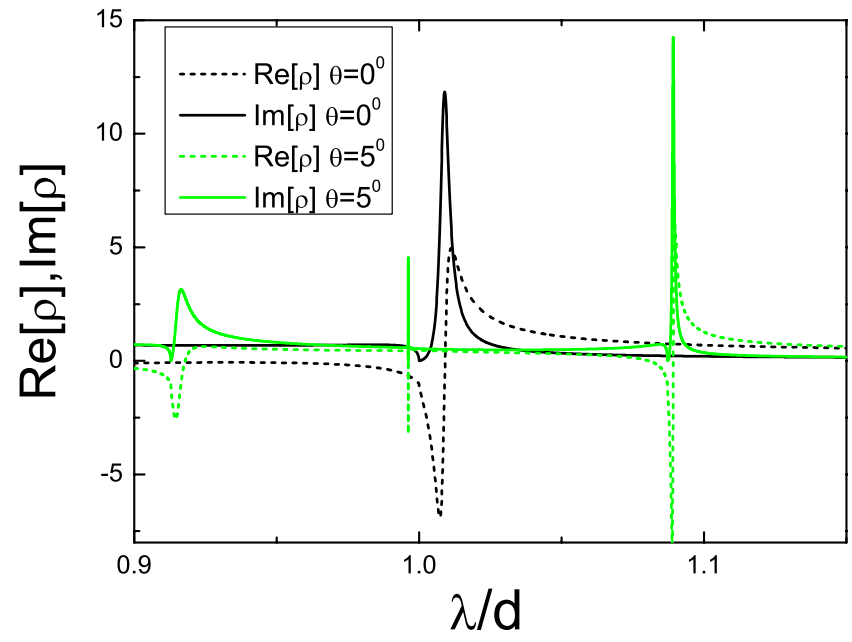

FIG. 27. (Color online) Real (discontinuous lines) and imaginary (continuous lines) parts of $\rho$, the reflection amplitude for a Bloch combination of $\mathrm{TE}_{01}$ waveguide modes impinging into vacuum. The Bloch wave vector forms either $0^{\circ}$ with the normal to the surface or $5^{\circ}$. The side of the hole is $a=0.4 d$.

ficient for EM fields coming from the interior of the hole. Figure 27 shows the real and imaginary parts of $\rho$ for the cases considered in Fig. 26. Large values for $\operatorname{Im}(\rho)$ and anomalous behavior for $\operatorname{Re}(\rho)$ occur at spectral positions close to the transmission resonances for both $\theta=0^{\circ}$ and $5^{\circ}$. The spectral dependence of $\operatorname{Re}(\rho)$ and $\operatorname{Im}(\rho)$ is the one expected, through Kramers-Kronig relations, for causal functions close to localized resonances (Landau et al., 1960) (a reflected field requires a preexisting incident one, so $\rho$ must satisfy causality). Recall that $\rho$ is a two-media scattering coefficient, so its resonances mark the existence of surface EM modes bound to a single interface.

Figure 27 shows spectral regions with $|\rho|>1$, implying that the reflected field has an amplitude that is larger than the incident one. This is counterintuitive as it seems to indicate that the reflected current is larger than the incident one. However, this apparent paradox is solved because, for evanescent modes, energy conservation and causality only dictate $\operatorname{Im}(\rho)>0$, saying nothing about $|\rho|$. In what follows, we demonstrate how the existence of a resonant behavior of $\rho$ is a fingerprint of the emergence of geometrically induced surface EM modes in a semiinfinite holey PEC film.

In principle, the band structure of these surface EM modes could be obtained by finding the solutions of $G$ $-\Sigma=0$ as a function of the incident wave vector $\vec{k}_{0}$ as we did previously for the case of $1 \mathrm{D}$ arrays of slits. Note that, as we are now considering a semi-infinite perforated metal, $G^{V}=0$ in Eqs. (3). Complex values of $\vec{k}_{0}$ must be considered in the search for leaky modes, while truly bound surface modes can show up only for evanescent incident wave fields (with modulus of the parallel momentum $\left|\vec{k}_{0}\right|$ larger than $\left.k_{\omega}\right)$. However, the overall form of the band structure can be obtained without the need for numerical computations. For this, it is conve- 
nient to consider that the metal has an arbitrarily large (but not infinite) negative dielectric constant. In this case, a flat metal surface supports truly bound SPPs. The presence of a periodic array of small holes can be considered within a perturbative approach. The result is that the dispersion relation of surface EM modes in the corrugated structure will closely follow the one for SPPs, except for $\vec{k}_{0}$ values lying close to a Brillouin zone boundary, where bands bend in order to accommodate band gaps. For frequencies above the first band gap, surface modes couple to radiation, thus becoming leaky. However, the band sector below the first band gap represents a truly bound surface mode. As a result of the band bending caused by the periodic array of holes, the lowest band of surface modes separates from the light line, therefore binding the EM field more strongly to the surface. This line of reasoning has been presented before (Ulrich, 1974) and is the center of the field of frequency-selective surfaces (Munk, 2000). Note that this description of the dispersion relation is based on general arguments, applicable not only to SPPs but also to any type of wave in periodic media.

In the particular case of the EM field in a perforated metal surface, there is an additional mechanism for periodicity-induced binding of the EM fields to the surface. In order to illustrate this point, we calculate the bands of a holey metal surface within the PEC approximation. Note that PEC flat surfaces do not support bound SPPs. In a first approximation, we consider the hole array as a metamaterial and compute the band structure of surface EM modes by matching the average EM fields over the surface; the effect of periodicity will be included on a second stage. Average fields can be readily matched by neglect of diffraction effects, as done previously for the case of slit arrays (see Sec. III.A.2). In this way, all information about the underlying lattice is lost.

Figure 28 shows the dispersion relation along the $\Gamma-X$ direction for surface EM modes bound to an isolated holey interface for both $\epsilon_{\text {hole }}=1$ (solid curve) and $\epsilon_{\text {hole }}$ $=9$ (dotted curve). These two curves have been obtained by means of Eq. (A27). Note that bands flatten at certain frequencies, although no diffraction effects have been included yet. This result can be expressed in the language of metamaterials by assigning an effective dielectric permittivity $\left(\epsilon_{\mathrm{eff}}\right)$ and an effective magnetic permeability $\left(\mu_{\text {eff }}\right)$ to the structured surface. This derivation was first done by Pendry et al. (2004) and can also be found in Sec. A.5, resulting in $\mu_{\mathrm{eff}}=8 a^{2} / \pi^{2} d^{2}$ and $\epsilon_{\mathrm{eff}}$ given by

$$
\epsilon_{\mathrm{eff}}(\omega)=\left(\pi^{2} d^{2} \epsilon_{\text {hole }} / 8 a^{2}\right)\left(1-\omega_{p}^{2} / \omega^{2}\right) .
$$

This functional form for $\epsilon_{\mathrm{eff}}(\omega)$ is similar to Drude's expression for the dielectric constant of a metal. Therefore, it can be said that a corrugated PEC surface approximates the flat surface of a real conductor, characterized by a (geometry-dependent) "effective plasma frequency" $\omega_{p}=\left(c / \sqrt{\epsilon_{\mathrm{hole}}}\right) \pi / a$, which coincides with the cutoff frequency of the hole. Actually, the system is an-

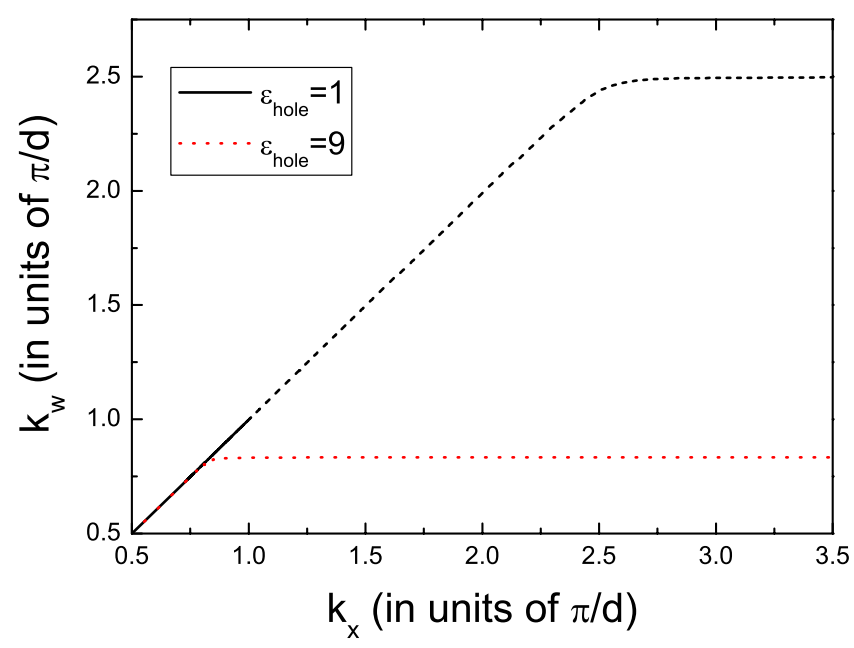

FIG. 28. (Color online) Approximate dispersion relations for geometrically induced SPPs in a semi-infinite holey PEC film computed without diffraction effects. The side of the square holes is $a=0.4 d$. A dielectric medium with dielectric constant $\epsilon=1$ (solid curve) or $\epsilon=9$ (dotted curve) fills the holes. The discontinuous line marks the spectral region of what will become leaky modes when diffraction effects are considered.

isotropic, so care must be taken about the different components of the effective dielectric constant tensor (Pendry et al., 2004). The anisotropy is also responsible for the fact that the flat region of the dispersion curve for the geometrically induced SPP appears at $\epsilon_{\text {eff }}=0$, whereas the dispersion relation for truly SPPs bound to the interface between two isotropic media flattens at $\epsilon=-1$.

Periodicity has two effects on the dispersion relation of the geometrically induced surface EM mode: it opens gaps at wave vectors $\vec{k}$ close to Brillouin zone boundaries and couples bands with $|\vec{k}|>\pi / d$ to radiative modes. This is shown in Fig. 28, where the discontinuous lines represent modes that become leaky when diffraction effects are considered. Surface EM modes still appear if higher-order waveguide and diffraction modes are included in the calculation, and their dispersion relation still flattens at $\omega=\omega_{p}$, given by the hole cutoff. However, the dispersion relation moves closer to the light line and strong confinement occurs only for frequencies much closer to $\omega_{p}$ than predicted by the effective parameter expression [Eq. (27)] (De Abajo and Saenz, 2005; Hendry, Hibbins, and Sambles, 2008).

Experimental verification of the existence of surface EM modes in perforated PEC films has been reported by different groups. Using two prisms to couple microwave radiation to and from surface EM modes in extremely thin perforated metal films, Ulrich and Tacke (1973) measured the dispersion relation of those modes in the regime in which the departure from the light line comes from band bending due to diffraction effects (see the discussion on the two mechanisms for band bending presented previously). Experiments showing band bending associated with the cutoff frequency of the hole waveguide were reported by Hibbins et al. (2005) who 


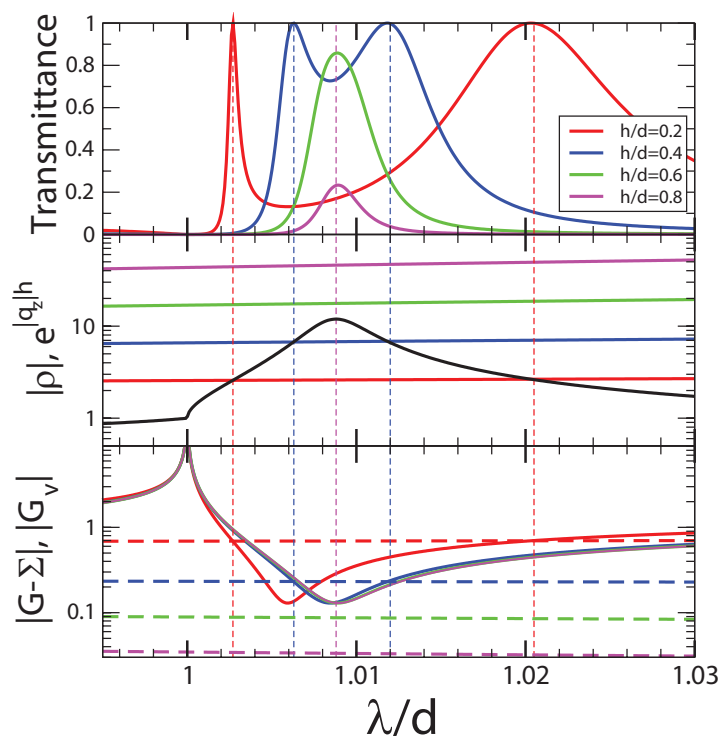

FIG. 29. (Color) Evolution of the transmission with the thickness of the metal film. Top panel: Normal-incidence transmittance spectra through a hole array in a PEC film for different metal thicknesses $h$. The side of the holes is fixed at $a=0.4 d$. Middle panel: Spectral dependence of $|\rho|$ (black curve) and $e^{\left|q_{z}\right| h}$ (color lines for the corresponding metal thicknesses represented in the upper panel). Bottom panel: Spectral dependence of $|G-\Sigma|$ (continuous line) and $\left|G^{V}\right|$ (dashed lines). The vertical discontinuous lines are guides for the eye marking the crossings of $|\rho|$ with the different $e^{\left|q_{z}\right| h}$ curves or, alternatively, the cuts between $|G-\Sigma|$ and $\left|G^{V}\right|$.

analyzed the angular dependence of the transmission peaks in 2D arrays of holes infiltrated with wax. These experiments were also carried out in the microwave regime of the EM spectrum. In a more recent development, the subwavelength confinement associated with the existence of spoof SPPs in 2D arrays of dimples was experimentally tested in the terahertz range (Williams et al., 2008).

Once we have explained the physical origin of the resonant features of $\rho$, which contains information about only one surface, the questions are how the geometrically induced surface EM modes couple in a metal film of finite thickness and how this coupling affects the transmission spectrum. The answers to these questions are shown in Fig. 29. The top panel shows the transmittance spectra for different metal thicknesses, while the middle panel renders the corresponding spectral dependence for both $e^{\left|q_{z}\right| h}$ (which in the subwavelength regime is always larger than 1 and increases exponentially with $h$ ) and $|\rho|$ (which, being a two-media scattering coefficient, does not depend on metal thickness).

As Fig. 29 shows, transmittance maxima occur at the wavelengths of minimal distance between the $|\rho|$ and $e^{\left|q_{z}\right| h}$ curves [see the denominator of Eq. (26)]. Depending on the metal thickness, two transmission regimes appear for any given resonance of $|\rho|$. For small metal thicknesses (but still larger than three to four times the skin depth, so the metal is optically opaque and the considered model makes sense), the curves for $|\rho|$ and $e^{\left|q_{z}\right| h}$
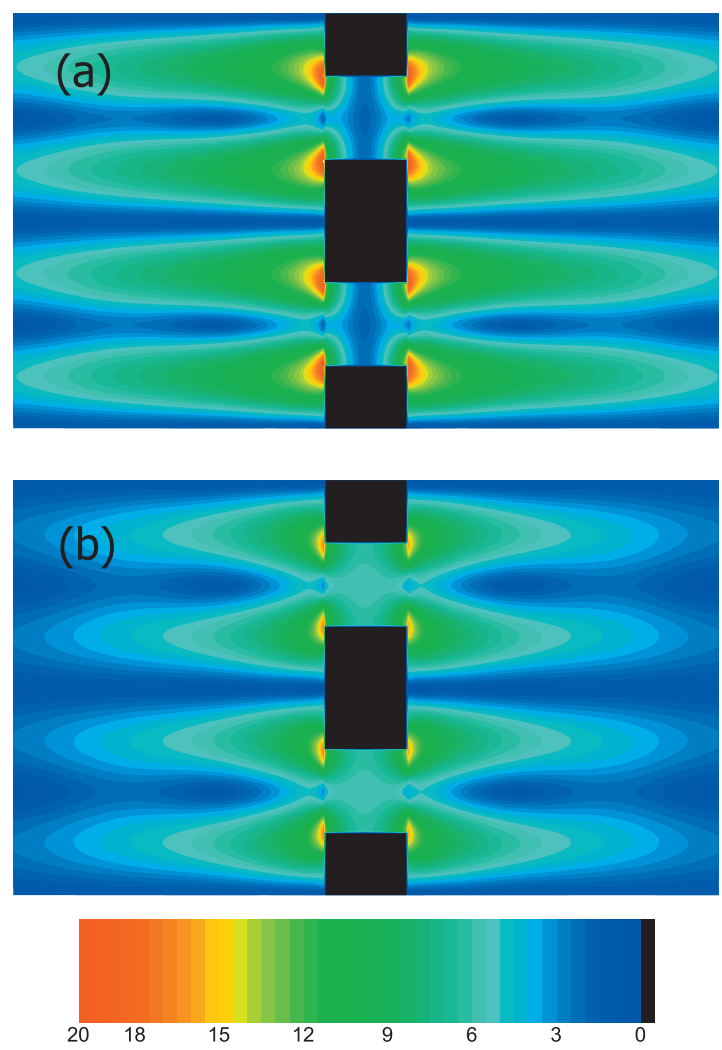

FIG. 30. (Color) $E$-field amplitude evaluated at the two resonant wavelengths in the $x-z$ plane that cuts the square holes by their centers for the case $h=0.4 d$ depicted in Fig. 29. (a) The lower wavelength (antisymmetric mode). (b) The amplitude for the peak appearing at a longer wavelength (symmetric mode).

cross twice, leading to the presence of two transmission maxima which, for a lossless metal, reach $100 \%$ transmission (Martin-Moreno et al., 2001). For thick enough films, $e^{\left|q_{z}\right| h}$ is larger than the maximum value of $|\rho|$. In this case, the curves for $|\rho|$ and $e^{\left|q_{z}\right| h}$ do not cross but there is still one transmission maximum at the wavelength of minimum distance between them, i.e., approximately at the spectral position of the maximum of $|\rho|$. Note that a continuous transition from a transmission spectrum with two EOT peaks to one with a single attenuated maximum also occurs if the thickness of the film is fixed but the size of the hole is gradually reduced.

The previous discussion was elaborated within the multiple-scattering formalism. However, the analysis of the existence of coupled leaky modes and their relation to transmission maxima can also be done within the formalism involving $E$ and $E^{\prime}$, which has been used throughout this review. In this formulation, the condition for resonant field enhancement, and correspondingly resonant transmission, can be obtained from the set of Eqs. (3) as $|G-\Sigma|=\left|G^{V}\right|$. The bottom panel of Fig. 29 shows the spectral dependence of both $|G-\Sigma|$ and $\left|G^{V}\right|$ for different metal thicknesses considered in the top and middle panels. As Fig. 29 shows, there is a univocal correspondence between the spectral positions of the transmission maxima and the wavelengths of minimum distance between $|G-\Sigma|$ and $\left|G^{V}\right|$. Note that 
the resonances appear close to the divergences of $G$, associated with the condition $k_{z}=0$ which marks the spectral location of the transmission minima.

The existence of these two regimes (two hightransmission peaks or one exponentially attenuated resonance) can be understood in terms of typical times or distances in the system as follows. As previously stated, a peak in $\operatorname{Im}(\rho)$ marks the existence of a leaky surface EM mode bound to a single interface. In a film there are two such modes (one for each interface) that interact through the evanescent fields inside the hole. This results in coupled EM modes that are the symmetric and antisymmetric combinations of the original surface modes (see Fig. 30) in much the same way as electronic states of isolated atoms couple to form molecular levels. These modes are, in general, leaky, i.e., they couple to radiation modes. Nevertheless, additional insight can be gained if we compute their frequencies, neglecting the coupling into radiative modes. In this case, the frequency difference between the two modes is inversely proportional to the time $t_{\text {res }}$ that the EM field has to stay in the system in order to determine that there are two resonant levels. Note that this time depends exponentially on the metal thickness. During time $t_{\text {res }}$, the EM field travels a distance $L_{\text {res }}$ on the surface which, assuming that the field travels at speeds close to that of light, is $L_{\text {res }} \approx c t_{\text {res }}$. The effect of the coupling to radiative modes can be considered through the width $\Delta \lambda$ of the resonance in $\operatorname{Im}(\rho)$, which is related to the time that the EM field stays at the surface before being radiated $t_{\mathrm{rad}}$, or equivalently to the distance that the EM field can travel during that time $L_{\text {rad }}$. Using the relations $\nu=c / \lambda$ and $\Delta \nu \times \Delta t \sim 1$, and assuming that the EM field travels at the surface at almost the speed of light, we obtain $L_{\mathrm{rad}} \approx c t_{\mathrm{rad}} \sim \lambda^{2} / \Delta \lambda$. Therefore, for the particular case of resonances appearing at wavelengths close to the period, the number of periods that the EM wave travels before it is radiated is roughly $\Delta \lambda / d$. If the geometrical parameters defining the array are such that $t_{\text {rad }}>t_{\text {res }}$, the EM field stays in the film long enough to sense two different resonant levels and the transmission is high at the two corresponding frequencies. In the opposite situation $\left(t_{\text {rad }}<t_{\text {res }}\right)$, the EM field is radiated before it can differentiate between two resonances. Moreover, if $t_{\text {rad }} \ll t_{\text {res }}$ the process is more like (coherent) sequential tunneling, where the incoming field first gets trapped in a surface mode, tunnels to the surface mode at the other interface, and finally exits via outgoing radiative modes. The transmittance is enhanced in this case as the photon can use two intermediate resonant states (the surface EM mode at each interface) to cross the metal film, but the enhancement is not as efficient as when the EM field hops back and forth several times building up a constructive interference. The previous discussion of $t_{\mathrm{rad}}$ was made on the basis of the properties of one interface and does not consider that the symmetric and antisymmetric combinations of surface modes couple differently to radiation modes. In fact, EM fields radiated from each surface in the antisymmetric combination partially cancel

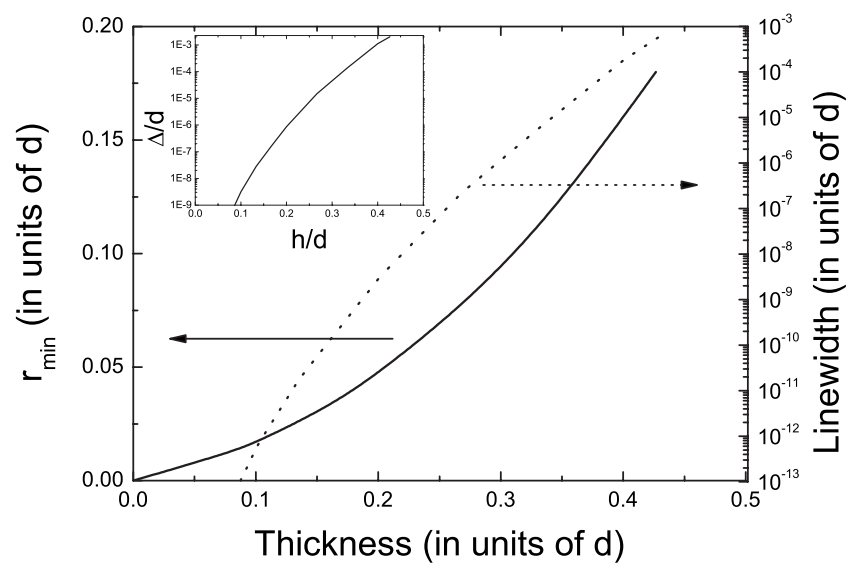

FIG. 31. Evolution as a function of metal thickness $(h)$ of the threshold value for the radii of circular holes $\left(r_{\min }\right)$ forming a $2 \mathrm{D}$ array in order to have $100 \%$ transmission at, at least, one resonant wavelength $\lambda_{R}$ (full line). The dashed line represents the linewidth associated with the transmission peak in hole arrays with $r=r_{\min }$ for each value of $h$. The inset shows the spectral separation between $\lambda_{R}$ and the period of the array $d$, $\Delta=\lambda_{R}-d$, evaluated at $r=r_{\min }$ for each value of $h$.

each other. The smaller coupling to radiative modes in the antisymmetric combination of surface modes than to that in the symmetric one translates into a larger $t_{\mathrm{rad}}$ and, correspondingly, a narrower peak. This can be seen in, for instance, Fig. 29.

The transition from the regime in which the transmittance spectrum presents two peaks reaching $100 \%$ values to that of one exponentially attenuated peak is characterized in Fig. 31. Figure 31 shows the dependence with metal thickness of $r_{\text {min }}$, defined as the minimum hole radius such that the transmittance is complete at, at least, one resonant wavelength $\lambda_{R}$. Calculations were performed with the coupled-mode formalism. The continuous curve divides the parameter space (radius of the hole $r$ and metal thickness $h$ ) in two regions. Within the region at the left of the curve, the transmittance spectrum has at least one resonant peak reaching complete transmission while the transmittance in hole arrays pertaining to the region at the right presents a single resonant peak with maximum less than $100 \%$. De Abajo et al. (2005) found that light transmission through perforated PEC films with negligible thickness is complete at some resonant wavelength, even for arbitrarily small holes. This is reflected in Fig. 31 in that $r_{\min } \rightarrow 0$ when $h \rightarrow 0$. The dependence of $\lambda_{R}$ with $h$ is described in the inset of Fig. 31. As $h$ decreases, $\lambda_{R}$ rapidly approaches the lattice parameter $d$ (notice the logarithmic scale for $\Delta$ ). Figure 31 also shows how the linewidth of the transmission peak at the minimum radius $r_{\text {min }}$ evolves with film thickness. When expressed in units of the period, this linewidth is inversely proportional to the number of periods needed in the hole array such that the holes at the center behave as in an infinite system. The fast decrease of the linewidth with film thickness (notice again the logarithmic scale) implies that finite-size effects are increasingly important for smaller holes. 


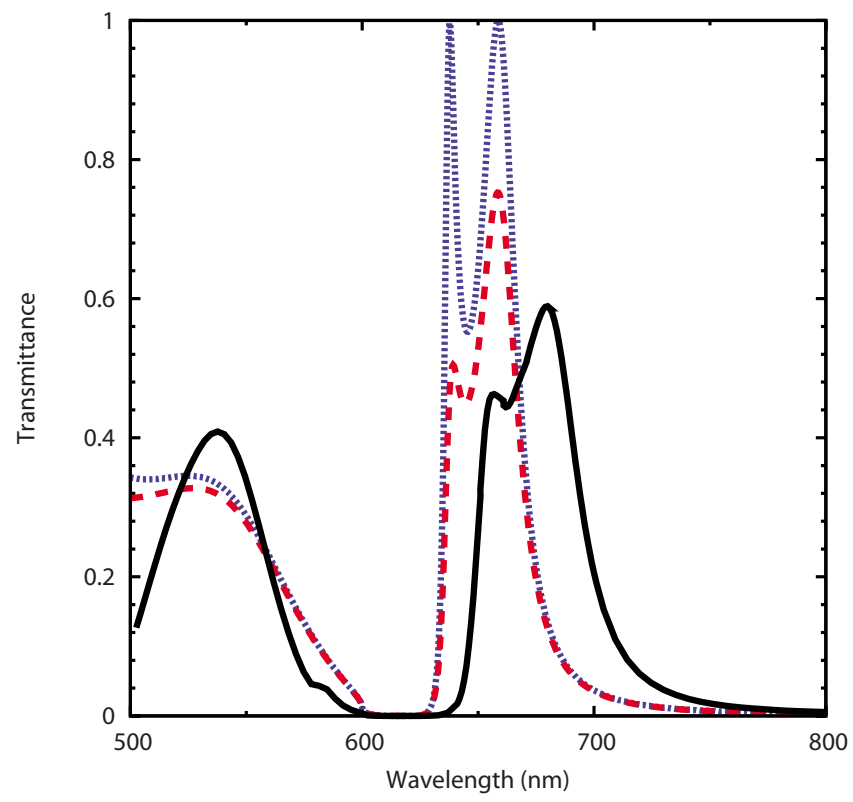

FIG. 32. (Color online) Transmittance spectra through an array of circular holes in a free-standing silver film of thickness $h=275 \mathrm{~nm}$. The radius of the hole is $r=134 \mathrm{~nm}$ and the period of the array is $d=600 \mathrm{~nm}$. The dielectric function of silver is taken from Palik, 1985. The solid curve is the FDTD result, obtained with a discretization mesh of $2 \mathrm{~nm}$. The dashed curve is the result obtained within the coupled-mode method and the SIBC approximation. The dotted curve is for the last case, but with the absorption in the metal set zero, i.e., $\operatorname{Im}\left(\epsilon_{M}\right)=0$.

The previous analysis was for infinitely periodic arrays in a lossless media. Beyond this idealization, consideration of absorption and finite arrays introduces two additional time scales: the typical time the EM field stays at the surface before being absorbed $\left(t_{\mathrm{abs}}\right)$ and the time needed for crossing the array $\left(t_{L} \approx L / c\right.$, where $L$ is the length of the array). Several transmission regimes appear depending on the relation of these four time scales $\left(t_{\mathrm{res}}, t_{\mathrm{rad}}, t_{\mathrm{abs}}\right.$, and $\left.t_{L}\right)$, which may affect the different transmission resonances differently. A detailed analysis of both finite-size effects and type of metal will be considered later on in this review. We mention here that the EOT narrow peak related to the antisymmetric combination of surface modes is more sensitive to both absorption and finite-size effects than the one involving the symmetric mode (which appears at longer wavelengths). Actually, in the experiments, only the latter is clearly differentiated. Nevertheless, experiments have shown the existence of two regimes in the evolution of the main EOT peak(s) with thickness (Degiron et al., 2002), as theory predicts.

The effect of both a finite dielectric function and the inherent absorption in a real metal at optical frequencies is shown in Fig. 32. This figure shows the computed transmission spectra obtained with different approximations for an infinite array of circular holes in a silver film for a representative set of geometrical parameters. The calculated cutoff wavelength of the hole waveguide is in this case $590 \mathrm{~nm}$. The FDTD result is obtained using a small discretization mesh $(2 \mathrm{~nm})$ and can be considered as virtually exact. Figure 32 shows that transmittance peaks reaching $100 \%$ are still expected for a lossless metal and also that absorption prevents $100 \%$ transmission, having a larger effect on the narrower peaks, as previously discussed.

Another feature that is evident from Fig. 32 is that the transmission minimum at $\lambda_{10}^{\min }$ appears redshifted in a real metal film with respect to the PEC case which would have $\lambda_{10}^{\mathrm{min}}=600 \mathrm{~nm}$. In the present formalism, this minimum is still due to the divergence of $G$ which, in a real metal, occurs when the corresponding diffraction order has a $k_{z}$ satisfying $k_{z}+Z_{S} k_{\omega}=0$. As mentioned, this is exactly the condition for the existence of SPPs with parallel momentum, $k_{\|}=2 \pi / d$, within the SIBC approximation in an uncorrugated metal surface. Therefore, in general, transmission minima are linked to the dispersion relation of SPPs for the flat interface, thus appearing at $\lambda_{n, m}^{\min }=d \sqrt{\epsilon_{M} /\left(\epsilon_{M}+1\right)} / \sqrt{n^{2}+m^{2}}$.

\section{Dependence on the material properties}

The role of the type of metal is important in the extraordinary transmission and has been verified experimentally in several studies. The original observation was made on silver, gold, and chromium films at optical frequencies (Ebbesen et al., 1998). In keeping with the previous theoretical discussion, the intensity of the transmission peak decreases and its linewidth increases as the absorption, which is related to the imaginary part of the permittivity of the metal, increases (Grupp et al., 2000; Przybilla, Degiron, et al., 2006; Williams and Coe, 2006; Chang, Chuang, et al., 2007; Ctitis et al., 2007; Ekinci et al., 2007).

Exhaustive studies of the dependence of EOT peaks on the type of metal have been conducted both experimentally (Przybilla, Degiron, et al., 2006) and theoretically (Rodrigo et al., 2008). Figure 33(a) shows the evolution of the experimental EOT peak heights for different metals as a function of the resonant wavelength. Figure 33(b) also shows the FDTD calculated transmission spectra for $\mathrm{Ag}, \mathrm{Al}, \mathrm{Ni}$, and $\mathrm{W}$. The common conclusion of these two studies is that the two main parameters that characterize the influence of the dielectric constant of the metal on EOT peaks are the skin depth (which effectively enlarges the hole size, increasing the transmittance) and the absorption length (small absorption lengths impair the resonant process). "Good metals" such as $\mathrm{Au}, \mathrm{Ag}$, and $\mathrm{Cu}$ sustain EOT peaks with transmission values that may exceed even those of a PEC with the same geometrical parameters due to the penetration of the fields inside the metal. On the contrary, metals presenting large absorption, such as $\mathrm{Ni}$ and $\mathrm{Cr}$, display very low EOT peaks. The skin depth in $\mathrm{Al}$ is much smaller than in any other metal: correspondingly, EOT peaks in holey aluminum films resemble those in a PEC, except at the longer wavelengths in the optical regime, when absorption in $\mathrm{Al}$ increases and the EOT is more likely that corresponding to a "bad metal." The case of $\mathrm{W}$ is interesting, as in the optical regime it be- 

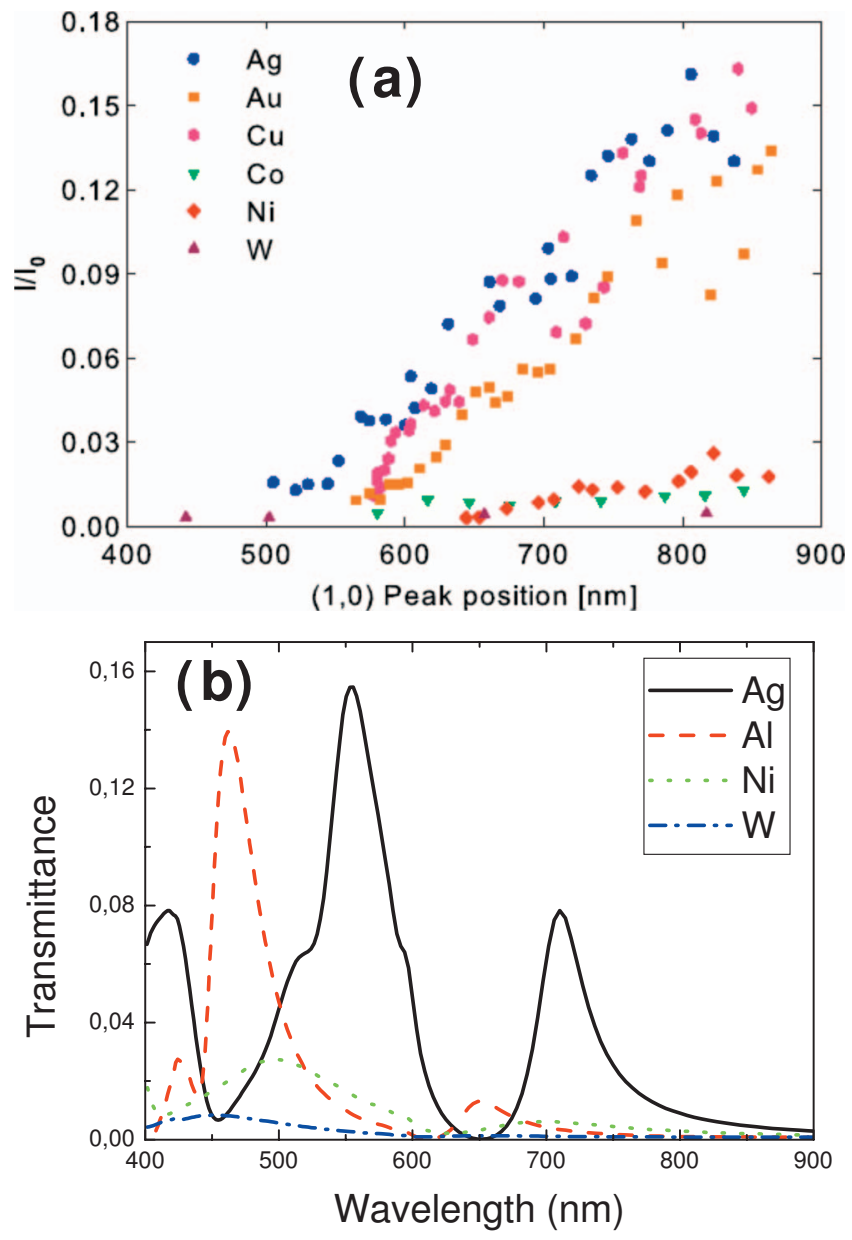

FIG. 33. (Color) Dependence of EOT with the material properties. (a) Experimental transmission intensities as a function of the $(1,0)$ transmission peak position for different metals. The samples consist of perforated metal slabs (thickness $250 \mathrm{~nm}$ ) deposited on a glass substrate. The period of the square array is varied between 200 and $500 \mathrm{~nm}$ and the ratio between the period of the array and the diameter of the holes is fixed at 1.75. (b) Calculated transmission spectra for an array of circular holes in a metal film deposited on a glass substrate for several metals. Calculations were performed with the FDTD method. The geometrical parameters considered are period $d=400 \mathrm{~nm}$, metal thickness $h=250 \mathrm{~nm}$, and hole radius $r=114 \mathrm{~nm}$. From (a) Przybilla, Degiron, et al., 2006 and (b) Rodrigo et al., 2008.

haves as a lossy dielectric. Still surface EM modes (known as Zenneck waves) exist in lossy dielectrics, leading to transmission peaks (Sarrazin and Vigneron, 2003), as discussed before. However, transmission peaks in $\mathrm{W}$ are much weaker than even those associated with bad metals (see Fig. 33).

EOT has also been observed for metals at microwave (Beruete, Sorolla, et al., 2004) and terahertz (Miyamaru et al., 2003; Cao and Nahata, 2004a, 2004b; Qu and Grischkowsky, 2004) frequencies, as could be expected from the emergence of EOT in perforated PEC films. In an experimental study in the terahertz regime, Lee et al. (2005) analyzed the evolution of the height of the EOT peak with increasing values of the side of the square holes forming a periodic array drilled in an aluminum film. In agreement with the EOT picture provided in Sec. III.B.2, it was found that the peak transmission increases as the side of the hole is enlarged, reaching $100 \%$ for the case of maximum coverage considered. In the terahertz regime, the peak intensity increases as the ratio of the absolute value of the real to the imaginary part of the dielectric constant increases (Azad et al., 2006). So far, extraordinary transmission has been observed for periodic arrays in a wide variety of materials for various frequency regimes: highly doped Si (Gomez Rivas et al., 2003), metal-organic conducting polymers (Matsui et al., 2006), $\mathrm{VO}_{2}$ (Donev et al., 2006), metalcoated $\mathrm{VO}_{2}$ double layers (Suh et al., 2006), $\mathrm{SrTiO}_{3}$ (Miyamaru et al., 2006b), GaAs (Wasserman et al., 2007), gold-coated GaAs (Zhou et al., 2008), amorphous $\mathrm{SiO}_{2}$ (Chen, Hamam, et al., 2007), and SiC (Urzhumov et al., 2007).

Similarly, a thin layer with a low imaginary permittivity deposited on a more absorbing metal (Grupp et al., 2000) or semiconductor (Fang et al., 2007) is able to influence the transmission. As expected from the SPP dispersion relation, the transmission is also sensitive to the refractive index of the dielectric material at the metal interface which will affect both the peak position and the transmission intensity (Krishnan et al., 2001). As a consequence, the presence of even a thin dielectric layer can be detected (Tanaka et al., 2005) and used for sensing purposes (Brolo, Arctander, et al., 2004), as discussed in Sec. V.A. The fact that only a thin layer with a thickness of the order of the skin depth suffices to establish EOT has also been verified by coating thin metal layers on periodic arrays of microspheres (Landstrom et al., 2005, 2009; Farcau and Astilean, 2007).

\section{Size and shape dependence}

In the first report of EOT the size of the holes was already shown to have an important effect on the height of the transmission peaks (Ebbesen et al., 1998). This observation has been confirmed at different incident angles for various reciprocal-lattice directions (Williams et al., 2004). As the hole diameter is increased, both the intensity and the linewidth of the transmission peaks increase (Ishihara, Ikari, et al., 2005; Miyamaru et al., 2006a). These observations are consistent with a picture in which radiative damping of the SPP modes occurs due to scattering with the holes (Kim et al., 2003; Naweed et al., 2003). When a large range of hole sizes is investigated, it becomes clear that no single power-law scaling can describe the increase of the normalized transmission (van der Molen et al., 2004). Figure 34 shows the dependence on hole size of the normalized transmission for a large range of hole sizes. A strong dependence of the transmitted intensity on hole size can be observed for small sizes, where the normalized transmission scales as a high power of the diameter. At larger hole sizes and for a larger normalized transmission the trend saturates. The existence of two regimes as a function of the hole size can be understood with the help of the coupled- 


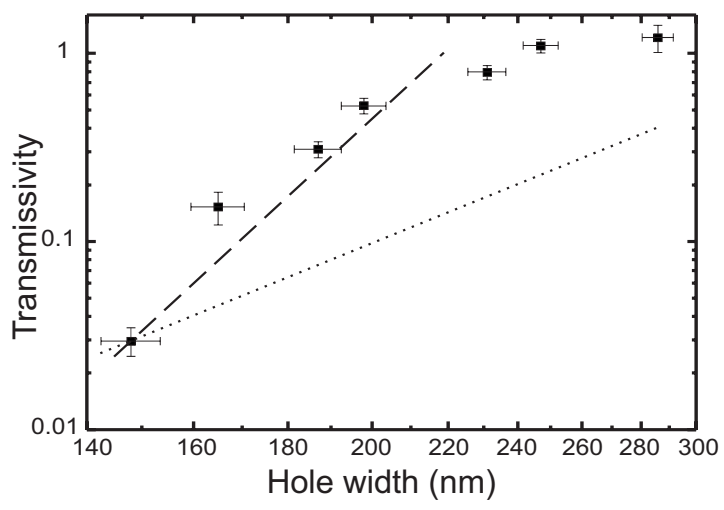

FIG. 34. Measured dependence of the transmission of the $(1,0)$ peak through an array of square holes as a function of size of the holes (double logarithmic representation). The thickness of the gold films is $200 \mathrm{~nm}$ and they are deposited on a glass substrate. All arrays investigated have a period of $425 \mathrm{~nm}$. The dotted line obeys $T \propto d^{4}$. The dashed line corresponds to $T$ $\propto d^{9}$. For smaller holes the increase in normalized transmission is easily faster than $d^{4}$, while for the larger holes the normalized transmission saturates. From van der Molen et al., 2004.

mode analysis presented in Sec. III.B.2. When the hole size is small, the factor $e^{\left|q_{z}\right| h}$ is large as the waveguide mode inside the hole decays rapidly. Then the field enhancement coming from the reflection coefficient $|\rho|$ cannot compensate for the exponentially small factor (mathematically, there is no cut between $e^{\left|q_{z}\right| h}$ and $|\rho|$ ), and the transmission is extremely low. As the hole size is increased, $e^{\left|q_{z}\right| h}$ is strongly reduced, leading to the emergence of a cut between $|\rho|$ and $e^{\left|q_{z}\right| h}$, i.e., the transmission reaches its maximum value and saturates.

Hole size also affects the spectral position of the transmission peaks. A small blueshift can be observed for the main peaks as the hole size is decreased (Naweed et al., 2003; van der Molen et al., 2004). In this last reference, it is reported that the main EOT peak shifts by $\Delta \lambda \sim-30 \mathrm{~nm}$ as the hole width is decreased from 286 to $148 \mathrm{~nm}$. This can be interpreted within the coupled-mode analysis as due to the fact that, when the hole size is decreased, the spectral location of the SPP in the corrugated surface tends to its corresponding value for a flat metal surface, with no holes.

In the extreme case of very large holes in a 2D array, the system becomes a metal wire mesh which, as stated in the Introduction, has been widely analyzed within the engineering community. These structures have been studied in several frequency regimes such as microwave (Keilmann, 1981), far infrared (Mitsuishi et al., 1963), midinfrared (Renk and Gensel, 1962; Ulrich, 1967), and infrared (Rhoads et al., 1982). They were either treated as transmission line equivalent circuits (e.g., inductive grids) or analyzed as diffraction gratings (Eggiman and Collin, 1962; Chen, 1971). Transmission properties of the mesh filters were eventually analyzed by considering the surface waves at the metal interface (Ulrich, 1974) in line with metal grating theory (Hessel and Oliner, 1965). Recently the analysis of the crossover from the EOT regime (small holes) to the wire mesh limit has been

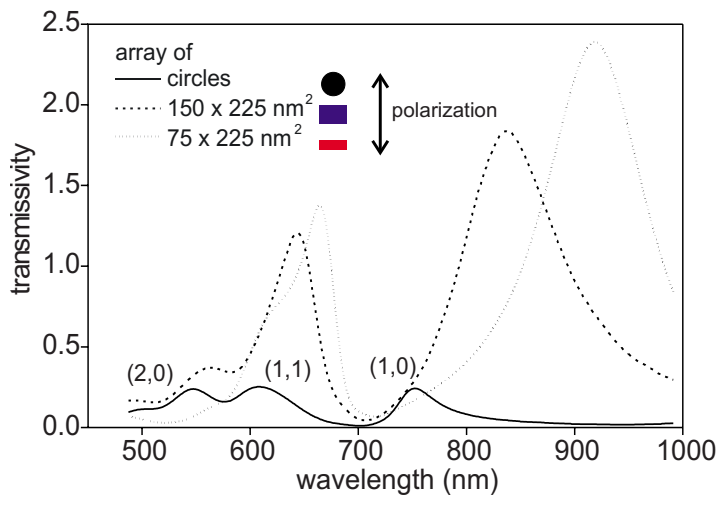

FIG. 35. (Color online) Measured transmission spectra for three different hole shapes: circular (diameter $190 \mathrm{~nm}$ ), rectangular $\left(150 \times 225 \mathrm{~nm}^{2}\right.$; aspect ratio 1.5$)$, and rectangular $(75$ $\times 225 \mathrm{~nm}^{2}$; aspect ratio 3 ). As the aspect ratio of the holes is increased from 1 to 3 , the normalized transmission of the primary peak increases by one order of magnitude. From Koerkamp et al., 2004.

addressed both experimentally and theoretically (BravoAbad, Martin-Moreno, et al., 2007).

The role of the individual holes in the EOT phenomenon in 2D hole arrays becomes important when the shape of the holes is changed from circular or square to rectangular (Koerkamp et al., 2004). Several key changes occur. First, the normalized transmission is increased by nearly one order of magnitude even though the actual area per hole decreases (see Fig. 35). This is in agreement with the trend observed in individual rectangular apertures, as described in Sec. II.B.2. Also, a strong polarization anisotropy occurs between the best transmitted polarization, i.e., perpendicular to the long axis of the holes, and the polarization along the long axis of the holes. This anisotropy, which causes birefringence is also observed for elliptical hole shapes (Elliott et al., 2004; Gordon et al., 2004) and has been addressed theoretically (Strelniker, 2007), confirming the experimental results. The birefringence exhibits a pronounced wavelength dependence (Elliott et al., 2005), thus opening up a large perspective for polarization control through tuning the aspect ratio of the holes, the wavelength, and the angle of incidence (Sarrazin and Vigneron, 2004). The same polarization effects have been observed for the transmission of terahertz radiation through arrays of rectangular subwavelength holes even though the thickness of the metal layer, in that case, is only a small fraction of the incident wavelength (Cao and Nahata, 2004a).

But perhaps the most profound change to the transmission spectrum shown in Fig. 35 is the observation that the primary $(1,0)$ peak undergoes a huge redshift when hole shape is varied (Koerkamp et al., 2004). A shift as large as $170 \mathrm{~nm}$ can be observed. In these first experiments the hole area was not kept constant and, while unlikely, this may have influenced the experiments.

In an investigation where only the aspect ratio of rectangular holes was varied, a smooth increase of the nor- 

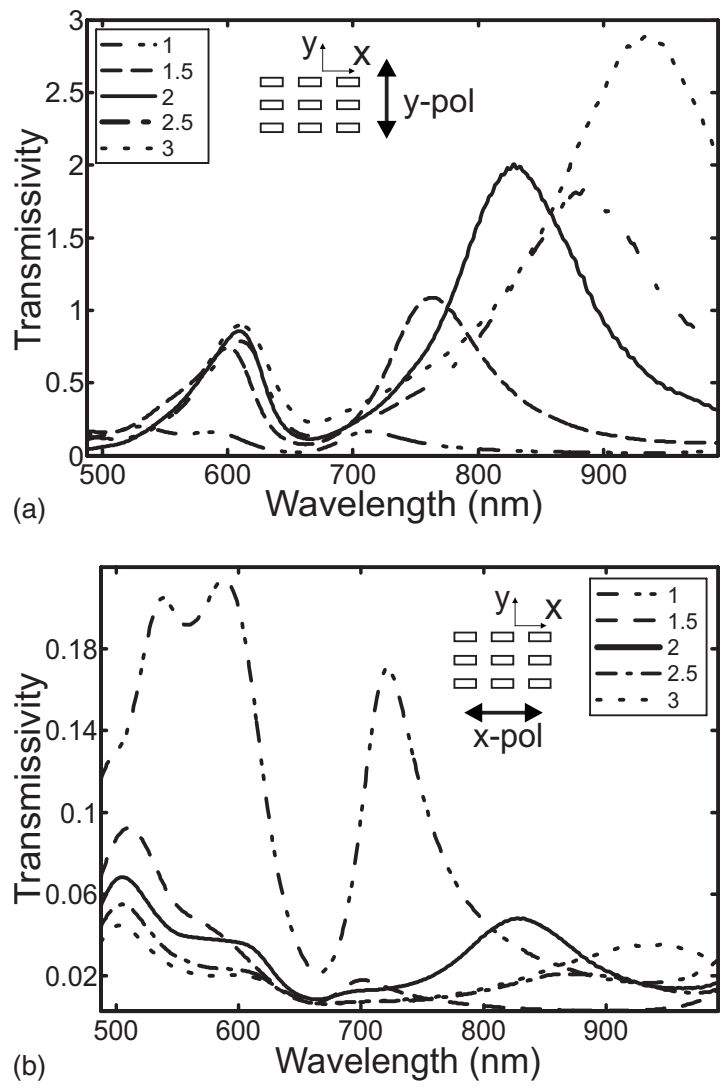

FIG. 36. Measured normalized transmission of nanohole arrays consisting of rectangular holes with varying aspect ratio for two different incident polarizations. The legends show the aspect ratios of the holes. The insets show the relative direction of the polarization with respect to the hole geometry. (a) The normalized transmission exhibits an increase for all peaks. The $(1,0)$ peak exhibits broadening and a pronounced redshift as the aspect ratio is increased. (b) For $x$-polarized light the opposite trend is observed: an increase in aspect ratio results in a decrease in normalized transmission. For the largest aspect ratios a small amount of polarization cross-talk is observed that can be attributed to minute alignment imperfections. From van der Molen et al., 2005.

malized transmission is observed in addition to a monotonic redshift as a function of the aspect ratio (van der Molen et al., 2005); see Fig. 36. For hole arrays with elongated holes the enhanced transmission is mediated by a combination of localized effects associated with the transmission properties of single rectangular holes and collective effects related to the periodicity of the array. The spectral position of the main $(1,0)$ peak shifts monotonically as the aspect ratio of the holes is increased from aspect ratios where periodicity governs the peak position, i.e., the aspect ratio equal to 1 , to aspect ratios where localized effects are important. Figure 36 also shows that the polarization anisotropy increases when the aspect ratio of the holes becomes larger. The large polarization anisotropy combined with the peak shifts induced by high aspect ratios can be exploited for practical purposes (see Sec. V.A).

The dependence of the transmittance through periodic hole arrays on the period, for a fixed hole shape,
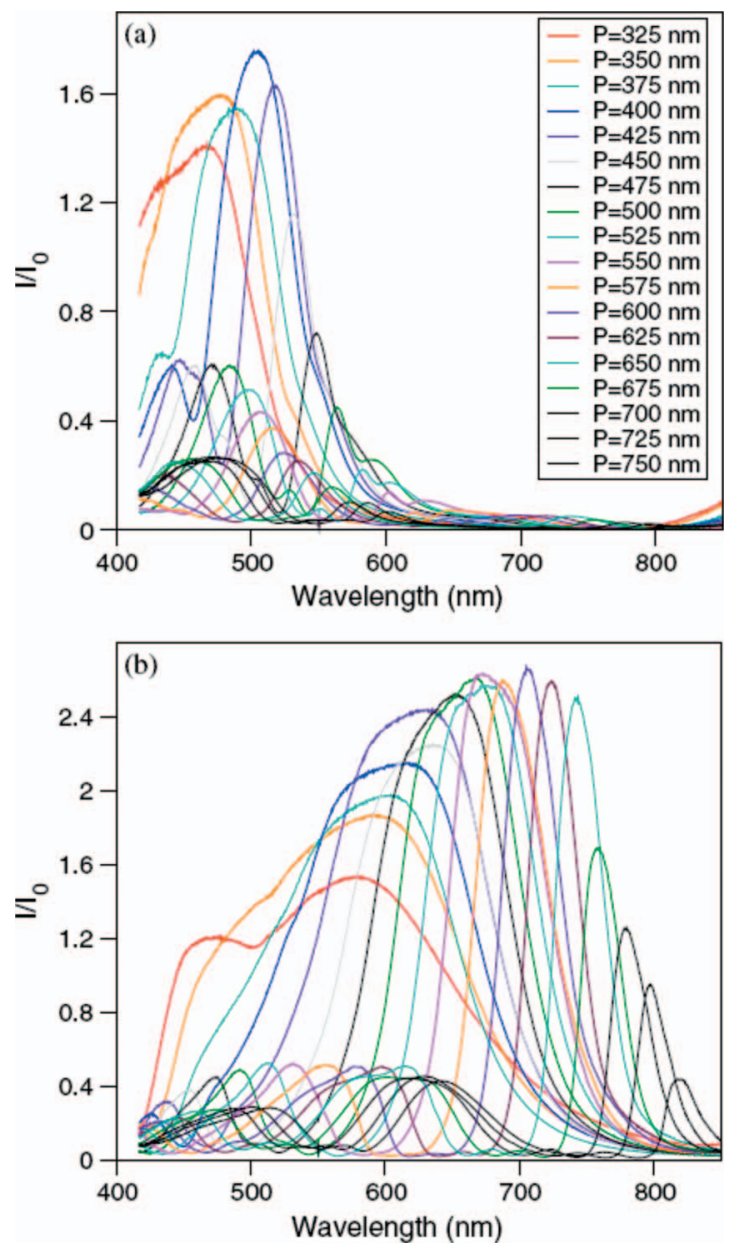

FIG. 37. (Color) Transmission spectra of rectangular holes with the lattice constant as a varying parameter, plotted for the polarization (a) perpendicular to the short edge and (b) perpendicular to the long edge. All samples were fabricated with the same rectangular holes $\left(260 \times 200 \mathrm{~nm}^{2}\right)$ perforated on suspended Ag films of thickness $400 \mathrm{~nm}$. From Degiron and Ebbesen, 2005.

was experimentally studied by Degiron and Ebbesen (2005). The measured transmittance spectra through a suspended Ag film pierced with rectangular holes are shown in Fig. 37 for the two orthogonal polarizations. The different contributions to the transmittance are clearly visible as the period changes. The SPP mode associated with the periodicity dominates the transmittance and the contribution of the localized mode is either blocked or revealed, depending on whether it coincides with a minimum or maximum of the SPP mode, respectively. At the same time, SPP transmission peaks attenuate above $550 \mathrm{~nm}$ when the polarization is perpendicular to the short axis of the aperture [see Fig. 37(a)], as compared to being perpendicular to the long axis [Fig. 37(b)]; this is due to a drastic shift in the cutoff wavelength of the individual apertures when going from the long to the short axis of the holes. This picture of the evolution and mixing between the transmission resonances associated with SPP and localized modes has been corroborated theoretically (Mary et al., 2007). 


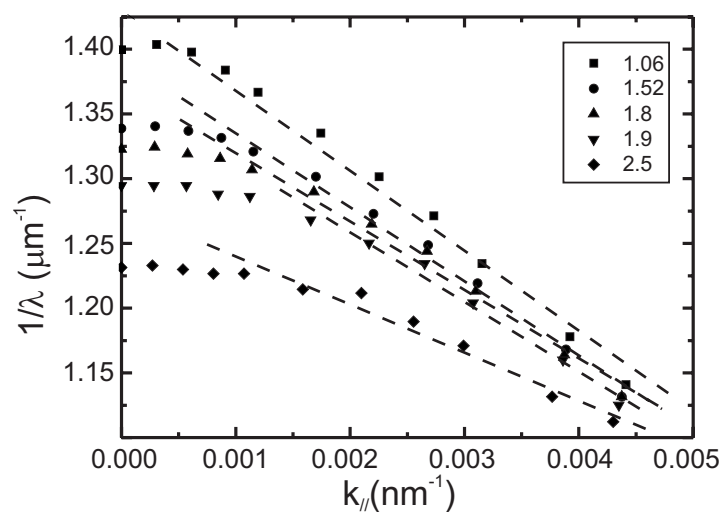

FIG. 38. Measured dispersion of the $(1,0)$ peak for different aspect ratios. The dispersion changes as the aspect ratio is increased: the fitting lines decrease in slope and the dispersion around $k_{\|}=0$ becomes flatter for a larger range of $k_{\|}$. This indicates a stronger degree of localization in the transmission process. From van der Molen et al., 2005.

Measurements show that hole shape also affects the dispersion of the EOT peaks. In particular, the flat region in the dispersion around $k_{\|}=0$ widens as the aspect ratio is increased (Fig. 38). This indicates that the mechanism responsible for the transmission becomes more localized in real space, which has been confirmed with near-field optical microscopy for rectangular holes with high aspect ratios (Chu et al., 2007).

The role of local effects can be enhanced through other hole shapes. Inspired by the fact that a coaxial cable has no cutoff, a periodic arrangement of annular or coaxial holes was proposed as an ideal candidate to show EOT in small apertures (Baida and Van Labeke, 2002, 2003). However, it was soon realized (Baida et al., 2004) that this propagating mode cannot be excited by a normally incident plane wave and it even plays a minor role for oblique incidence and $p$-polarized light (Baida, 2007). Instead, the transmission resonances emerging in arrays of annular holes result from the interplay between SPP modes and localized resonances that are spectrally located at the cutoff wavelength of the $\mathrm{TE}_{11}$ mode of the annular waveguide (Orbons and Roberts, 2006; Lomakin et al., 2007). Through an optimization of parameters, absolute transmissions were predicted up to $90 \%$ for arrays with a significant "opaque" area. The behavior of the annular holes has been alternatively described in terms of coupled cylindrical SPPs on the inner and outer surfaces of the annulus (Haftel et al., 2006). It was also predicted that square coaxial structures would exhibit the same behavior and allow large transmission coefficients (Moreau et al., 2003).

The theoretical predictions for the annular hole arrays were experimentally verified in the near infrared. Careful polarization and angle-dependent measurements confirm an interplay between cutoff resonances in the coaxial holes and the delocalized resonances associated with SPPs (Fan et al., 2005). It was shown that in the midinfrared the arrays of coaxial holes could transmit up to five times more than arrays of circular holes with the same open air fraction (Fan et al., 2005a, 2005b). In the visible range $(700 \mathrm{~nm})$, a maximum transmission of $17 \%$ was achieved (Salvi et al., 2005; Poujet et al., 2006). The normalized transmission through annular hole arrays can be further enhanced by choosing the proper symmetry of the array (Sun, Tian, Han, et al., 2006). Under certain circumstances and using the proper cavity resonances in the annular holes, the transmission of annular hole arrays can even become independent of the arrangement of the holes with respect to each other (Rockstuhl et al., 2007). The role of cylindrical SPPs was experimentally confirmed through experiments in combination with simulations and a consideration of the dispersion of these SPP modes (Orbons et al., 2007).

Periodic subwavelength hole arrays with more exotic hole shapes have also been shown to exhibit EOT, such as arrays of diamond-shaped holes (Sun, Liu, et al., 2006) and triangles (Kim and Moyer, 2006a, 2006b). A range of hole shapes has successfully been explored in order to redshift the cutoff wavelength of the individual holes. In particular, H-shaped holes (Sun et al., 2007) and cruciform-shaped holes (Chen, Tsai, et al., 2007; Ye, Wang, Cao, et al., 2007; Ye, Wang, Yan, et al., 2007) seem especially useful for this purpose. The use of shape in combination with array symmetry and periodicity provides a highly versatile tool box to fabricate, for example, terahertz filters (Lee, Seo, et al., 2006). Arrays consisting of double holes that slightly overlap provide an additional localized field enhancement near the resulting cusps (Gordon et al., 2005; Kumar and Gordon, 2006), which can be exploited for enhancing nonlinear phenomena (see below). Recently, evidence has been found that the structure in the holes perpendicular to the film can also influence the transmission. Measurements in the terahertz regime show that arrays with holes in which the radius first decreases and then increases to its original value exhibit blueshifts when the radius of the constriction in the holes is reduced (Batulla et al., 2007).

In summary, the EOT phenomenon in hole arrays is mediated by both localized and collective effects related to the periodicity of the array. The final spectral shape is dependent on the relative contribution of the cutoff function of the individual apertures, the localized modes, and the SPP modes and these are all sensitive not only to the lateral dimensions of the aperture but also to factors such as the depth of the apertures and the density of apertures in the metal film.

\section{Electric field enhancement and nonlinear effects}

Calculations showed that EOT is accompanied by highly localized field distributions (Krishnan et al., 2001; Salomon et al., 2001). Near-field experiments were able to confirm these predictions (Hohng et al., 2002) by taking advantage of the subwavelength nature of the EM fields associated with SPP excitation. Interestingly, high field amplitudes between the holes accompany certain maxima in the far-field transmission. In the near field, different components of the diffraction interfere to set 
up complex field patterns (Hohng et al., 2005). The exact spatial field distributions are determined by the spatial potential experienced by the Bloch modes (Zayats et al., 2003). The free-space wavelength with respect to the periodicity is also an important factor in the field patterns. When the free-space wavelength is much smaller than the periodicity of the array, clear standing-wave patterns between the holes can be observed (Rokitski et al., 2005b; Gao et al., 2006; Hou et al., 2006). In that case, an amplitude modulation persists away from the interface, whereas for larger wavelengths, as only the zero-order diffracted beam is radiative, a homogeneous pattern is found (Hohng et al., 2005). Even for quasiperiodic hole arrays hot spots with a diameter of roughly half the wavelength can be found up to 18 wavelengths away from the structure (Huang, Zheludev, et al., 2007).

On the other hand, these high EM fields that accompany the EOT phenomenon can be exploited to enhance nonlinear effects. Second harmonic generation (SHG) can arguably be considered as the most basic nonlinear optical effect. The first observation of enhanced SHG in a subwavelength hole was actually not in a hole array but in a bull's eye structure (see Sec. IV) in which the subwavelength hole was surrounded by a set of concentric grooves (Nahata et al., 2003). An enhancement of $10^{4}$ was found with respect to the second harmonic yield obtained from a single hole without grooves. The first observation of SHG in a hole array found a maximum yield for those incidence angles where the fundamental beam is maximally transmitted (Airola et al., 2005). A fivefold enhancement of the second harmonic yield with respect to the yield of disordered arrays was found. By breaking the centrosymmetry of the individual holes the yield was further increased.

The second harmonic yield can also be increased by changing the hole shape while retaining the centrosymmetric nature of both the holes and the array, for example, using rectangular holes. In the first instance this is not surprising: as the aspect ratio is varied, the linear transmission through the arrays is influenced. The nonlinear response may be expected to mimic the change in the linear response, albeit in a nonlinear fashion depending on the order of the nonlinear process being considered. Figure 39 shows both the linear transmission of the fundamental beam and the second harmonic yield behind the array as a function of the aspect ratio of the holes. For aspect ratios ranging from 0.36 to 1.6 an increase in the linear transmission by a factor of 60 is observed. At the same time the second harmonic yield is found to increase by a factor of 4000 .

However, a closer look at Fig. 39 shows that for aspect ratios ranging from 1.6 to 2.8 the transmission of the fundamental beam does not exhibit any increase. Nevertheless, the second harmonic yield is found to exhibit an additional increase by a factor of 50 before showing a decrease. In order to put this observation in perspective, it is useful to derive the effective nonlinear susceptibility of the structure. By considering the array as an effective homogeneous medium, the nonlinear susceptibility can be calculated from the second harmonic yield and all

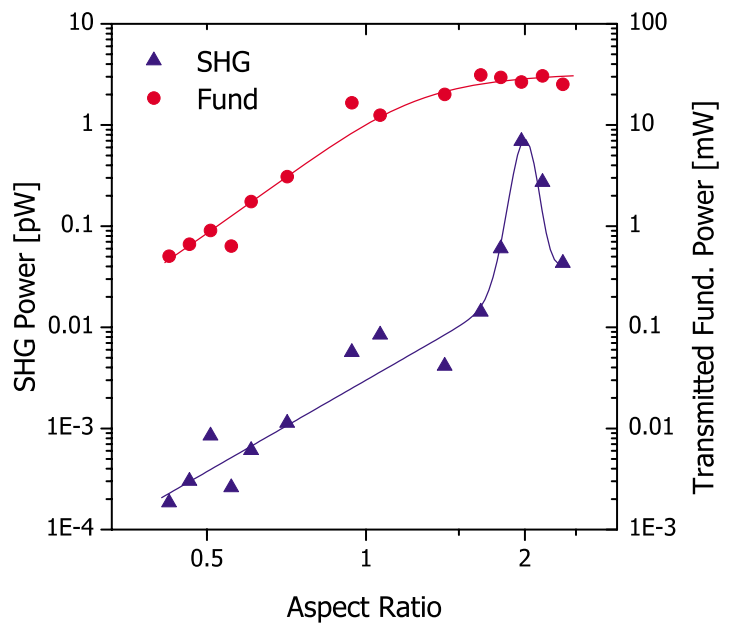

FIG. 39. (Color online) Double logarithmic plot of transmission of the fundamental beam at $830 \mathrm{~nm}$ (right axis, dots) and the collected second harmonic signal (left axis, triangles) as a function of aspect ratio. The transmission of the fundamental beam exhibits a monotonic increase before leveling off at an aspect ratio of 1.6. The second harmonic shows a steady increase of slightly less than two orders of magnitude up to an aspect ratio of 1.6. Between aspect ratios of 1.6 and 2.3 a peak is observed in the second harmonic with a magnitude of a factor 50. The lines are guides for the eye. From van Nieuwstadt et al., 2006.

relevant linear transmission coefficients (Sutherland, 1996). Figure 40 shows the nonlinear susceptibility of the hole arrays as a function of the aspect ratio of the holes. For aspect ratios ranging from 0.36 to 1.6 it can be seen that the nonlinear susceptibility exhibits no clear trend

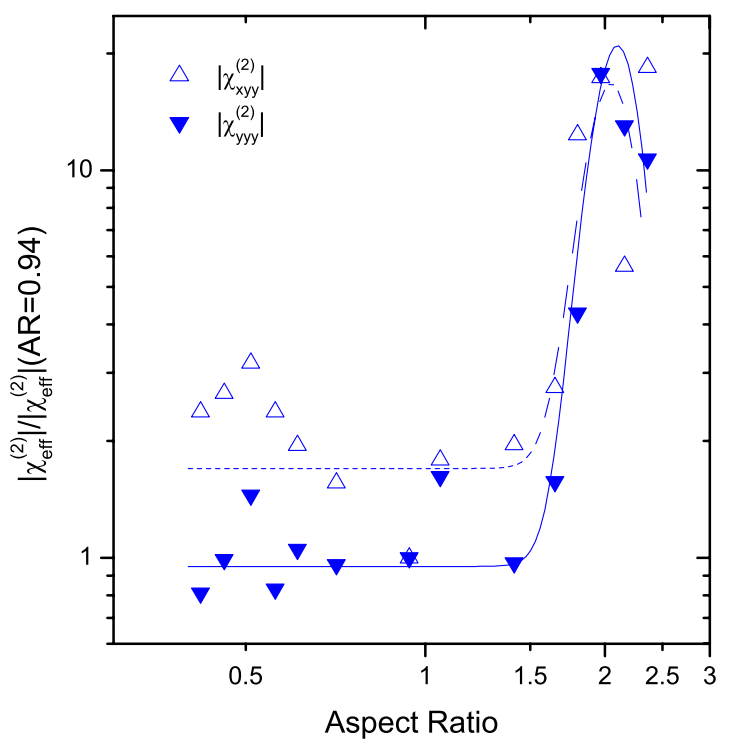

FIG. 40. (Color online) Double logarithmic plot of two components of the effective second-order nonlinear susceptibility vs aspect ratio. Between aspect ratios of 0.36 and 2.8 no clear trend is observable in the effective nonlinear susceptibility. Between aspect ratios of 1.6 and 2.8 a peak in the effective susceptibility of a factor more than one order of magnitude occurs. From van Nieuwstadt et al., 2006. 
as a function of aspect ratio. For aspect ratios of 1.6-2.8 a clear peak in the nonlinear susceptibility is found. An increase of the susceptibility of a factor of roughly 10 is observed (van Nieuwstadt et al., 2006). In other words, a "hot" hole shape is observed for aspect ratios around 2. This hot hole shape can be explained by the cutoff resonance condition of the individual rectangular holes. Associated with the excitation of this cutoff resonance, a huge EM field enhancement occurs, as explained in Sec. II.B.2, resulting in an enhanced nonlinear response.

The field enhancement in coaxial hole arrays has been exploited to generate a second harmonic with very high efficiency. It has been demonstrated that second harmonic generation from GaAs, which is introduced in the gaps of a coaxial hole array, can be as efficient as SHG from z-cut $\mathrm{LiNbO}_{3}$ (Fan, Zhan, Panoiu, et al., 2006). It turns out that the field enhancement inside circular hole arrays is also sufficient to generate a good second harmonic yield when GaAs is introduced in the holes (Fan, Zhang, Malloy, et al., 2006). The SHG in circular hole arrays, without GaAs, can be enhanced through the use of double hole geometries in which two overlapping holes lead to a sharp ridge between the holes that induces a high local enhancement. The SHG efficiency can be boosted by a factor of 14 with respect to circular holes (Lesuffleur, Kumar, and Gordon, 2007). The SHG process can be improved by tuning a linear transmission resonance to the second harmonic wavelength through a proper change in array periodicity or incidence angle for a wide range of hole and array symmetries $(\mathrm{Xu}$ et al., 2007).

So far only a limited number of investigations have used hole arrays to boost third-order nonlinear processes rather than the second-order process of SHG. By exploiting Kerr nonlinearity it is possible to time-gate one beam of photons being transmitted through a circular hole array with a second beam (Smolyaninov et al., 2002). The change in polarization of the beam that was switched shows that the enhanced fields inside the holes are crucial for an efficient switching. Kerr nonlinearity can lead to bistability in the extraordinary transmission as shown theoretically by Porto et al. (2004) and experimentally by Wurtz et al. (2006). In this case the high intensity of a control laser induces changes of the local index of refraction. This leads to a modification in the transmission which persists when the intensity is subsequently reduced, resulting in a characteristic hysteresis loop in the transmission as a function of the intensity of the control field. Two-dimensional FDTD calculations of subwavelength gratings have identified the layer thickness of the Kerr medium and the size of the apertures as crucial parameters for maximizing the bistability (Min et al., 2007).

\section{Finite-size effects}

The question of the dependence of EOT efficiency on the number of apertures has also been addressed from both the experimental and theoretical points of view. As EOT results from the collective response of the array,
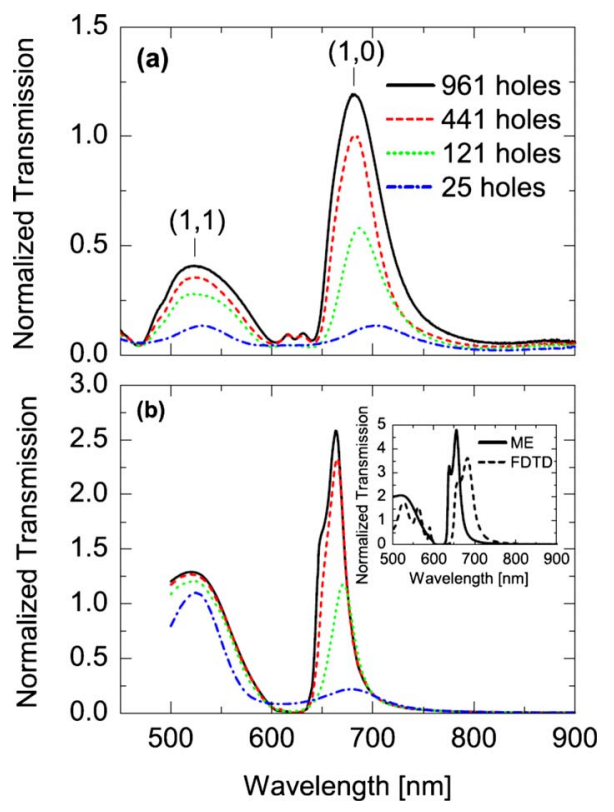

FIG. 41. (Color online) Dependence of EOT with the number of holes. (a) Experimental transmission spectra for 2D square arrays containing $5 \times 5,11 \times 11,21 \times 21$, and $31 \times 31$ circular holes. The arrays were milled in 275 -nm-thick free-standing silver films with a period $d=600 \mathrm{~nm}$ and a hole radius $r=134 \mathrm{~nm}$. Transmissions are normalized to the hole area. (b) Normalized-to-area transmission spectra obtained from the coupled mode method in which SIBC have been applied. The geometrical parameters are the same as in the experiments. Inset: Comparison between the coupled mode and FDTD results for an infinite square array. From Przybilla et al., 2008.

the resonant transmittance should increase as the number of holes forming the array increases until it reaches saturation. Indeed, experimental measurements in the infrared (Thio et al., 1999), in the terahertz regime (Miyamaru and Hangyo, 2004), and in the visible range of the EM spectrum (Henzie et al., 2007) have confirmed this expectation. Recently Przybilla et al. (2008) studied how the transmission per hole reaches saturation with the number of holes (see Fig. 41). They found, both experimentally and theoretically, that the number of holes needed for saturation depends very strongly on the size of the holes: the larger the hole diameter, the faster a transmission saturation is reached (see Fig. 41). This saturation value is marked by the propagation length of the SPP in the corrugated surface, which is mainly controlled by the size of the hole.

Przybilla et al. (2008) also analyzed the value of the EOT transmittance in large arrays and compared it to the transmittance through a single hole. The experimental results showed that the enhancement in the transmittance per hole in arrays is larger for the smaller holes: this enhancement is around 40 for holes with a diameter of $150 \mathrm{~nm}$ in $40 \times 40$ arrays (see Fig. 42). The computed enhancement for these parameters was around 100 and much larger values are obtained for smaller holes and larger array sizes. In contrast to some claims that the maximum enhancement achievable in EOT with respect 

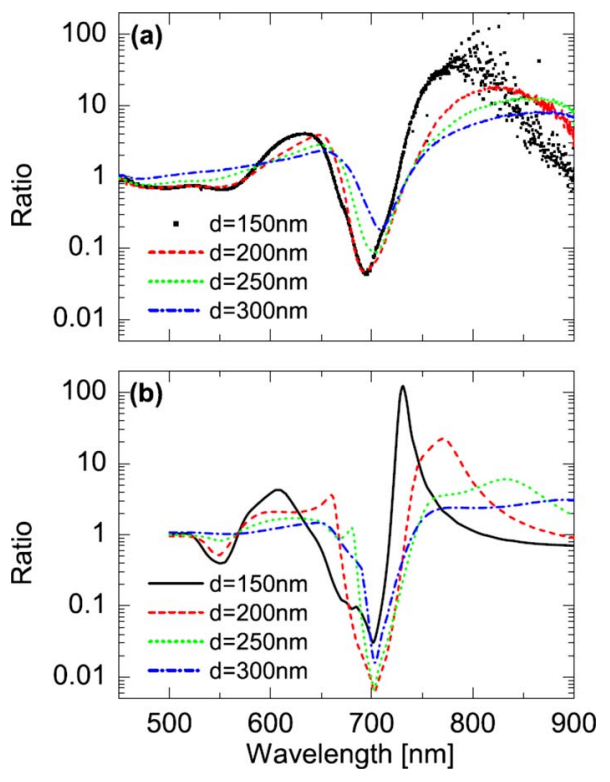

FIG. 42. (Color online) Dependence of the transmission enhancement with the diameter of the holes. (a) Experimental and (b) theoretical ratios of the transmission of $40 \times 40$ arrays to the transmission of the corresponding single hole with diameters of 150, 200, 250, and $300 \mathrm{~nm}$. All data are presented in a logarithmic scale for better visualization. From Przybilla et al., 2008.

to the single hole transmittance is around 7 (Lezec and Thio, 2004), no fundamental upper limit exists to the possible enhancement attainable in the EOT phenomenon, provided the system can be described within the macroscopic Maxwell equations.

Finite-size effects have also been theoretically analyzed in 1D arrays of holes and slits. For the case of holes, it was found that a chain of subwavelength holes can be considered as the basic geometrical unit showing EOT. A 2D hole array can be seen as a collection of weakly coupled $1 \mathrm{D}$ chains of holes oriented along the in-plane component of the $E$ field (Bravo-Abad, GarciaVidal, and Martin-Moreno, 2004). Regarding slits, the main parameter controlling the saturation of the resonant transmission associated with surface EM modes is the ratio between the width of the slits and period of the array (Fernandez-Dominguez et al., 2007). As expected, the other channel for transmission, linked to the excitation of a slit-cavity resonance, does not depend strongly on the number of slits in the array.

The spatial dependence of the transmitted radiation also presents strong finite size effects. Both experiments and theory (Bravo-Abad et al., 2006) showed that the reemission pattern is far from uniform (see Fig. 45). For instance, for a hole array of square shape [see Fig. 45(b)], the transmission per hole is maximum at the center of the sample, decreasing to the edges of the array. Additionally, this reemission pattern is sensitive to the angle of incidence. By tilting the samples by just a few degrees, the central band of maximum transmittance in Fig. 45(b) moves to the edge of the array. The strong nonuniformity of the light emerging from the hole array is also reflected in time-resolved measurements in the near-field region (Kim et al., 2003; Muller et al., 2003); see Sec. III.B.8. Interestingly, light emerging from small arrays also presents very low divergence. It has been shown experimentally that $75 \%$ of the transmission is actually nondivergent in arrays formed by just 16 holes, opening perspectives for microscopy applications (Docter et al., 2006; Chowdhury et al., 2007).

\section{Polarization effects}

Polarization effects associated with the shape of the holes have already been discussed in Sec. II.B.2 for single holes and in Sec. III.B.4 for 2D arrays. Here we focus on polarization effects due to the symmetry of the periodic array in relation with the polarization of the incident light.

The coupling of the incident plane wave with the surface EM modes is much more efficient when the direction of the incoming $E$ field, as projected on the interface, has a nonzero component in the direction of a reciprocal-lattice vector. For example, for a simple rectangular lattice, this means that if the normally incident light is polarized along the $x$ direction, potential resonances owing to reciprocal lattice vectors pointing in the $y$ direction will not lead to strong transmission peaks. The influence of the polarization leads to a rich transmission behavior. Depending on the projection of the incident $E$ field on the various periodicities present in the lattice, different leaky surface modes may be excited, each of which will in turn lead to a transmission resonance with its own amplitude, polarization direction, and phase; together they determine the polarization state of the transmitted light. The simplest consequence is that, when the sample is tilted away from normal incidence, a geometrical birefringence is introduced. This was first confirmed for terahertz transmission (Miyamaru et al., 2003) and later for optical frequencies (Ren et al., 2007). The full richness of the polarization behavior was investigated for optical frequencies (Altewischer et al., 2003). For square lattices, normal incidence, and polarization along the $(1,1)$ direction, SPP modes can be excited in both the $x$ and $y$ directions with the same efficiency and phase to the extent that the paths become indistinguishable when farfield experiments are performed.

The ultimate proof of this indistinguishability was given when polarization-entangled photons were transmitted through the subwavelength hole array with only a minimal loss to the degree of entanglement (Altewischer et al., 2002; Guo, Ren, et al., 2007). By changing the focus of the experimental configuration, the interplay between polarization and the dispersive propagation of the SPP modes led to a deterioration of the degree of entanglement. It was subsequently found theoretically (Moreno, Garcia-Vidal, Erni, et al., 2004; Altewischer, Oei, et al., 2005; Genet et al., 2005) and confirmed experimentally (Altewischer, Genet, et al., 2005) that the beam transmitted by a hole array was depolarized through a combination of a nonlocal response of the 
array due to SPP propagation and to the spread of wave vectors in the incident beam. The classical depolarization of a transmitted polarized beam and the quantum decoherence experienced by a polarization-entangled photon pair can be compared (Altewischer, van Exter, and Woerdman, 2005). Under certain conditions the quantum visibility and the classical degree of polarization can be found to be the same. It was also shown that a coupling exists between the temporal and spatial decoherence channels, which may be attributed to transverse propagation of SPPs (Lee, van Exter, and Woerdman, 2006).

Another way to deal with the polarization of the transmitted beam is by working with $2 \mathrm{D}$ hole arrays made of elliptical holes arranged in two sublattices in which the ellipses are orthogonally oriented. The polarization-induced frequency shift of the primary peak can be exploited to gain insight into the energy redistribution in the array (Masson and Gallot, 2006).

\section{Dynamics of the EOT phenomenon}

In order to gain further understanding of the EOT phenomenon, its dynamics was also investigated. It was found early that the transmission through a subwavelength hole array through a film with typical thicknesses of several hundreds of nanometers occurred on a femtosecond time scale (Dogariu et al., 2001, 2002). A transit time of $7 \mathrm{fs}$ was found for a film with a thickness of $300 \mathrm{~nm}$. Using the thickness as a distance traveled, this finding indicates a group velocity of $c / 7$. The transit time can be related to a lifetime of the resonant modes. This lifetime, which directly determines the linewidth of the transmission peaks, is, on the one hand, governed by electron-phonon coupling, which leads to Ohmic losses (van Exter and Lagendijk, 1988; Groeneveld et al., 1990). On the other hand, the SPP modes experience radiative losses directly related to the EOT phenomenon itself. It has been shown through ultrafast investigations that the SPP modes had coherent propagation lengths of only a few microns, which is consistent with a (sub-)10-fs lifetime. The radiative lifetime is limited by SPP scattering with the periodic array itself, which leads to the homogeneous broadening of the transmission peaks (Kim et al., 2003). Further investigations have shown that the light transmitted through the subwavelength hole arrays exhibits clear oscillations on a femtosecond time scale (Muller et al., 2003). The observation underpins the coupling between both interfaces of the metal layer through photon tunneling. The diameter of the holes has been found to determine the period and damping of these oscillations. In other words, the size of the subwavelength holes governs both the coupling between the interfaces and the radiation damping and hence the linewidth of the transmission peaks. This is in agreement with the theoretical modeling presented in Sec. III.B.2 and it has been corroborated by more sophisticated FDTD calculations (Müller et al., 2004). Similar ringing has also been observed for the transmission of terahertz pulses through subwavelength metal hole arrays on high resistivity silicon, albeit at different time scales owing to the large difference in frequency (Qu and Grischkowsky, 2004). The radiation-limited lifetime of the SPP modes is crucially influenced by the symmetry of the mode: for the so-called antisymmetric mode, the radiative damping time constant is reduced by almost one order of magnitude (Ropers et al., 2005, 2006). Visualization of the bound modes in time with sophisticated far-field experiments reveals their group velocity (Rokitski et al., 2005a).

The dynamic measurement of the transmission process (Kim et al., 2003; Muller et al., 2003), which exhibits a two-component structure of a fast transmission of a virtually unperturbed pulse followed by a single long tail, is the temporal fingerprint of a Fano-type process, as discussed in Sec. III.B.1. Careful investigation of the point spread function provides more proof for the transmission scenario through a Fano process (Altewischer, Ma, et al., 2005; Altewischer et al., 2006). Polarizationdependent distortions in near-field investigations confirm the conclusions obtained in far-field investigations (Mrejen et al., 2007).

\section{Quasiperiodic arrays}

\section{General results}

The first experimental study showing the emergence of EOT phenomenon in quasiperiodic arrangements of subwavelength holes was reported by Sun, Tian, Li, et al. (2006). They fabricated an eightfold quasiperiodic hole array with a parallelogram-square tiling system, with the side length of the parallelogram and square being about $550 \mathrm{~nm}$. A very broad transmission peak at $\lambda=700 \mathrm{~nm}$ dominated the transmission spectrum. They also pointed out that the long-range order existing in the quasiperiodic configuration makes it similar to a grating and provides some dominant discrete reciprocal vectors to assist the coupling of the incident EM field with the SPPs of the metal. The next experimental study showing this EOT phenomenon in the optical regime was by Przybilla, Genet, et al. (2006). They built up 2D Penrose tiles composed of two types of rhombuses that are matched to pave all the 2D planes with a fivefold symmetry. Figure 43 shows a typical experimental spectrum measured through a Penrose tile. Although periodicity is absent, well-defined peaks of enhanced transmission clearly emerge in the spectrum. They also correlated the spectral locations of the transmission maxima with peaks in the structure factors of the quasiperiodic arrangements (i.e., diffraction pattern of the array). Additionally, they analyzed the variation of the transmission peak heights with the number of holes $(N)$ showing that, as in the periodic case, there is a saturation as $N$ increases (associated with the finite mean free path of the SPPs excited by the incoming plane wave).

Matsui et al. (2007) presented an experimental analysis of the EOT phenomenon in aperiodic arrays of subwavelength holes in the terahertz regime. They also analyzed Penrose tiles exhibiting local fivefold rotational 


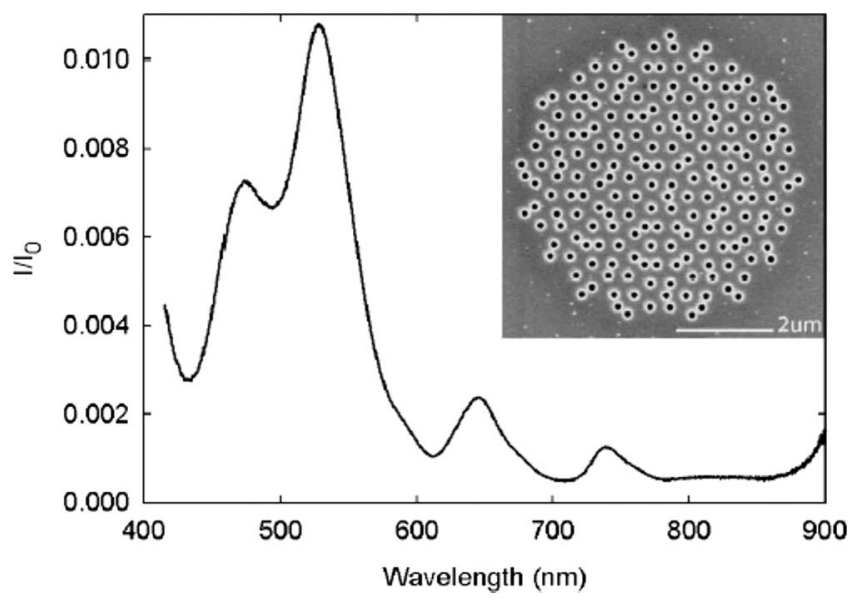

FIG. 43. Optical transmission spectrum of a Penrose array with a rhombus edge of $P=450 \mathrm{~nm}$ and a hole diameter of $150 \mathrm{~nm}$. The transmitted intensity $I$ is normalized to the incident one $I_{0}$. The inset is a SEM image of the array milled through a 300-nm-thick silver film. From Przybilla, Genet, et al., 2006.

symmetry, but additionally they considered Penrose lattices with tenfold symmetry, corroborating in both cases the close link between transmission peaks and resonant features of the structure factor. Furthermore, they designed an aperiodic array presenting 18-fold rotational symmetry in real space that was constructed by Fourier transforming a circular 2D diffraction pattern. In this aperiodic arrangement, EOT peaks also appeared and their magnitude was even larger than in the quasiperiodic case. Finally, Papasimakis et al. (2007) demonstrated both theoretically and experimentally that EOT in quasiperiodic arrays also emerges in the microwave range of the EM spectrum.

\section{Coupled-mode analysis}

The emergence of EOT phenomenon in quasiperiodic arrangements made it necessary to reconsider the theoretical explanation of EOT based on the excitation of surface EM modes, as described in Sec. III.B.2. In what follows we present a summary of the theoretical analysis based on the coupled mode method.

In order to present a complete picture, in this section we compare the transmission spectra of a periodic square lattice, a Penrose lattice, and a random distribution of circular holes; in all cases we consider $N=636$ holes. The ordered structure is a circular portion of a square lattice, with the lattice parameter chosen so that the external radius of the circular array is the same as in the quasiperiodic case. In the disordered case, the holes are randomly placed within the same external radius (but without overlapping). The considered geometrical values are typical of EOT experiments in the optical regime (Przybilla, Genet, et al., 2006). Note that, as we are assuming that the metal behaves as a PEC, our results are applicable to different frequency regimes, just by scaling all lengths by the same factor.

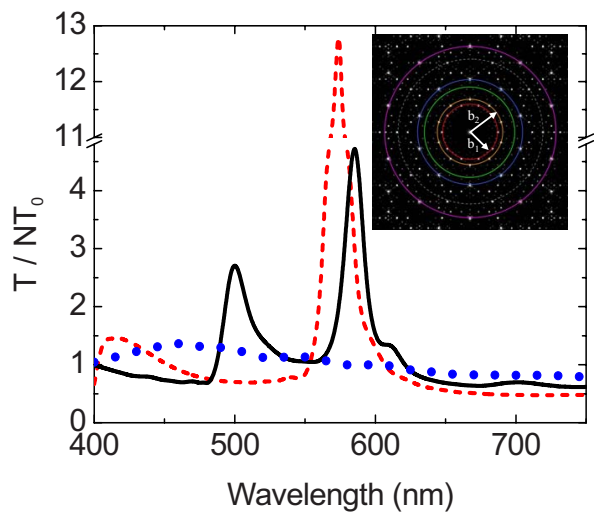

FIG. 44. (Color online) Transmission $(T)$ spectra for the ordered case (broken line), the Penrose lattice (continuous line), and the random configuration (dots). In all three cases, $r=130 \mathrm{~nm}, h=170 \mathrm{~nm}$, and $N=636$. $T$ is normalized to the value obtained for $N$ independent holes. The inset shows the structure factor of the Penrose lattice considered.

Figure 44 shows the corresponding optical transmission spectra evaluated at normal incidence. The transmittance for the collection of $N$ holes is normalized to $N$ times the normalized-to-area transmission through a single circular hole $T_{0}$, i.e., by the transmittance expected for a set of $N$ independent holes. In the ordered case (dashed line), the transmittance spectrum is smooth, with values close to those of independent holes $\left(T / N T_{0} \approx 1\right)$, except near the resonant peak appearing at $\lambda=575 \mathrm{~nm}$, where the transmission enhancement is around 13. This is the canonical EOT peak, appearing in periodic arrays at a resonant wavelength slightly larger than the lattice parameter $d=562 \mathrm{~nm}$. Resonant transmission also appears when holes are arranged in a Penrose lattice (solid curve in Fig. 44), in line with the experimental findings. In the numerical results, transmission enhancements of about 3 and 5 are obtained at the resonant wavelengths $\lambda=500$ and $585 \mathrm{~nm}$, respectively. In contrast, the transmission spectrum for the random array (dotted line in Fig. 44) does not show resonant feature, confirming the importance of longrange order in observations of EOT effects.

As explained the appearance of EOT in quasiperiodic distributions of holes has been phenomenologically related to the lattice structure in reciprocal space, extending arguments borrowed from the ordered case (Przybilla, Genet, et al., 2006; Sun, Tian, Li, et al., 2006; Matsui et al., 2007; Papasimakis et al., 2007). Next, we provide quantitative arguments which support this suggestion by working analytically in the reciprocal space, following the derivation presented by Bravo-Abad, Fernandez-Dominguez, et al. (2007), in which the structure factor of the array appears explicitly in the equations. The Fourier components of the modal amplitudes can be written as $E_{n}(\vec{q})=\sum_{\vec{R}} \exp (-i \vec{q} \cdot \vec{R}) E_{n}(\vec{R})$. Note that now $E_{n}(\vec{R})$ refers to the modal amplitude in real space of mode $n$ at the hole location $\vec{R}$. By applying a Fourier transform to the set of equations (1) [further details can 
be found in Bravo-Abad, Fernandez-Dominguez, et al. (2007)], the structure factor of a given distribution of holes $\left[S(\vec{q})=\Sigma_{\vec{R}} \exp (-i \vec{q} \cdot \vec{R})\right]$ appears explicitly in the set of equations governing $\left\{E_{n}(\vec{q}), E_{n}^{\prime}(\vec{q})\right\}$ [see Eq. (A24) of the Appendix].

The reillumination term in Eqs. (A24), $\Omega_{n}^{(\prime)}(\vec{q}) E_{n}^{(\prime)}(\vec{q})$, is now represented by the scattering process that couples $E_{n}^{(\prime)}(\vec{q})$ to the continuum $E_{m}^{(\prime)}(\vec{k})$, the momentum difference provided by the lattice through $S(\vec{q}-\vec{k})$ :

$$
\Omega_{n}^{(\prime)}(\vec{q}) E_{n}^{(\prime)}(\vec{q})=\sum_{m} \int d \vec{k} G_{m n ; \vec{k}} S(\vec{q}-\vec{k}) E_{m}^{(\prime)}(\vec{k}) .
$$

The amplitude of this process is governed by $G_{m n ; \vec{k}}$,

$$
G_{m n ; \vec{k}}=\frac{i}{(2 \pi)^{2}} \sum_{\sigma} Y_{\vec{k} \sigma}\langle n \mid \vec{k} \sigma\rangle\langle\vec{k} \sigma \mid m\rangle .
$$

As discussed, an important property is that $G_{m n ; \vec{k}}$ diverges whenever a $p$-polarized diffraction wave goes glancing $\left(k_{z}=0\right)$, as can be directly seen from Eq. (29). By examining Eq. (28), it is clear when and where resonant peaks would emerge in the transmission spectrum for quasiperiodic arrays. Whenever a divergence in $G_{m n ; \vec{k}}$ coincides with a peak in the structure factor $S(\vec{q})$, the reillumination from all other holes characterized by $\Omega_{n} E_{n}$ is maximum. In other words, for a PEC film, a hole is maximally reilluminated by the other holes in the array at wavelengths corresponding to the glancing condition for the main wave vectors of the structure factor. For the quasiperiodic array considered in Fig. 44, this happens for $\lambda_{1}=566 \mathrm{~nm}\left(\left|\vec{b}_{1}\right|=2 \pi / \lambda_{1}\right)$ and $\lambda_{2}=483 \mathrm{~nm}$ $\left(\left|\vec{b}_{2}\right|=2 \pi / \lambda_{2}\right)$ (see the inset of Fig. 44). Consequently, $T$ shows two minima at these two wavelengths. At slightly larger wavelengths, two transmission peaks appear in the spectrum, much in the same way as it occurs in periodic arrays. Note however, that, in the quasiperiodic case, there is no minimum wave vector for diffraction [i.e., the structure factor is nonzero for wave vectors with modula smaller than $\left|\vec{b}_{1}\right|$ (see the inset of Fig. 44)]. This results in diffraction onto additional radiative modes in vacuum (other than the zero-order mode), which leads to both smaller resonant peaks and less pronounced minima than those appearing in the periodic case.

The fact that surface EM modes are involved in EOT in quasiperiodic systems does not imply that the transmission distribution is uniform across the array. This fact is shown in Fig. 45, in which the transmission per hole in a Penrose lattice of $N=636$ holes at the two resonant wavelengths is plotted $[\lambda=500$ and $585 \mathrm{~nm}$ in Figs. 45(c) and 45(d), respectively]. For comparison, Figs. 45(a) and 45(b) show the corresponding distribution for the ordered array at the resonant wavelength of $575 \mathrm{~nm}$ for circular and square external shapes of the array, respectively. In all four cases, the incident $\mathbf{E}$ field is pointing along the $x$ direction. The pattern in the ordered case can be understood by consideration of the 2D hole array as a collection of weakly coupled 1D chains of holes
(Bravo-Abad, Garcia-Vidal, and Martin-Moreno, 2004). Due to finite-size effects, the transmittance is larger at the center of the chain than at the edges. In arrays with a square shape, with one of the axes of the square lattice oriented along the incident $E$ field, all "horizontal" chains have the same length, resulting in a characteristic pattern where the edge effects are more noticeable along the direction parallel to the incident field than to the perpendicular one (Bravo-Abad et al., 2006) [see Fig. 45(b)]. On the other hand, when the shape of the square array is circular, the number of holes within field-aligned chains reduces close to the top and bottom regions, and the transmission per hole presents its maximum values just at the central region of the system, as shown in Fig. 45(a).

In the quasiperiodic arrangement, the transmission per hole distribution presents a completely different pattern: it is far from being uniform, showing the appearance of some hot-spot holes with very high transmittance. Interestingly, for each resonant wavelength, hot spots show a similar local environment. For $\lambda=500 \mathrm{~nm}$, hot spots appear in the center of a pentagon defined by their nearest neighbors [see Fig. 45(c)] while for $\lambda$ $=585 \mathrm{~nm}$, hot spots are located within a stadiumlike structure [see Fig. 45(d)]. The emergence of these hot spots at the metal surface when light is transmitted through a quasiperiodic array of holes has been experimentally observed by taking advantage of their replication in the far-field through the Talbot effect (Dennis et al., 2007; Huang, Zheludev, et al., 2007).

\section{TRANSMISSION THROUGH SINGLE APERTURES FLANKED BY PERIODIC CORRUGATIONS}

\section{A. General results}

As discussed, the main ingredient for the appearance of EOT is leaky surface EM modes that couple to the incident light. This idea suggested that the EOT phenomenon should also occur in single apertures if they were surrounded by a finite periodic array of indentations. This hypothesis was experimentally verified (Thio et al., 2001; Lezec et al., 2002) both for a 1D slit surrounded by a finite array of grooves [see Fig. 46(a)] and for the bull's eye structure [a 2D cylindrical hole flanked by circular trenches; see Fig. 46(b)].

The dependence of the optical transmission in the 1D geometry with the period of the array and depth of the grooves was analyzed experimentally by Garcia-Vidal, Lezec, et al. (2003). The transmittance spectrum of a single slit presents a broad maximum, associated with a Fabry-Perot mode excitation, at around $\lambda=725 \mathrm{~nm}$ [see the black curve and the inset of Fig. 47(a)]. This figure also shows that, under the presence of a period array of grooves, an additional peak emerges in the spectrum and moves to longer wavelengths as $d$ is increased. The peak is strongest for $d=650 \mathrm{~nm}$ when it appears at a wavelength that coincides with the slit waveguide mode excitation, $\lambda=750 \mathrm{~nm}$. Figure $47(\mathrm{~b})$ shows that there is an optimum value for the depth of the grooves, which 

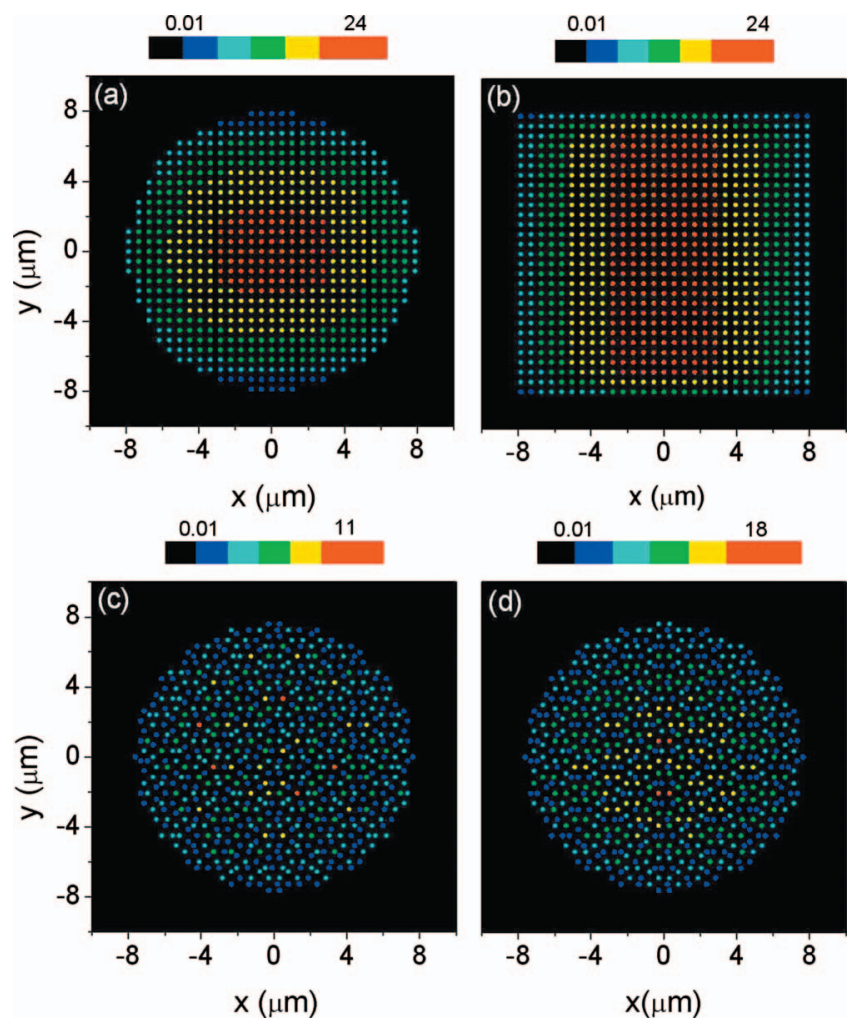

FIG. 45. (Color) Transmission per hole (normalized to the single-hole transmission) displayed in a color scale. (a), (b) Ordered arrays with circular and square external shapes, respectively. Both patterns were computed at the resonant wavelength of both structures: $\lambda=575 \mathrm{~nm}$. The results for the Penrose lattice at the corresponding resonant wavelengths are shown for (c) $\lambda=500 \mathrm{~nm}$ and (d) $\lambda=585 \mathrm{~nm}$. The geometrical parameters are the same as in Fig. 44.

for this particular set of geometrical parameters is around $40 \mathrm{~nm}$.

Experiments also showed that, unexpectedly, when the periodic corrugation was placed at the output surface of the aperture, the angular distribution of the transmitted radiation could be strongly modified (Lezec et al., 2002). At some resonant wavelengths, light emerged from the structure as a strongly collimated beam. Figure 48(a) shows the spectra of the angular distribution of transmitted radiation emerging from the exit side of a single slit surrounded by ten grooves milled in a free-standing silver film. As the collection angle separates from the normal direction, the transmission resonance splits into two smaller peaks. The evolution of the transmittance spectrum with the output collection angle presents a slightly different behavior for the $2 \mathrm{D}$ case, the bull's eye geometry, as shown in Fig. 48(b). Here a strong transmission peak is evident at $\lambda=660 \mathrm{~nm}$ with an intensity that strongly reduces as the collection angle increases. At the same wavelength, light emerges as a well-defined narrow beam with a full width at half maximum divergence of $\pm 5^{\circ}$. When the finite angular resolution of the experimental setup is taken into account, the actual beam divergence is estimated to be $\pm 3^{\circ}$. Atomic fluorescence mapping of the optical field inten-
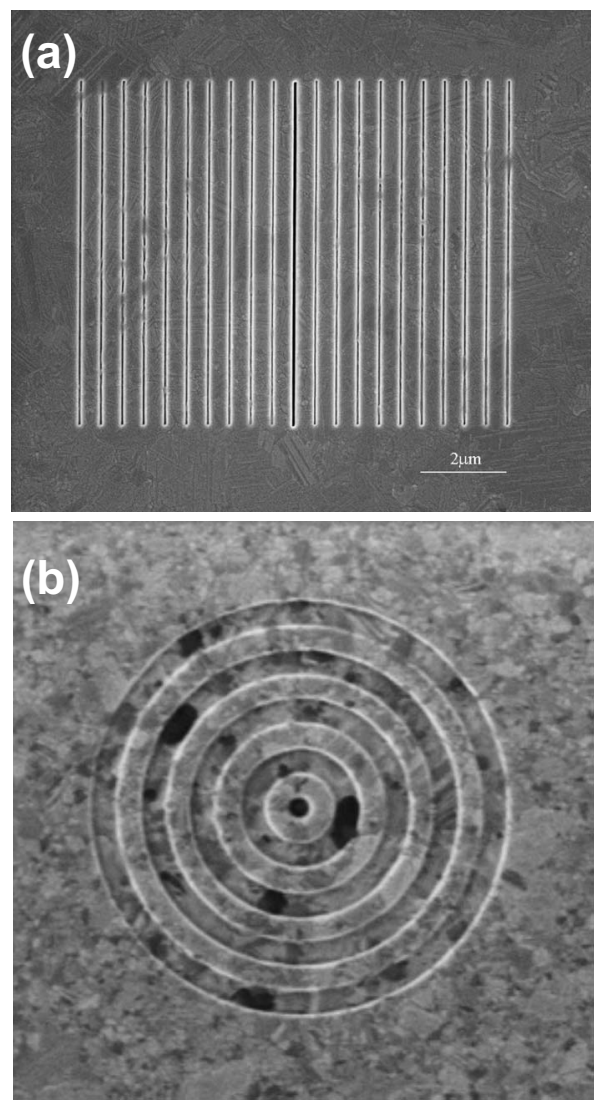

FIG. 46. SEM images of two samples showing a single aperture surrounded by periodic corrugations. (a) 1D case: a single slit symmetrically flanked by grooves. (b) $2 \mathrm{D}$ case: the socalled bull's eye structure, a cylindrical hole surrounded by circular trenches.

sity profile of these structures suggests an even smaller beam divergence (Gay et al., 2005).

Experiments have shown that the total transmission through a bull's eye structure is the product of three distinct contributions: the coupling efficiency on the input surface, the transmission or cutoff function of the aperture, and the decoupling efficiency of the output surface (Degiron and Ebbesen, 2004). It was found that the SPP grating modes of the input surface dominate the bull's eye spectrum, although the contribution of the localized mode of the central hole can still be detected as in the case of hole arrays (see Sec. III.B). The beaming effect is, however, controlled by the corrugations of the output surface as further discussed in Sec. IV.B.

As a technical note, the bull's eye geometry also forms the basis of choke ring antennas, extensively used in the microwave regime for global positioning system applications (Tranquilla et al., 1994). These antennas are used to suppress the signal at the center coming either from the horizon or from diffraction by the edge of the metal ground plane and not to enhance the signal at the center. For this, the corrugation in choke ring antennas is used to suppress the surface EM modes present in the flat uncorrugated surface. This is achieved using a corrugation period much smaller than the wavelength and a depth such that the corrugated metal surface spoofs a 


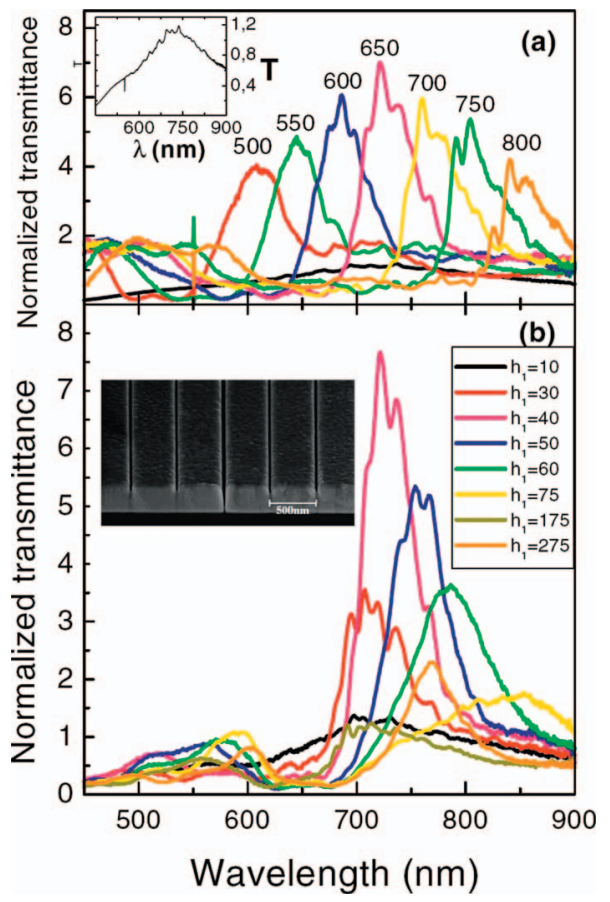

FIG. 47. (Color) Normalized-to-area transmittance spectra for different structures formed by a central slit symmetrically flanked by ten grooves on the input surface. The width of the slits is $40 \mathrm{~nm}$ and the thickness of the free-standing silver film is $h=350 \mathrm{~nm}$. (a) In these spectra the depth of the grooves is fixed at $w=40 \mathrm{~nm}$ and the period of the groove array $d$ is varied between 500 and $800 \mathrm{~nm}$. The black curve corresponds to the single-slit case that is also reproduced in the inset for clarity. (b) Here the period is fixed, $d=650 \mathrm{~nm}$, and the depth of the grooves is changed from $w=10 \mathrm{~nm}$ up to $275 \mathrm{~nm}$. The inset shows a SEM image of one of the devices analyzed. From Garcia-Vidal, Lezec, et al., 2003.

dielectric one. In contrast, the corrugation in the bull's eye geometry for EOT purposes is used to tailor the properties of surface EM modes, or even to create them, as shown below.

Both enhanced transmission and beaming phenomena in single apertures surrounded by periodic corrugations have been observed in other frequency regimes. Within the microwave range of the EM spectrum, Hibbins et al. (2002) presented a study of the enhanced transmission of radiation through a single slit placed at the center of a pair of grooves. The beaming effect in 1D structures at this frequency range was analyzed by Akarca-Biyikli et al. (2004), reporting a $4^{\circ}$ angular divergence for a system with ten grooves. The $2 \mathrm{D}$ case (bull's eye geometry) at microwave frequencies was first addressed by Lockyear et al. (2004, 2005). In this last reference, a single hole surrounded by four circular trenches also presented both enhanced transmission and beaming effects.

In the terahertz range of the EM spectrum, it was possible to analyze the phenomenon of enhanced transmission for the 2D bull's eye geometry in the time domain (Agrawal et al., 2005), allowing a detailed study of the transmission process. They were able to determine the contribution of each individual groove to the trans-
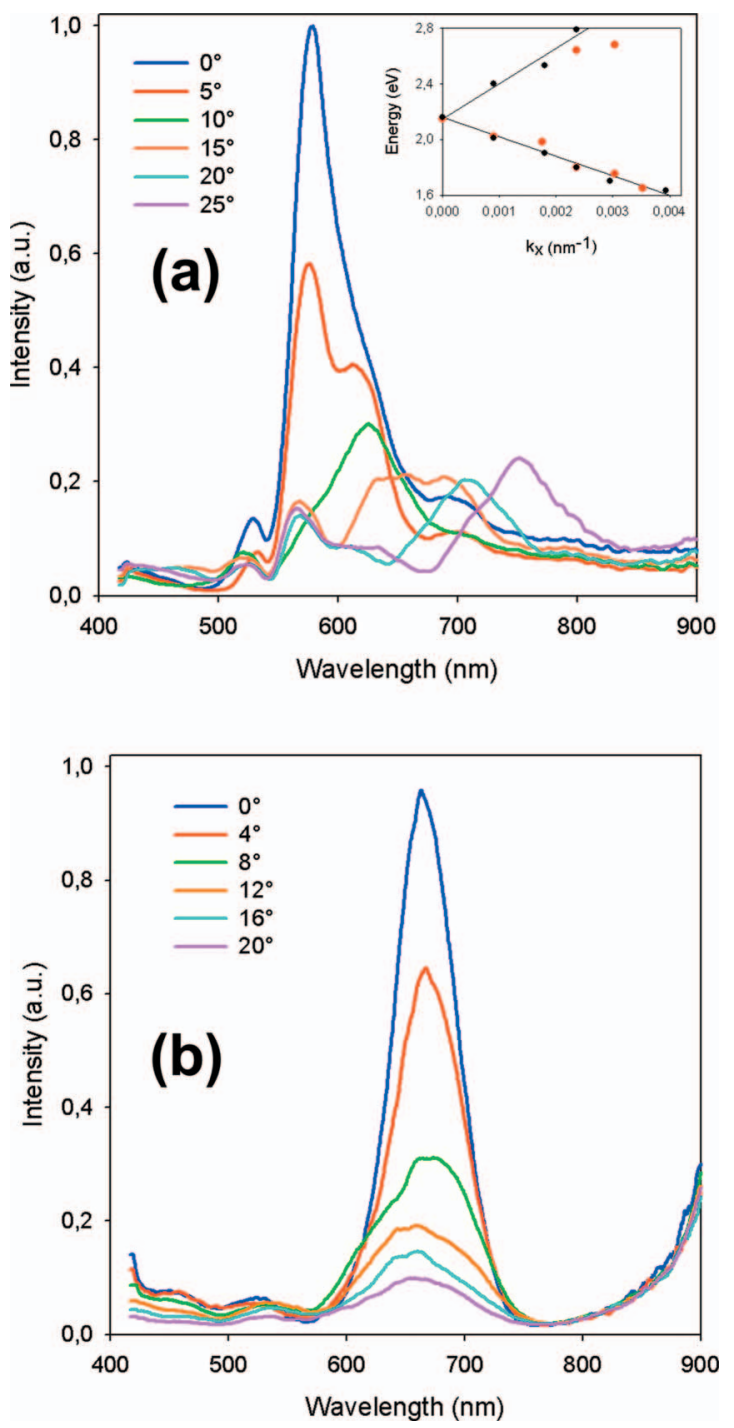

FIG. 48. (Color) Collimation of light emerging from a single aperture. (a) Transmission spectra at $p$-polarized normal incidence for a single slit flanked at the output surface by two periodic arrays of five grooves (one placed to the left and the other to the right of the slit). The width of the slit and grooves is $40 \mathrm{~nm}$, the groove periodicity is $d=500 \mathrm{~nm}$, and the metal thickness is $h=300 \mathrm{~nm}$. The nominal depth of the grooves is $60 \mathrm{~nm}$. The spectra are recorded at various collection angles from $0^{\circ}$ to $25^{\circ}$. The inset shows a dispersion curve of the transmission peak locations versus parallel momentum. (b) Transmission spectra for the bull's eye geometry: a 250-nm-diameter cylindrical hole is milled into a $300-\mathrm{nm}$-thick silver film. It is surrounded by five circular trenches with depths of $60 \mathrm{~nm}$ and the groove periodicity is $600 \mathrm{~nm}$. The tail above $800 \mathrm{~nm}$ is an experimental artifact. From Lezec et al., 2002.

mitted terahertz wave form. A posterior development demonstrated that by varying the phase of the surface corrugation relative to the central aperture, dramatic changes can be made in the transmission resonance line shape (Cao et al., 2005). Beaming effects at terahertz frequencies were studied by Agrawal and Nahata (2006). It was shown how each groove produces a time-delayed replica of the total terahertz pulse that is evanescently 
coupled through the subwavelength aperture. These replicas are coherently superposed on each other and temporally shifted from one another in accordance with the spatial distance between grooves.

There have been several studies dedicated to improving both the enhanced transmission and beaming capabilities of the basic structures discovered in 2002. Ishihara, Hatakoshi, et al. (2005) and Ishihara, Ikari, et al. (2005) demonstrated that the inclusion of a Bragg reflector into the structure further increases the enhanced transmission through the bull's eye geometry if this contains a small number of rings. The same group recently showed (Ishihara et al., 2006) that by changing the shape of the aperture from a circular to a bow-tie one, not only the transmittance is largely increased but the spatial resolution of this structure is of the order of $\lambda / 17$. On the other hand, Caglayan et al. (2006b) experimentally tested the beaming capabilities of annular apertures surrounded by periodic arrays of concentric grooves, showing that the angular confinement can be as strong as $3^{\circ}$. Another important improvement on the transmissivity of these structures is associated with the use of sharpapex shape for the grooves in the periodic grating (Ishi, Fujikata, and Ohashi, 2005). Fabricated samples with this type of gratings show greater transmission than that of samples with rectangular grooves. Also, a modulation of the groove depth along the periodic array of indentations (Shi et al., 2007) can lead to a better confinement of the light emerging from the subwavelength aperture.

Resonant transmission and collimation of light can be achieved for two different wavelengths when the single aperture is surrounded by asymmetric configurations of periodic arrays of indentations (Bravo-Abad et al., 2003). The generation of off-axis directional beaming of light by a single subwavelength slit perforated on a metal film has also been recently proposed (Kim et al., 2007; Lin et al., 2007). The combination of single apertures perforated on a metal film with dielectric surface gratings [as proposed by Lin et al. (2006)] seems to offer a better performance than the one obtained when only metallic gratings are considered. Along this line, it has been shown that the dielectric properties of the surface surrounding the aperture can be modulated using metallic heterostrutures constructed with aluminum and silver (Wang and Wang, 2006; Nikitin, Brucoli, et al., 2008). Recently $\mathrm{Li}$ and colleagues (Li et al., 2008) proposed an improved structure by surrounding the central aperture with nonuniform and nonperiodic goves. In this way, the amplitude and phase of the power flowing from each groove can be adjusted in order to enhance the final transmission.

The theoretical modeling of both enhanced transmission and beaming effect in single apertures has confirmed that surface EM modes excited at the corrugated metal surface(s) play a key role in the emergence of both phenomena (Garcia-Vidal, Lezec, et al., 2003; MartinMoreno et al., 2003). This physical explanation has been corroborated and refined by other theoretical approaches and numerical investigations (Thomas and

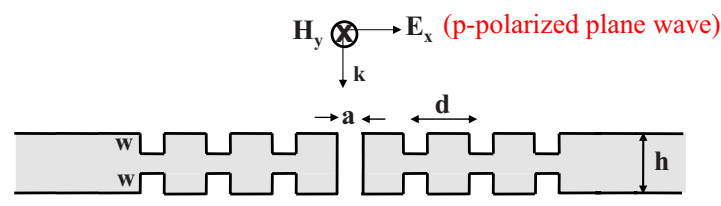

FIG. 49. (Color online) Schematic of a single slit of width $a$ in a metallic film of thickness $h$. This single slit is surrounded symmetrically by $2 N$ grooves (of width $a$ and depth $w$ ) in both input and output surfaces. The structure is illuminated by a normal incident $p$-polarized plane wave.

Hughes, 2004; Yu et al., 2005; Wang, Du, and Luo, 2006; Wang, Du, Lv, and Luo, 2006).

An interesting spin off of EOT and beaming effects in single apertures surrounded by periodic corrugations has been the appearance of both phenomena in socalled photonic crystals. As stated, the main ingredient necessary to observe both phenomena is the excitation of surface EM modes. Depending on the truncation of its surface, a photonic crystal may support a surface EM mode lying within the photonic band gap of the material. The emergence of both phenomena in photonic crystal waveguides was theoretically predicted by Moreno, Garcia-Vidal, and Martin-Moreno (2004). In a parallel development, Kramper et al. (2004) reported the emergence of directional beams with very low divergence angles from photonic crystal waveguides of subwavelength width. They also identified the key role played by the evanescent surface EM modes in achieving the low divergence beams. Several works have subsequently been devoted to analyze different aspects of EOT and beaming effects in photonic crystals. The first experimental study on the enhanced transmission assisted by surface EM modes in photonic crystals was by Bulu et al. (2005). Further theoretical investigations of those two effects (Moreno, Martin-Moreno, and GarciaVidal, 2004; Frei et al., 2005; Morrison and Kivshar, 2005; Tang et al., 2006) focused on the optimization of the surface geometry surrounding the waveguide in order to enhance both the transmission and collimation of the emerging beam. Two recent experimental reports have focused on improving the directionality of the transmitted beam by tuning the number of grating layers (Moussa et al., 2007) and to enlarge the operation bandwidth of the beaming effect by playing with the phase of the multiple beams emitted out of the surface of the photonic crystal (Li et al., 2007).

\section{B. Coupled-mode analysis}

In what follows we use the coupled-mode method to analyze from a fundamental point of view these two phenomena (enhanced transmission and beaming) for the basic $1 \mathrm{D}$ structure, a single slit surrounded by a finite array of grooves (see Fig. 49). We mention here that the physical origin of the resonant phenomena in single apertures for 1D and 2D structures is similar, as shown by Chang et al. (2006). 


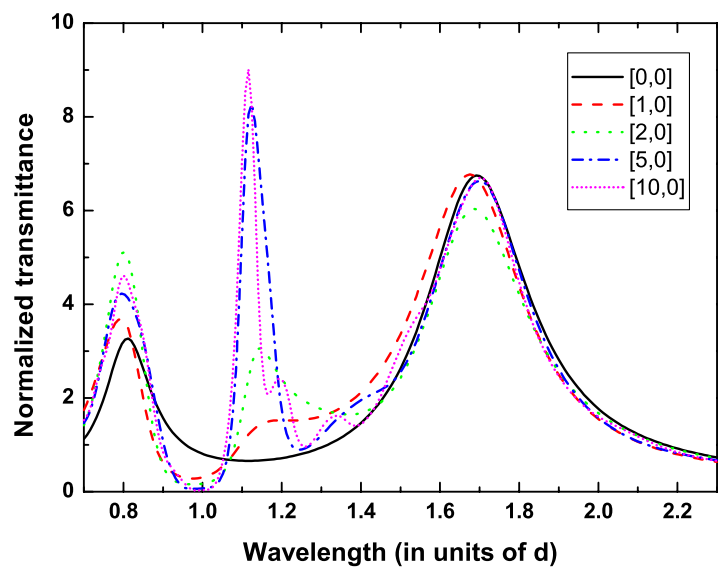

FIG. 50. (Color online) Normalized-to-area transmittance as a function of the number of grooves when only the input surface is corrugated. The width of the slit and grooves is $a=0.08 d$, the depth of the grooves is $w=0.2 d$, and the metal thickness is $h$ $=0.7 \mathrm{~d}$. The notation $[N, 0]$ means that $2 \mathrm{~N}$ grooves are perforated in the input surface and 0 in the exit surface. From Garcia-Vidal, Lezec, et al., 2003.

In order to simplify the modeling as much as possible, we consider that the metal is a PEC and take into account only the TEM modes inside the central slit and grooves.

First, we analyze the influence of patterning the input surface on the total transmittance through the structure. Figure 50 shows the dependence of the normalized-toarea transmittance $T(\lambda)$ with the number of grooves. The notation $[N, 0]$ means that $2 N$ grooves are placed symmetrically to the left and right of the central slit at the input surface, whereas the output surface presents no corrugation. As in previous cases, the period of the array $d$ is used as the unit length of the structure. The curve for $N=0$ corresponds to the single slit case. In this wavelength regime $T(\lambda)$ presents two maxima whose origin is related to the excitation of Fabry-Perot resonances inside the slit, as discussed in Sec. II.A. Figure 50 also shows that a maximum in $T(\lambda)$ develops at $\lambda$ $\approx 1.1 d$ as the number of grooves is increased. The enhancement factor for $N=10$ is around 9 and saturates for $N \geqslant 10$ for this particular set of geometrical parameters (Garcia-Vidal, Lezec, et al., 2003). Note also that the transmission peaks associated with the excitation of Fabry-Perot resonances of the single slit remain almost unaltered when grooves are placed at the input surface.

Figure 51 shows that the output corrugation has little effect on the total transmittance. Corrugating the output surface induces a change in $T(\lambda)$ of, at most, some $20 \%$ at the resonant wavelength. Therefore, in order to understand the features appearing in the total transmittance, we concentrate on the analysis of the structure in which the single slit is surrounded by a finite array of indentations at the entrance surface. In this case, the general system of equations (1) can be further simplified to a set of $2 N+2$ equations, $2 N$ being the number of grooves:

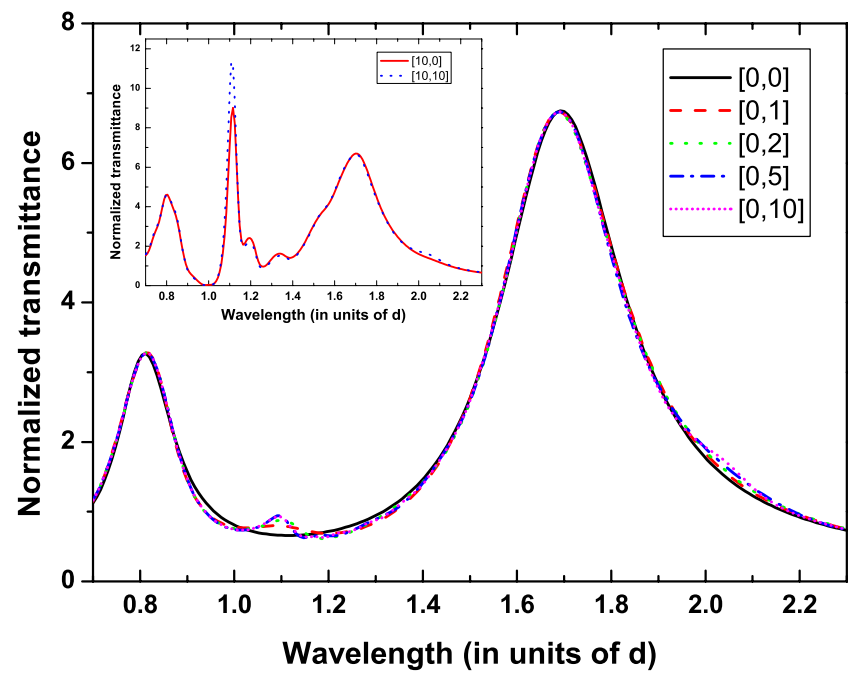

FIG. 51. (Color online) Normalized-to-area transmittance as a function of the number of grooves when only the exit surface of the slit is corrugated. As in Fig. 50, the width of the slit and grooves is $a=0.08 d$, the depth of the grooves is $w=0.2 d$, and the metal thickness is $h=0.7 d$. The notation $[0, N]$ means that $2 N$ grooves are milled at the exit surface and 0 at the input surface. The inset shows the transmittance for the $[10,0]$ and [10, 10] configurations. From Garcia-Vidal, Lezec, et al., 2003.

$$
\begin{aligned}
& \left(G_{\alpha \alpha}-\Sigma_{\alpha}\right) E_{\alpha}+\sum_{\beta \neq \alpha} G_{\alpha \beta} E_{\beta}-G_{\alpha}^{V} E_{\alpha}^{\prime} \delta_{\alpha 0}=I_{\alpha}, \\
& \left(G_{00}-\Sigma_{0}\right) E_{0}^{\prime}-G_{0}^{V} E_{0}=0 .
\end{aligned}
$$

From these equations, it is possible to identify two mechanisms for enhancing $T(\lambda)$ (in addition to the Fabry-Perot resonances already emerging in a single isolated slit). The excitation of groove cavity modes provides large $E_{\alpha}$ at the resonant condition $G_{\alpha \alpha}-\Sigma_{\alpha} \approx 0$. For the case of very narrow grooves, this condition is fulfilled for $\lambda=4 w /(2 n+1)$, with $n$ as an integer. From Eqs. (30) it is clear that large $E_{\alpha}$ can provide extra illumination into the central slit. However, this reillumination process at the central slit depends on a weighted sum over all $E_{\alpha}$, so the phases of the different contributions coming from the grooves must be carefully taken into account. As explained, in a corrugated PEC [see Eq. (A18)] the coupling between the grooves and the central slit $G_{\alpha 0}$ is governed by the Hankel function $H_{0}^{(1)}(x)$. From the asymptotic behavior of $H_{0}^{(1)}$ for large $x$, $G_{\alpha 0}$ is proportional to $\exp \left(i k_{\omega} d|\alpha|\right)$. Therefore, all light that is reemitted by the grooves reaches the central slit in phase for $\lambda \approx d$. The extra peak in transmittance appearing at $\lambda$ close to $d$ is due to the combination of these two mechanisms: groove cavity mode excitation and inphase groove reemission.

The theoretical explanation based on those two mechanisms can be further elaborated by invoking the formation of a geometrically induced surface EM mode, appearing when the surface of a semi-infinite PEC is perforated by a periodic array of $1 \mathrm{D}$ grooves. As in the case of a $1 \mathrm{D}$ array of slits, the dispersion relation of the 


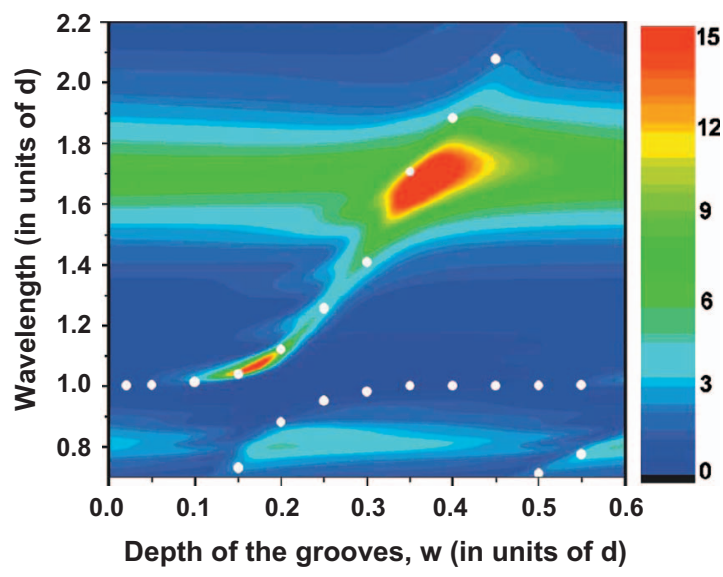

FIG. 52. (Color) Contour plot of the transmittance versus groove depth $(w)$ and wavelength (both in units of $d$ ) when a central slit is flanked symmetrically by a total of 20 grooves. Here $a=0.08 d$ and the thickness of the metal $h=0.7 d$. White dots correspond to the spectral locations of the surface EM modes of an infinite array of rectangular grooves with varying $w$ as calculated from Eq. (A28) with $k_{x}=2 \pi / d$. From GarciaVidal, Lezec, et al., 2003.

surface EM modes supported by an infinitely periodic array of grooves can be calculated by neglecting diffraction effects [Eq. (A28)]. These surface EM modes may become leaky when diffraction effects are considered. However, as a first approximation, the spectral locations of the surface EM modes, which can be excited by a normal incident $p$-polarized plane wave, can be calculated from Eq. (A28) by imposing $k_{x}=2 \pi / d$. Figure 52 shows the close correspondence between the spectral locations of the transmission resonances and the surface EM modes for an infinite array of rectangular grooves. Figure 52 also shows that these surface EM modes originate from the interplay between the groove cavity mode $\left[\tan k_{\omega} w \rightarrow \infty\right.$ in Eq. (A28)] and the in-phase groove reemission mechanism [in the limit $w \rightarrow 0, k_{x} \rightarrow k_{\omega}$ in Eq. (A28)].

The excitation of a surface EM mode is accompanied by a large enhancement of the $E$ field inside the grooves (see Fig. 53), whose reemission into the central slit leads to an enhancement in the transmittance. Therefore, this surface EM mode acts as a funnel that collects the light

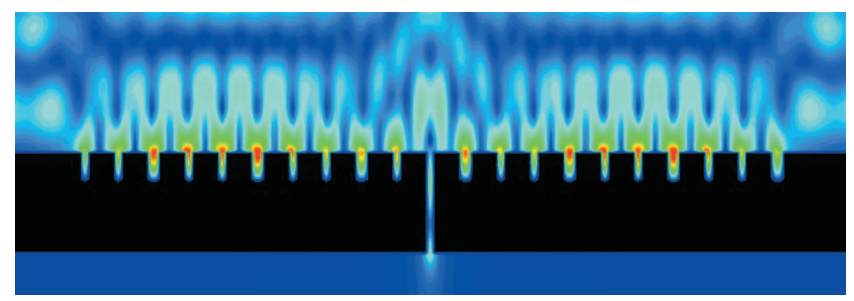

FIG. 53. (Color) Electric field amplitude associated with the transmission peak $\lambda=1.1 d$ emerging when a single slit is flanked by a total of 20 grooves, placed symmetrically at its entrance surface. The geometrical parameters of the structure are as in Fig. 50. impinging at the area surrounding the slit and is able to redirect it into the slit area.

We now use the coupled-mode method to analyze the beaming phenomenon for the 1D case: a single slit flanked by a periodic array of grooves. As shown before, the angular distribution of the transmitted light depends only on the properties of the output surface. Therefore, we can focus on a simplified geometry, composed by a single slit (driven by a wave of amplitude $A_{0}$ ) and surrounded by a finite array of grooves at its exit surface. Within both the single TEM mode and PEC approximations, the set of $2 N+1$ linear equations governing the modal expansion amplitudes of the $E$ field at the exit side $E_{\alpha}^{\prime}$ is

$$
\left(G_{\alpha \alpha}-\Sigma_{\alpha}\right) E_{\alpha}^{\prime}+\sum_{\beta \neq \alpha} G_{\alpha \beta} E_{\beta}^{\prime}=2 i A_{0} \delta_{\alpha 0} .
$$

In terms of these amplitudes, the $y$ component of the magnetic field in the transmission region is given by

$$
H_{y}(\vec{r})=\frac{1}{\mu_{0} c} \sum_{\alpha} E_{\alpha}^{\prime} G(\alpha, \vec{r}),
$$

where the propagator that connects indentation $\alpha$ with point $\vec{r}, G(\alpha, \vec{r})$, is also related to $H_{0}^{(1)}$ :

$$
G(\alpha, \vec{r})=\frac{i k_{\omega}}{2 \sqrt{a}} \int_{x_{\alpha}-a / 2}^{x_{\alpha}+a / 2} H_{0}^{(1)}\left(k_{\omega}\left|x \vec{u}_{x}-\vec{r}\right|\right) d x,
$$

where $x_{\alpha}$ is the $x$ coordinate of the center of indentation $\alpha$.

For the polarization we are considering ( $p$-polarized light), the other components of the EM fields can be obtained from $H_{y}(\vec{r})$, therefore allowing the computation of the radial component of the Poynting vector $S_{r}(\theta, \lambda)$ and the far-field angular distribution of the transmitted radiation $I(\theta, \lambda)=r S_{r}(\theta, \lambda) / T(\lambda)$, where $r \gg \lambda$. Note that this angular distribution is normalized to the total transmittance $T(\lambda)$. In this way, $I(\theta, \lambda)$ $=1 / \pi$ for a single slit perforated on a PEC.

As Eq. (32) shows, the system under consideration behaves like an equivalent diffraction grating, in which the EM field amplitudes at the emitters present a strong dependence on wavelength and on the distance to the central slit, as shown below. Figure 54(a) shows $I(\theta, \lambda)$ for very shallow grooves, $w=0.02 d$. In this case the angular transmission profile is well described by a diffractionlike first-order approximation: as $\Sigma_{\alpha} \gg 1$ (for $\alpha \neq 0$ ), we obtain $E_{\alpha}^{\prime} \approx-G_{\alpha 0} E_{0}^{\prime} /\left(G_{\alpha \alpha}-\Sigma_{\alpha}\right)$ and for the central slit $E_{0}^{\prime}$ $\approx 2 i A_{0} /\left(G_{00}-\Sigma_{0}\right)$, so $\left|E_{\alpha}^{\prime}\right| \ll\left|E_{0}^{\prime}\right|$. From the asymptotic expansion of $H_{0}^{(1)}\left(k_{w} x\right),\left|E_{\alpha}^{\prime}\right| \propto \alpha^{-1 / 2}$, while the phase of $E_{\alpha}^{\prime}$ is $\phi_{\alpha}=k_{w} d|\alpha|+\phi$ for groove $\alpha$ and a value $\phi_{0}$, not following the previous law, for the central slit. As the phase of the $E$ field depends linearly with the distance to the slit, this phase can be canceled in the far-field region by the phase accumulated in the optical path at angles $\theta^{(m, \pm)}(\lambda)=\arcsin (m \lambda / d \pm 1)$ for integer $m$. This is the origin of the weak beaming observed at those angles in Fig. 54(a). 

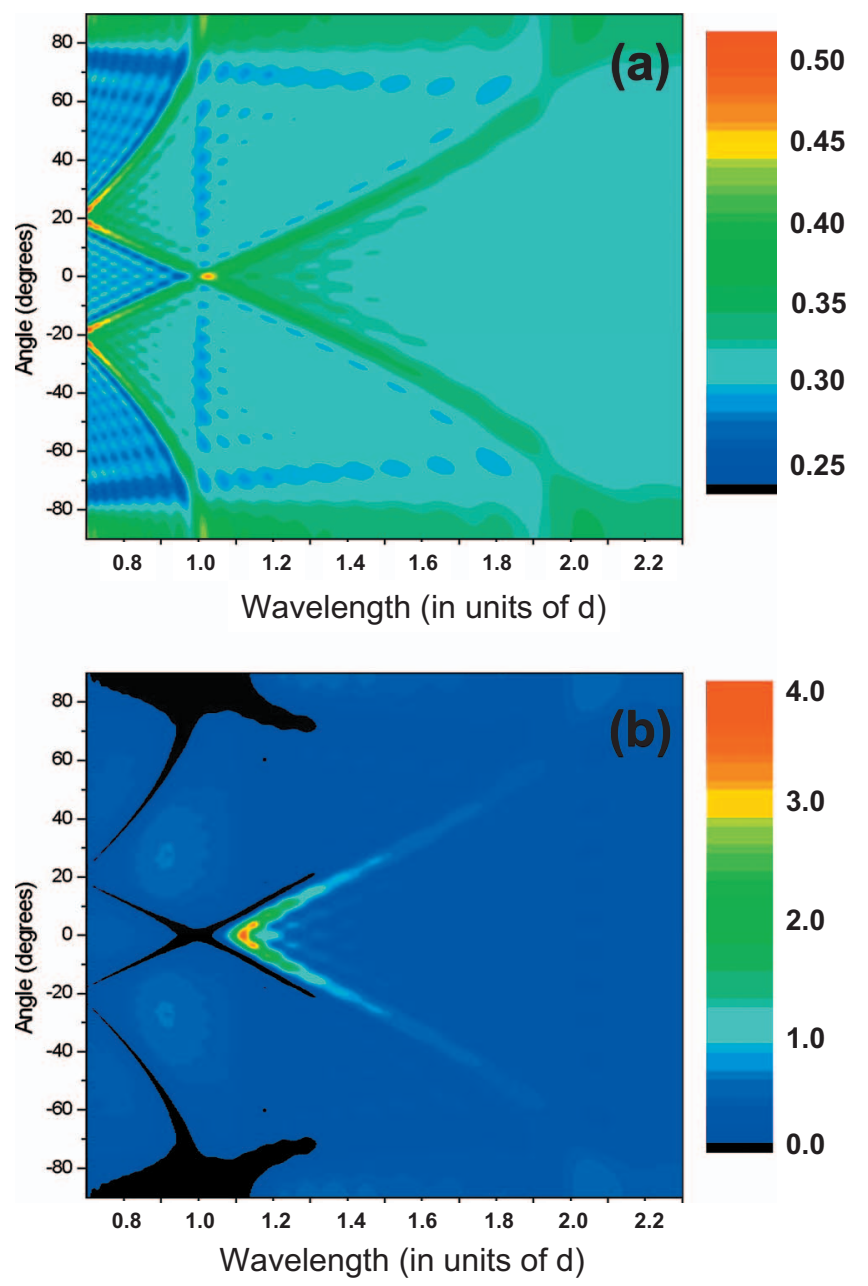

FIG. 54. (Color) Contour plots for $I(\theta, \lambda)$ (see the text) for two different groove depths: (a) $w=0.02 d$ and (b) $w=0.2 d$. The width of the central slit and grooves is $a=0.2 d$. The total number of grooves that are placed symmetrically with respect to the central slit is 20. From Martin-Moreno et al., 2003.

For deeper grooves the first-order approximation starts to fail. Actually, for some values of the parameters, the iterative solution of Eq. (31) does not converge, which is a signature of the building up of an eigenmode of the system (Martin-Moreno et al., 2003). Indeed, close to the groove cavity mode resonance condition $\operatorname{Re}\left(G_{\alpha \alpha}-\Sigma_{\alpha}\right)=0$ we find the formation of a collective surface EM mode, characterized by a large increase of $E_{\alpha}^{\prime}$. The dispersion relation of the surface EM modes for the case of an infinite array of grooves is given by Eq. (A28). This resonance leads to a more pronounced beaming phenomenon, as shown in Fig. 54(b). This interpretation of the beaming phenomenon in terms of the excitation of leaky surface EM modes is also supported by the coincidence of the resonant wavelength in which beaming phenomenon at $0^{\circ}$ occurs with the wavelength of maximum transmittance (when the same corrugation is placed at the input surface).

Associated with the beaming effect, there is also a focusing effect, as shown in Fig. 55. This figure shows the $E$-field amplitude in real space evaluated at the resonant

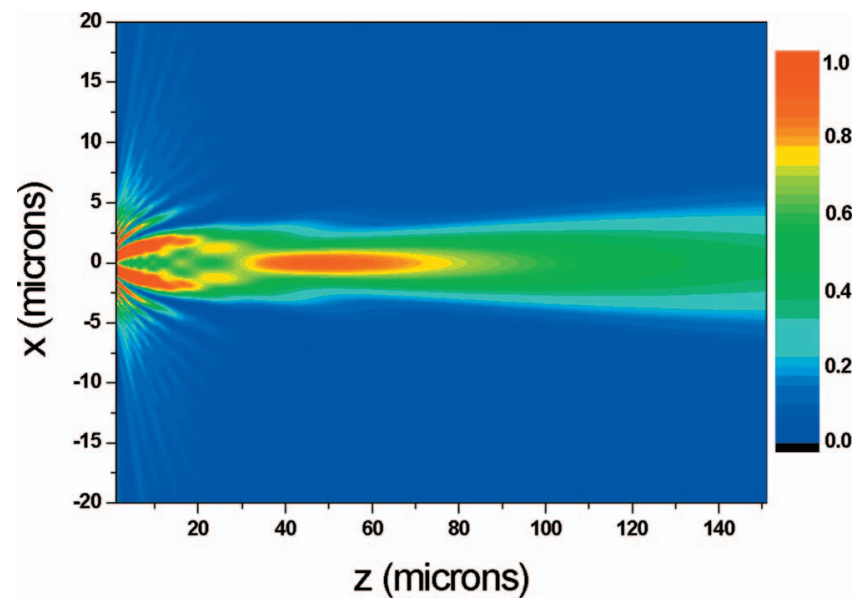

FIG. 55. (Color) Electric field amplitude profile (in arbitrary units) as a function of $x$ and $z$ for the case $N=10$ with $a$ $=0.08 d$ and $w=0.17 d$ that corresponds to the optimal parameters for beaming. In this case $d$ is chosen to be $500 \mathrm{~nm}$, as in the experimental samples discussed in Sec. IV.A. This $E$-field profile is evaluated at the resonant wavelength, $\lambda_{R}=1.06 \mathrm{~d}$. From Garcia-Vidal, Martin-Moreno, et al., 2003.

wavelength for the case $N=10$. A very elongated focus emerges in the intermediate field regime between the near and far fields. As in the case of beaming, this lensing ability (focal depth, length, and width) is controlled by the output corrugation (Garcia-Vidal, MartinMoreno, et al., 2003).

\section{APPLICATIONS}

Before the EOT phenomenon was reported in 1998, subwavelength apertures were commonly seen as suffering from both poor transmission and strong diffraction. For instance, for scanning near-field optical microscopy, the aperture provided the subwavelength resolution but was also the source of the low signal intensity, typically explained with Bethe's theory (Betzig and Trautman, 1992). For the past decade, the potential applications of subwavelength apertures have been revisited with the new understanding that the EM fields can be strongly enhanced at the apertures in the metal film. The interest has been further stimulated by the high contrast provided by these structures combined with the possibility of tailoring their properties by sculpting the metal surface. The two areas that have been the object of numerous studies are molecular sensing and spectroscopy and stand-alone photonic devices. These applications will be presented in greater detail below.

There are, however, general practical considerations that need to be taken into account when designing aperture structures where the choice of structural parameters and materials must be optimized for a given application. In what follows, we summarize the main fundamental results obtained during the last ten years of EOT research (and discussed in part in previous sections) that have important implications when thinking of possible applications of the EOT phenomenon. Metal films with 
apertures are robust and can easily be fabricated by standard techniques such as $e$-beam or focused-ion beam lithography. At the wavelength of interest, the real part of the dielectric constant of the metal must be negative and its absolute value must be larger than that of the dielectric material in contact with the metal. This is a basic criteria for the SPP to exist at the metal-dielectric interface. At the same time, the imaginary part of the metal-dielectric constant should be as small as possible to avoid damping by absorption. For instance, $\mathrm{Ag}$ is good throughout the visible and near-ir part of the spectrum, while $\mathrm{Au}$ only works at wavelengths longer than the interband transitions around $550 \mathrm{~nm}$. In the uv, Al is a suitable metal. The quality of the metal film is also important. It should be as smooth as possible to avoid unwanted scattering events. The film thickness should be such that it is opaque at the wavelength of interest, e.g., approximately ten skin depths.

The resonant wavelengths can be most easily tuned by varying the periodicity and the symmetry of the hole arrays or the periodic features surrounding individual apertures, as discussed in Secs. III and IV. The individual hole shape and dimension relative to the resonant wavelength will also affect the transmission mainly through its cutoff function. If high absolute transmissions are needed, large apertures should be used although there will be a tradeoff as that resonance will become broader. If, on the other hand, high surface field intensities are desired for sensing or nonlinear affects, then the hole dimensions relative to the array should be reduced to near or below the cutoff of the aperture. Apertures such as slits give rise to Fabry-Perot resonances, which further enriches the transmission spectrum. When single apertures are surrounded by periodic features such as grooves, then the profile of the periodic features (width, depth or height, shape) must be adjusted to obtain optimal transmission. In this case the distance of the periodic structure from the aperture will also influence the outcome through a phase shift. Since SPP modes are supported by both interfaces of the metallic film, maximum transmission is obtained when the energies of these modes coincide, and therefore when the refractive indices of the dielectric material in contact with the metal on both sides are the same. At the same time the modes can couple with the apertures in the array, giving rise to new mode energies and broadening. Such coupling decreases with film thickness or hole depth. Sometimes a thin binding metal, typically $\mathrm{Cr}$ or $\mathrm{Ti}$, is deposited between a substrate and the metallic film to improve the adherence of the latter. This can completely dampen the SPP modes at that interface when the binding metal has a high imaginary dielectric constant.

The finite size of the hole array can affect the transmission quality (peak width and intensity), as discussed in Sec. III.B.6. For optimal transmission, the array should be larger than the propagation length of the SPP on the array. This SPP propagation length is much smaller than on a smooth film, e.g., in the visible it is typically one order of magnitude smaller. Finally, when trying to characterize the optical properties of a given sample, it is important to use a collimated beam on the input side. This gives a well-defined wave vector which facilitates the interpretation of the transmission whether one is simply determining the spectrum or the dispersion curve of the structure.

\section{A. Molecular sensing and spectroscopy}

Surface plasmons have played a major role in sensing and molecular spectroscopy for the past 30 years, for instance, in the detection of small refractive index variations by the technique known as surface plasmon resonance (SPR) (Nylander et al., 1982) and in surfaceenhanced Raman spectroscopy (SERS) (Jeanmaire and Van Duyne, 1977). With this in mind and considering the involvement of SPPs in the EOT process, it is not surprising that aperture structures have been extensively explored for spectroscopic purposes. In addition, aperture structures can be added easily to standard equipment. Since applications in this area have already been the subject of detailed reviews (Coe et al., 2006, 2008; Gordon et al., 2008; Sinton et al., 2008), only representative studies will be outlined here. Aperture structures have been used for molecular absorption, fluorescence, vibrational spectroscopy (ir and Raman), and SPR.

\section{SPR}

In traditional SPR, minute refractive index variations are measured at the interface of a translucent metal film placed on a prism. A light beam impinges on the back of the metal film and is mostly reflected except at an angle or wavelength which allows evanescent coupling to SPPs on the opposite side. This coupling condition is very sensitive to the local index and hence any molecular process at the surface that results in a net index variation can be detected. The transmission of hole arrays are also sensitive to the refractive index at the metal surface (Krishnan et al., 2001), and the binding of biomolecules can be followed by simply measuring a shift in the transmission peaks under a microscope (Brolo, Gordon, et al., 2004; Liu and Blair, 2004). The simplicity of the setup was, however, offset by low sensitivity due to the broadness of the peaks. Further developments have brought the sensitivity to a level where this approach is competitive with existing commercial SPR apparatuses (Stark et al., 2005; Tetz et al., 2006). For instance, by adding two polarizers at $90^{\circ}$ in the path of the transmitted beam, the hole array transmission peak becomes so narrow that refractive index variations smaller than $10^{-5}$ can be detected (Tetz et al., 2006). Isolated holes as well as disordered patterns of holes have also been used, demonstrating the various approaches that can be tried for SPR sensing (Dahlin et al., 2005; Rindzevicius et al., 2005; Gao et al., 2007).

\section{Enhanced absorption}

The use of hole arrays to measure molecular vibrational spectra illustrates well the potential of metallic aperture structures for spectroscopy. In 2003, Coe and 
colleagues reported that an array tuned to the ir with transmission resonances around $1000 \mathrm{~cm}^{-1}$ could enhance the molecular absorption by at least two orders of magnitude (Williams et al., 2003). The free-standing arrays covered with a molecular monolayer were simply placed in the beam of a commercial Fourier transform infrared spectroscope and the transmission spectra recorded. Except for some spectral deconvolution, the approach is simple and allows one to follow chemical processes such as catalysis (Coe et al., 2006). The enhancement is related to the lifetime of the SPP or the SPP propagation length on the array's surface, which increases the absorption probability by the molecules. In the visible, the absorption enhancement is smaller (factor of $\sim 10$ ) due to the reduced SPP propagation length (Dintinger, Klein, and Ebbesen, 2006). Nevertheless, because the incident beam is reconstructed at the exit of hole array, the latter can be easily used in pump probe to do time-resolved spectroscopy and explore, for instance, excited state properties of very thin molecular layers or SPP-molecule interactions (Dintinger, Robel, et al., 2006). SERS has also been investigated on hole arrays (Brolo, Gordon, et al., 2004; Reilly and Rowlen, 2004; Lesuffleur, Kumar, Brolo, et al., 2007; Reilly et al., 2007). A quantitative analysis of the Raman signal intensity from a nonresonant probe at monolayer concentrations reveals that out of the $6 \times 10^{7}$ observed enhancement factor, $10^{5}$ is the result of the $\mathrm{Ag}$ film roughness whereas 600 is associated with the SPP modes at the apertures (Reilly et al., 2007). These values are much smaller than those obtained from random metallic films. However, the hole arrays have the advantage of being tunable, reproducible, and, under optimal design, capable of partially filtering out the incident beam while transmitting, for instance, the Raman-Stokes lines.

\section{Fluorescence}

Considering the absorption enhancement discussed above, it is only natural to expect that fluorescence can also be boosted. Indeed this has been demonstrated using both single and arrays of apertures (Levene et al., 2003; Liu and Blair, 2003; Brolo et al., 2005, 2006; Rignault et al., 2005). To analyze the enhancement, several factors have to be considered. The fluorescence can be increased due to the enhancement of the excitation rate but the radiative lifetime and the fluorescence quantum yield can also be modified. For instance, the fluorescence is quenched at short distances from the metal surface $(<20 \mathrm{~nm})$ and can be boosted at longer distances (Drexhage, 1974; Barnes, 1998). Finally, the environment can modify the radiation pattern of the fluorophore. Fluorescence correlation spectroscopy can particularly benefit from the use of a subwavelength aperture (Levene et al., 2003; Edel et al., 2005; Rignault et al., 2005; Wenger et al., 2008). The analyzed volume is reduced by $10^{3}$ as compared to using the focal point of a laser beam, which in turn allows the characterization of molecular fluorescence statistics at physiological concentrations (Blom et al., 2006). Both the excitation and emission can be enhanced due to an increase in the local density of states at the aperture (Wenger et al., 2008). Small apertures in metal films also have the advantage of facilitating parallel assays with multiple aperture structures on a given substrate. Note that in such a case, care must be taken to avoid cross-talk between the apertures since each one can launch SPPs. The possibility of structuring the metal surface around the aperture to increase the excitation intensity and beam the emission toward the photodetector should also be explored (Lezec et al., 2002).

Aperture structures also lend themselves well for integration in microfluidic systems (Sinton et al., 2008), where they can add label-free sensor capacity (De Leebeeck et al., 2007; Ji et al., 2008; Lesuffleur et al., 2008; Sharpe et al., 2008). The combination of optical contrast and simplicity of the transmission mode is particularly suited for analyzing rapidly small volumes at a given location in the high throughput of such systems. In addition, the ability to tailor the SPP resonance (wavelength, polarization sensitivity, etc.) to obtain signal enhancements should enable the measurement of various spectroscopic signals and boost the screening capacity as the analyte progresses in the channels. Finally, optical trapping by single holes or hole arrays could create new opportunities for on-chip integration and sensing (Sinton et al., 2008).

\section{B. Photonic devices and methods}

Perhaps the simplest application of aperture structures is as filters and polarizing elements (Genet and Ebbesen, 2007). Periodic arrays have well-defined resonances that can be tuned with geometrical parameters (lattice symmetry, period, and hole shape) and that can be changed by simply changing the relative angle of the filter to the incident beam. Such structures can also be made to act as polarizing filters by lowering the symmetry at the level of the holes and/or the arrays, as discussed in Secs. III.B.4 and III.B.7. Appropriately designed aperture structures can also act as wave plates to change the state of polarization of light (Drezet et al., 2008). On the other hand, the large polarization anisotropy combined with the peak shifts induced by the high aspect ratios in arrays of rectangular holes (as discussed in Sec. III.B.4) can be exploited to generate structures of which the color of the emitted light can be tuned through a modulation of the polarization (DiMaio and Ballato, 2006). These color filters and polarization elements have the advantage of being robust to high laser powers as long as the metal absorption is negligible at the wavelength of interest.

Such features have stimulated quite a few studies to use aperture structures in lasers, in particular, in verticalcavity surface-emitting lasers (VCSELs) (Shinada et al., 2003; Hashizume and Koyama, 2004; Guo, Song, and Chen, 2007, 2008; Ohno et al., 2007; Onishi et al., 2007). Recently the beaming effect associated with the corrugation placed on the output surface surrounding the aperture has been used to extract collimated beams in 


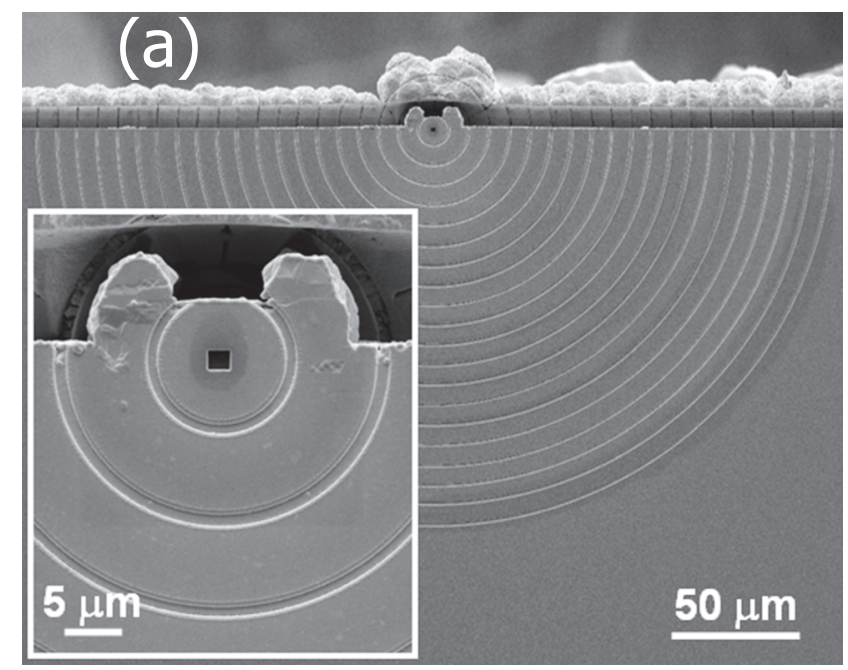

Intensity (normalized)

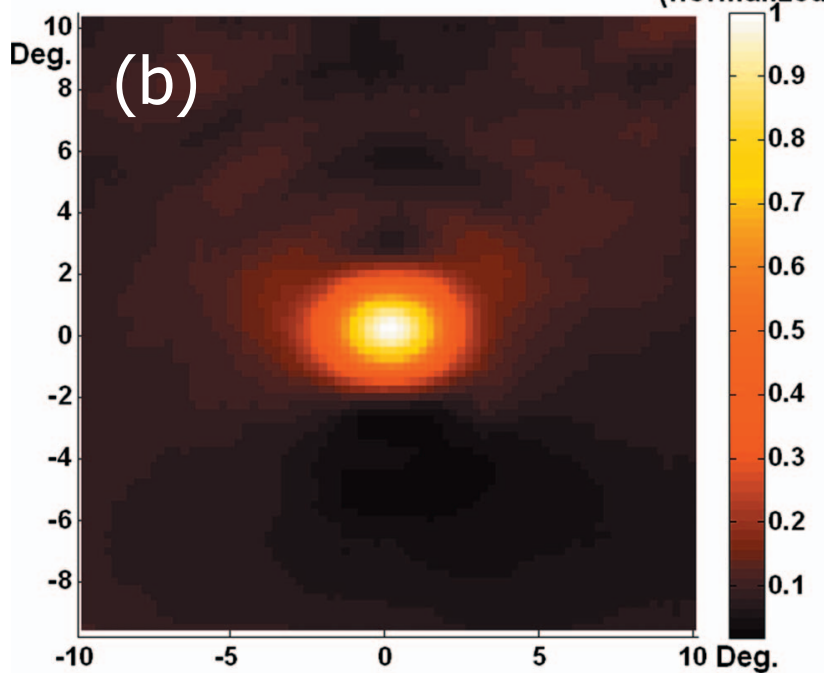

FIG. 56. (Color) Plasmonic collimator for a quantum cascade laser. (a) A SEM micrograph of the quantum cascade facet patterned with 20 rings. The rings are 0.6 and $1.0 \mu \mathrm{m}$ deep with a periodicity of $7.8 \mu \mathrm{m}$. The inset is a zoom-in view of the facet. (b) Measured 2D far-field intensity distribution for the device. Courtesy of Nanfang Yu.

quantum cascade lasers (Yu, Blanchard, et al., 2008; Yu, Fan, et al., 2008). This idea has been checked in both 1D and $2 \mathrm{D}$ geometries. In the $2 \mathrm{D}$ case, the plasmonic collimator consists of a subwavelength square hole opened on a laser active region and an array of half rings centered on the aperture [see Fig. 56(a)]. The period of the rings $(7.8 \mu \mathrm{m})$ was designed to match the SPP wavelength of the patterned metal surface. The angular dependence of the measured far-field intensity emerging from the structure [see Fig. 56(b)] shows that the device has a divergence angle of around $3^{\circ}$, giving an antenna directivity number of $27 \mathrm{~dB}$. For the original unpatterned laser, this number was $8 \mathrm{~dB}$.

While single resonant apertures can provide a very small bright subwavelength laser beam, multiple apertures with polarization selectivity can stabilize and control the polarization output angle of VCSELs. As a con-

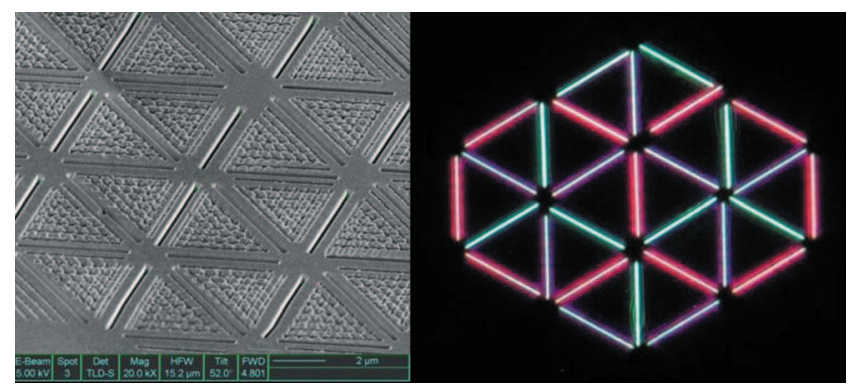

FIG. 57. (Color) Photon sorting based on EOT. Left: SEM image of a triangular lattice of slits fabricated by FIB in a $\mathrm{Au}$ film. Inside each triangle, gratings with different periods to couple light of different wavelengths are superimposed. Each grating selectively sends the resonating color to a slit where it is transmitted to the opposite side. Right: Far-field image of the light emerging from the structure on the left. Courtesy of Eric Laux.

sequence, several VCSELs on the same chip can be operated in parallel and coupled to one optical fiber opening new possibilities for polarization multiplexing (Hashizume and Koyama, 2007; Onishi et al., 2007). A considerable amount of light remains trapped in lightemitting diodes (LEDs), whether inorganic or organic, due to the high refractive index of the materials. In addition, conductive electrodes are necessary on both sides of the LEDs for current injection purposes, which can add to the problem. The light emitted by the diode can couple to SPPs in the electrode which are then damped in the metal unless scattered out and decoupled into freely propagating light. If an array of apertures is engraved in the electrodes, the electrode can still serve its original function and also provide an outcoupling mechanism of the SPPs and the trapped light as has been demonstrated for organic LEDs (Liu et al., 2004, 2005; Hsu et al., 2008). Such results are very promising and need to be tested for inorganic LEDs, which remain the major product on the market.

Just as photoemitters, photodetectors can also benefit from incorporating metallic apertures (Collin et al., 2003, 2004; Ishi, Fujikata, Makita, et al., 2005; Laux et al., 2008). Corrugated metal surfaces can act as resonant antennas to capture the incoming light, which can then be transmitted through one or more apertures to the photovoltaic elements. Smaller photovolatic elements can therefore be used to capture the same amount of light, which reduces the impedance of the device and thereby increases the operational speed. This should be particularly beneficial for silicon-based technology where such miniature photodetectors could be used for clocking purposes (Ishi, Fujikata, and Ohashi, 2005). Recently it was demonstrated that interdigitated metallic corrugations could also be used to separate different wavelengths of light impinging on the surface, which are then transmitted through individual apertures, as shown in Fig. 57. This photon-sorting capacity appears promising for spectral and polarimetric imaging (Laux et al., 2008).

Ever since it was shown that light could be beamed from a subwavelength aperture by texturing the output 
surface of the metal surrounding the opening (Lezec et al., 2002), the possibility of using such structures as novel kind of lenses and multiplexing devices has been the subject of many studies (Beruete, Campillo, et al., 2004; Caglayan et al., 2006a; Chang et al., 2006; Chen et al., 2006; Feng and Dawson, 2007; Hendry et al., 2007; Huang, Du, and Luo, 2007; Kim et al., 2007, 2008; Shi et al., 2007; Hendry, Garcia-Vidal, et al., 2008; Min et al., 2008). The profile of the beam emerging from the surface is sensitive to the various modes present and the scattering of the electromagnetic waves in the structure. Even quasiperiodic arrays can lead to focusing (Huang, Zheludev, et al., 2007). More generally, such control of the electromagnetic field profiles in the vicinity of subwavelength structures offers the possibility of designing a midfield microscope, which operates in the regime between the near field and the far field. Recently, the point-spread function (PSF) of such a microscope has been calculated and compared to that of a confocal microscope (Docter et al., 2007). It turns out that such a microscope has a PSF that is comparable to that of a confocal microscope. However, it can be engineered to have multiple spots, all with the same PSF, to greatly reduce measurement times. The first measurements of the PSF confirm the theoretical calculations (Docter, 2008).

The addition of all optical control by resonant excitation (Hendry, Garcia-Vidal, et al., 2008) or by addition of a nonlinear material (Min et al., 2008) allows for further beam control. More generally, it has been shown that the transmission through aperture arrays can be controlled optically using nonlinear materials (Porto et al., 2004; Wurtz et al., 2006) and molecular excited states (Dintinger, Robel, et al., 2006), electrically with liquid crystals (Kim et al., 1999; Dickson et al., 2008) and semiconductors (Shaner et al., 2007), and acoustically (Gérard et al., 2007). Such control is possible because the SPP resonances which give rise to the EOT are sensitive to the refractive index at the metal surface, which is modified in these processes. The transmission of hole arrays has also been switched directly by optical femtosecond pumping into the interband transitions of $\mathrm{Au}$ films (Halte et al., 2005), which modifies momentarily the dielectric function of the metal and thereby the SPP resonances. The transmission (filter) switching speeds that have been demonstrated reach terahertz frequencies using all optical control (Dintinger, Robel, et al., 2006).

The realization of surface plasmonic miniature photonic circuits will require, among other things, efficient surface plasmon launchers (Ebbesen et al., 2008). Hole arrays and individual apertures have been used to generate SPP beams at a metal surface (Devaux et al., 2003; Kim et al., 2003; Baudrion et al., 2008; Kihm et al., 2008). The shape of the SPP beam can be controlled by the disposition of the holes in the array (Laluet et al., 2007) or by texturing the metal surface near the aperture (López-Tejeira et al., 2007). The exact configuration will depend on the application but one of the advantages of the aperture structures is the coupling of light to SPPs via backside illumination thereby avoiding parasitic light and noise.

From the beginning it was quite clear that the EOT process had potential for achieving subwavelength lithography when one considered the subwavelength dimensions of the apertures and the high contrast the optically thick metal films provided. Such lithography mask would be activated by periodic corrugations with apertures only at desired locations. Naturally it will, however, only work in the near or proximate field. Such subdiffraction lithography with sub-100-nm features has been demonstrated using a variety of conditions (Alkaisi et al., 1999; Luo and Ishihara, 2004; Srituravanich et al., 2004; Schellenberg et al., 2005; Shao and Chen, 2005, 2008; Chang, Tsao, and Wei, 2007; Seo et al., 2007; Wei et al., 2007) and could be an alternative to the complex and costly modern projection lithographic techniques.

\section{CONCLUSIONS AND PERSPECTIVES}

It is clear that much insight has been gained over the past decade into the optics of subwavelength apertures in metal films. The contributions of various optical modes to the transmission process resulting in high local EM fields, which more than offset the attenuation by the cutoff function of the apertures, have been identified. The transmission can be tailored and optimized for a given need by an appropriate choice of geometrical and material parameters.

In a relatively short period of time, metallic aperture structures have found many applications as discussed. They are already used for research purposes in spectroscopy and sensing, and it is probably just a question of time before they find commercial use such as in analytical chips, where parallel data acquisition is critical. The demonstration of photonic devices enhanced by aperture structures shows promising routes for future research and development. In fact there is much ongoing effort in industry that is likely to lead to new products once the engineering issues have been resolved. For instance, inorganic LEDs could benefit from improved light extraction and aperture structures are a serious contender to achieve this if they can be successfully integrated in commercial devices. As detailed next, the aperture structures can still benefit from further fundamental studies and exploration, which will open new avenues.

Maximizing the electric fields at a given location on and/or off the surface can still benefit from further work. Shaping the electrical fields on multiple length scales is expected to be of significant interest for various applications. For instance, in cold atom research (Alloschery et al., 2006), for the simultaneous manipulation of a large number of optical traps, all aspects of light fields near subwavelength apertures will be important, as the traps have to be situated at a suitable distance from the metal surfaces to prevent heating of the atoms through an interaction with the Fermi electrons. In an alternative approach, it has been demonstrated theoretically that the EOT phenomenon can be applied to matter waves to 
manipulate cold atoms such as Bose-Einstein condensates (Moreno et al., 2005; Fernandez-Dominguez et al., 2006, 2008), which again would require tailoring the EM fields in and around the apertures.

Furthermore, the use of nonlinear interactions to switch the fields in the vicinity of the apertures will allow a high degree of spatiotemporal control of electrical fields, which is of both fundamental and applied interest. It is anticipated that coherent control, with or without learning algorithms, will play an important role to achieve the desired spatiotemporal control. By all optical switching of the transmission through subwavelength apertures, not only the amplitude but also polarization properties may be efficiently modified on femtosecond time scales thereby enabling an ultrafast control of electromagnetic fields. This will allow for not only timecontrolled filters, as already demonstrated at terahertz rates (Dintinger, Robel, et al., 2006), but also timedependent coupling to plasmonic circuitry (Ebbesen et al., 2008). More efforts in the texturing of the output surface around apertures could lead to miniature photonic devices with dynamic steering and multiplexing capacity.

The combination of several hole arrays can also lead to improved capabilities and new properties. Already cascading two hole arrays in the path of the light beam can lead to increased transmission as compared to a single array due to interlayer coupling (Ye and Zhang, 2005). The transmission can be very strong even when no direct line of sight exists between the apertures in the layers (Chan et al., 2006; Teeters-Kennedy et al., 2007). The optomagnetic response and the negative refractive index behavior of such stacks have also been analyzed (Zhang et al., 2005; Li et al., 2006, 2007; Beruete, Campillo, et al., 2007; Beruete, Navarro-Cia, et al., 2007). Recently Mary et al. (2008) found that the electrical response of the so-called double-fishnet structure (2D hole arrays perforated on a metal-dielectric-metal stack) is dominated by the cutoff frequency of the hole waveguide whereas the resonant magnetic response is due to the excitation of gap SPPs propagating along the dielectric slab. Such results suggest further exploration of the metamaterial-like behavior of coupled aperture structures.

Finally, we anticipate much research activity from the transfer and application of the EOT phenomenon to other domains. As discussed above, the EOT process can be applied to matter waves for the realization of aperture-based optical elements which should be ideally suited for beams of atoms or other particles (Moreno et al., 2005). Introducing such elements in a cold atom setup will not be trivial but the potential seems well worth it. Giant transmissions are also observed for perforated films involving surface phonon polaritons (Korobkin et al., 2007). The acoustic equivalent of the EOT process has received much attention recently (Cai et al., 2007; Christensen et al., 2007, 2008; Lu et al., 2007; Zhou and Kriegsmann, 2007; Estrada et al., 2008; Mei et al., 2008). Beyond the interesting fundamental aspects, the applications to acoustics open numerous possibilities from sound insulation to beaming, which could rapidly find practical use.

As stated, the potential of metallic aperture structures lies in the high contrast and high local fields they provide and in the simplicity of their implementation. The capacity to further texture the metal around the apertures adds another important element which has not yet been completely evaluated. It is, therefore, expected that this will not only improve existing applications but also expand them. Overall, many developments can be expected in the years to come whether in optics or in other domains as properties of aperture structures are fully explored.

\section{ACKNOWLEDGMENTS}

F.J.G.-V. and L.M.-M. gratefully acknowledge the collaboration with many scientists during the last ten years of EOT research, in particular, Jorge Bravo-Abad, Fernando de León, Antonio Fernandez-Dominguez, Fernando López-Tejeira, Esteban Moreno, Sir John Pendry, Juan Antonio Porto, and Sergio G. Rodrigo. T.W.E. would like to thank his collaborators Eloise Devaux and Cyriaque Genet. L.K. would like to thank his collaborators Stefan Enoch, Rob Harmsen, Koen Klein Koerkamp, Jord Prangsma, Marijn Sandtke, Frans Segerink, Karen van der Molen, Niek van Hulst, and Joris van Nieuwstadt. F.J.G.-V. and L.M.-M. acknowledge the financial support from the Spanish Ministry of Education and Science while L.K. acknowledges the support of both the Smart Mix Programme of The Netherlands Ministry of Economic Affairs and The Netherlands Ministry of Education, Culture and Science and the research program of the Stichting voor Fundamenteel Onderzoek (FOM), which is financially supported by The Nederlandse Organisatie voor Wetenschappelijk Onderzoek (NWO).

\section{APPENDIX: COUPLED-MODE METHOD}

\section{Periodic systems}

In this appendix we describe the details of the coupled mode framework presented in Sec. I.B, which is used in this review to analyze the light transmission through subwavelength apertures.

We denote the direction normal to the film by $z$ and take the metal-dielectric interfaces to be located at $z$ $=0$ and $z=h$. We assume a rectangular supercell, with lattice parameters $L_{x}$ and $L_{y}$, along the $x$ and $y$ axes, respectively (see Fig. 2). This supercell may be real (if we are considering an infinitely periodic system) or artificial (if the system is finite). In this latter case, the limit $L_{x}, L_{y} \rightarrow \infty$ must be taken.

First, we analyze the expansion of the EM fields in the three different regions (reflection, perforated metal, and transmission) defining the structure. For an incident plane wave with parallel wave vector $\vec{k}_{0}$ and polarization $\sigma_{0}$, the parallel components of the EM fields at $z=0^{-}$(at 
the metal interface in which radiation is impinging on) can be written in terms of the reflection coefficients $r_{\vec{k} \sigma}$ as

$$
\begin{aligned}
& \left|\vec{E}\left(z=0^{-}\right)\right\rangle=\left|\vec{k}_{0} \sigma_{0}\right\rangle+\sum_{\vec{k} \sigma} r_{\vec{k} \sigma}|\vec{k} \sigma\rangle, \\
& -\vec{u}_{z} \times\left|\vec{H}\left(z=0^{-}\right)\right\rangle=Y_{\vec{k}_{0} \sigma_{0}}\left|\vec{k}_{0} \sigma_{0}\right\rangle-\sum_{\vec{k} \sigma} r_{\vec{k} \sigma} Y_{\vec{k} \sigma}|\vec{k} \sigma\rangle,
\end{aligned}
$$

where we have used a Dirac notation and expressed the bivectors $\langle\vec{r} \mid \vec{E}\rangle=\vec{E}(\vec{r})=\left(E_{x}(\vec{r}), E_{y}(\vec{r})\right)^{T}$ and $\langle\vec{r} \mid \vec{H}\rangle=\vec{H}(\vec{r})$ $=\left(H_{x}, H_{y}\right)^{T}$ (T standing for transposition) in terms of the EM plane wave eigenmodes, $|\vec{k} \sigma\rangle$. Note that $\vec{r}$ refers to the parallel components $(x$ and $y$ ) of the spatial vector and that the $z$ components of the EM fields can be found using the Maxwell equations and the direction of propagation of the corresponding plane wave. The expressions for these plane waves in real space are

$$
\begin{aligned}
& \langle\vec{r} \mid \vec{k} p\rangle=\left(k_{x}, k_{y}\right)^{T} \exp (i \vec{k} \cdot \vec{r}) / \sqrt{L_{x} L_{y}|k|^{2}}, \\
& \langle\vec{r} \mid \vec{k} s\rangle=\left(-k_{y}, k_{x}\right)^{T} \exp (i \vec{k} \cdot \vec{r}) / \sqrt{L_{x} L_{y}|k|^{2}} .
\end{aligned}
$$

These modes are orthonormal when integrated over a unit cell, i.e., $\left\langle\vec{k} \sigma \mid \vec{k}^{\prime} \sigma^{\prime}\right\rangle=\delta_{\vec{k}, \vec{k}^{\prime}} \delta_{\sigma, \sigma^{\prime}}$, where $\delta$ is the Kronecker delta. The electric and magnetic fields in Eqs. (A1) are related through the admittances $Y_{\vec{k} s}=k_{z} / k_{\omega}$ and $Y_{\vec{k} p}=\epsilon_{1} k_{\omega} / k_{z}$ (for $s$ and $p$ polarization, respectively), where $\epsilon_{1}$ is the dielectric constant of region I, $k_{\omega}=\omega / c(\omega$ is the frequency and $c$ is the speed of light), and $|k|^{2}$ $+k_{z}^{2}=k_{\omega}^{2}$. Note that, according to Bloch's theorem, $\vec{k}$ $=\vec{k}_{0}+\vec{k}_{R}, \vec{k}_{R}$ being a (supercell) reciprocal lattice vector. In a similar way, in the region of transmission, the EM fields at $z=h^{+}$can be expressed as a function of the transmission amplitudes $t_{\vec{k} \sigma}$ as

$$
\begin{aligned}
& \left|\vec{E}\left(z=h^{+}\right)\right\rangle=\sum_{\vec{k} \sigma} t_{\vec{k} \sigma}|\vec{k} \sigma\rangle, \\
& -\vec{u}_{z} \times\left|\vec{H}\left(z=h^{+}\right)\right\rangle=\sum_{\vec{k} \sigma} t_{\vec{k} \sigma} Y_{\vec{k} \sigma}^{\prime}|\vec{k} \sigma\rangle .
\end{aligned}
$$

In these last equations, the expression for the admittance of the corresponding plane wave in region III, $Y_{\vec{k} \sigma}^{\prime}$, is the same as in region I with $\epsilon_{1}$ replaced by $\epsilon_{3}$.

For the modal expansion of the EM fields within the indentations, we use a compact notation by denoting with object $\alpha$ to any waveguide EM mode considered in the expansion. Therefore, an object is characterized by the indentation it belongs to, by its polarization (TM or TE modes), and by the indices related to its mode spatial dependence. All that needs be known are the electric field bivectors $\langle\vec{r} \mid \alpha\rangle$ and the propagation constants $q_{z \alpha}$ associated with the object. For indentations with simple cross sections as slits or rectangular and circular holes, the required expressions for $|\alpha\rangle$ and $q_{z \alpha}$ can be found analytically. As in the case of the plane waves, the magnetic field can be related to the electric field bivector as $-\vec{u}_{z} \times|\vec{H}\rangle_{\alpha}= \pm Y_{\alpha}|\alpha\rangle$, with the admittance $Y_{\alpha}=q_{z \alpha} / k_{\omega}$ for
TE modes, while for TM modes $Y_{\alpha}=k_{\omega} / q_{z \alpha}$. Then, the electric and magnetic fields inside the indentations can be written in terms of the expansion coefficients $\left\{A_{\alpha}, B_{\alpha}\right\}$ as

$$
\begin{aligned}
& |\vec{E}(z)\rangle=\sum_{\alpha}|\alpha\rangle\left[A_{\alpha} e^{i q_{z \alpha} z}+B_{\alpha} e^{-i q_{z \alpha} z}\right], \\
& -\overrightarrow{u_{z}} \times|\vec{H}(z)\rangle=\sum_{\alpha}|\alpha\rangle Y_{\alpha}\left[A_{\alpha} e^{i q_{z \alpha} z}-B_{\alpha} e^{-i q_{z \alpha} z}\right] .
\end{aligned}
$$

The way to extract the complete set of unknowns $\left\{A_{\alpha}, B_{\alpha}, t_{\vec{k} \sigma}, r_{\vec{k} \sigma}\right\}$ is by matching the EM fields appropriately at the two horizontal interfaces $(z=0$ and $z=h)$. Within the perfect electrical conductor approximation, the parallel components of the electric field must be continuous over the whole surface whereas the magnetic field counterparts are continuous only over the apertures. As we cannot consider the infinite number of modes in the structure [i.e., the sums in Eqs. (A1), (A3), and (A4) must be truncated], these matching relations cannot be fulfilled at every point, so the matching procedure should not be done in real space. Instead, in the truncated Hilbert space, the correct procedure is to project each matching equation onto the set of modes which spans the spatial region over which the equation is defined. Hence, continuity of the $E$-field components should be expanded onto plane waves $(\langle\vec{k} \sigma|)$ and the ones related to the $H$ field should be projected onto waveguide modes $\langle\alpha|$. After this matching procedure, we end up with a system of linear equations for the expansion coefficients, $\left\{A_{\alpha}, B_{\alpha}\right\}$. It is convenient to define the related quantities $E_{\alpha} \equiv A_{\alpha}+B_{\alpha}$ and $E_{\alpha}^{\prime} \equiv-\left(A_{\alpha} e^{i q_{z \alpha} h}\right.$ $\left.+B_{\alpha} e^{-i q_{z \alpha} h}\right)$, which are the modal amplitudes of the $E$ field at the input and output interfaces of the indentations, respectively. The set $\left\{E_{\alpha}, E_{\alpha}^{\prime}\right\}$ satisfies

$$
\begin{aligned}
& \left(G_{\alpha \alpha}-\Sigma_{\alpha}\right) E_{\alpha}+\sum_{\beta \neq \alpha} G_{\alpha \beta} E_{\beta}-G_{\alpha}^{V} E_{\alpha}^{\prime}=I_{\alpha}, \\
& \left(G_{\gamma \gamma}^{\prime}-\Sigma_{\gamma}\right) E_{\gamma}^{\prime}+\sum_{\nu \neq \gamma} G_{\gamma \nu}^{\prime} E_{\nu}^{\prime}-G_{\gamma}^{V} E_{\gamma}=0 .
\end{aligned}
$$

This is the basic set of linear equations [Eqs. (1)] used throughout this review article to describe the transmission properties of different structures containing subwavelength apertures.

The different terms appearing in these "tightbinding"-like equations have the following mathematical expressions. The illumination term $I_{\alpha}$ is proportional to the overlap integral between object $\alpha$ and the incident plane wave: $I_{\alpha}=2 i Y_{\vec{k}_{0} \sigma_{0}}\left\langle\vec{k}_{0} \sigma_{0} \mid \alpha\right\rangle . \Sigma_{\alpha}$ is related to the bouncing back and forth of the EM fields inside object $\alpha$ and for an aperture its expression is $\Sigma_{\alpha}=Y_{\alpha} / \tan \left(q_{z \alpha} h\right)$. In the case in which the indentation does not completely trespass the metal (a dimple in two dimensions or a groove in one dimension), the expression for $\Sigma_{\alpha}$ is the same but $h$ must be replaced by the depth of the indentation. The coupling between the two sides of the aperture is accounted for $G_{\alpha}^{V}=Y_{\alpha} / \sin \left(q_{z \alpha} h\right)$. In the case of a 
waveguide mode $\alpha$ associated with a dimple or a groove, $G_{\alpha}^{V}=0$ leading to $E_{\alpha}^{\prime}=0$. The term $G_{\alpha \beta}$ controls the EM coupling between objects $\alpha$ and $\beta$ :

$$
G_{\alpha \beta}=i \sum_{\vec{k} \sigma} Y_{\vec{k} \sigma}\langle\alpha \mid \vec{k} \sigma\rangle\langle\vec{k} \sigma \mid \beta\rangle,
$$

where the overlap $\langle\alpha \mid \vec{k} \sigma\rangle$ involves the evaluation of an integral in real space:

$$
\langle\alpha \mid \vec{k} \sigma\rangle=\int d \vec{r}\langle\alpha \mid \vec{r}\rangle\langle\vec{r} \mid \vec{k} \sigma\rangle .
$$

If the system is periodic, $G_{\alpha \beta}$ can be easily calculated through the previous discrete sum [Eq. (A6)] by including enough diffraction waves. Within the coupled-mode formalism, the finite dielectric function of a real metal can be incorporated in an approximated way by means of the surface impedance boundary conditions (LópezTejeira et al., 2005; de Leon-Perez et al., 2008). The important point of this approach is that the system of linear equations (A5) still holds; only the different magnitudes appearing on it differ from their PEC values:

$$
\begin{aligned}
& I_{\alpha}=\frac{2 i Y_{\vec{k}_{0} \sigma_{0}}\left\langle\vec{k}_{0} \sigma_{0} \mid \alpha\right\rangle}{1+Z_{S} Y_{\vec{k}_{0} \sigma_{0}}} \\
& \Sigma_{\alpha}=i Y_{\alpha} \frac{e^{i q_{z \alpha} h}\left(1+Z_{S} Y_{\alpha}\right)+e^{-i q_{z \alpha} h}\left(1-Z_{S} Y_{\alpha}\right)}{e^{i q_{z \alpha} h}\left(1+Z_{S} Y_{\alpha}\right)^{2}-e^{-i q_{z \alpha} h}\left(1-Z_{S} Y_{\alpha}\right)^{2}}, \\
& G_{\alpha}^{V}=\frac{2 i Y_{\alpha}}{e^{i q_{z \alpha} h}\left(1+Z_{S} Y_{\alpha}\right)^{2}-e^{-i q_{z \alpha} h}\left(1-Z_{S} Y_{\alpha}\right)^{2}},
\end{aligned}
$$

where $Z_{S}=1 / \sqrt{\epsilon_{M}(\omega)}$ is the surface impedance of the metal. The expression for $\Sigma_{\alpha}$ for a dimple or a groove of depth $w$ is

$$
\Sigma_{\alpha}=i Y_{\alpha} \frac{e^{i q_{z \alpha^{w}}}\left(1-Z_{S} Y_{\alpha}\right)+e^{-i q_{z \alpha^{w}}}\left(1+Z_{S} Y_{\alpha}\right)}{\left(1-Z_{S}^{2} Y_{\alpha}^{2}\right)\left(e^{i q_{z \alpha^{w}}}-e^{-i q_{z \alpha^{w}}}\right)} .
$$

The coupling term $G_{\alpha \beta}$ also depends on $Z_{S}$ :

$$
G_{\alpha \beta}=i \sum_{\vec{k} \sigma} \frac{Y_{\vec{k} \sigma}}{1+Z_{S} Y_{\vec{k} \sigma}}\langle\alpha \mid \vec{k} \sigma\rangle\langle\vec{k} \sigma \mid \beta\rangle .
$$

\section{Multiple scattering}

We now describe how to evaluate the different twomedia scattering coefficients as shown in Fig. 3 using the coupled-mode formalism. By applying a matching procedure equivalent to that used for obtaining the set of equations (A5), we can relate the different two-media scattering coefficients with those magnitudes appearing in the general set of equations (A5). For example, for the scattering process shown in Fig. 3(a), we obtain the following set of equations for the different $\tau_{\alpha}^{12}$ :

$$
\left(G_{\alpha \alpha}+i Y_{\alpha}\right) \tau_{\alpha}^{12}+\sum_{\beta \neq \alpha} G_{\alpha \beta} \tau_{\beta}^{12}=I_{\alpha}
$$

From them, it is then possible to extract the reflection coefficients associated with the interface I-II, $\rho_{\vec{k} \sigma}^{12}$ :

$$
\rho_{\vec{k} \sigma}^{12}=-\delta_{\vec{k} \vec{k}_{0}} \delta_{\sigma \sigma_{0}}+\sum_{\alpha}\langle\vec{k} \sigma \mid \alpha\rangle \tau_{\alpha}^{12}
$$

The second two-media scattering problem is schematically shown in Fig. 3(b). The matching of the parallel components of the EM fields leads to a system of linear equations for $\rho_{\alpha \beta}^{23}$ :

$$
\left(G_{\gamma \gamma}^{\prime}+i Y_{\gamma}\right) \rho_{\alpha \gamma}^{23}+\sum_{\beta \neq \gamma} G_{\gamma \beta}^{\prime} \rho_{\alpha \beta}^{23}=i Y_{\gamma} \delta_{\gamma \alpha}-G_{\gamma \alpha}^{\prime} .
$$

The transmission coefficients in medium III can be extracted from the knowledge of $\left\{\rho_{\alpha \beta}^{23}\right\}$ by means of

$$
\tau_{\alpha, \vec{k} \sigma}^{23}=\langle\vec{k} \sigma \mid \alpha\rangle+\sum_{\beta} \rho_{\alpha \beta}^{23}\langle\vec{k} \sigma \mid \beta\rangle .
$$

The procedure for extracting the two-media coefficients associated with the II-I scattering problem is completely equivalent to that of II-III previously discussed. Once the two-media scattering coefficients are known it is possible to recover the three-media coefficients [see, for instance, Eq. (6)].

\section{Finite systems}

Up to this point, we have described the theoretical framework able to deal with infinitely periodic structures (2D arrays of holes or 1D arrays of slits). The only difference between infinite and finite structures resides in the calculation of $G_{\alpha \beta}$ [the rest of the magnitudes appearing in Eqs. (A5) are the same]. When treating a finite set of indentations, the limit $L_{x}, L_{y} \rightarrow \infty$ must be taken. In this way, $G_{\alpha \beta}$ is calculated by means of an integral over diffraction modes:

$$
G_{\alpha \beta}=i \frac{L_{x} L_{y}}{(2 \pi)^{2}} \sum_{\sigma} \int d \vec{k} \frac{Y_{\vec{k} \sigma}}{1+Z_{S} Y_{\vec{k} \sigma}}\langle\alpha \mid \vec{k} \sigma\rangle\langle\vec{k} \sigma \mid \beta\rangle,
$$

where the expression for a PEC can be obtained from Eq. (A14) by simply setting $Z_{S}=0$. In Eq. (A14), the integration is over all possible $\vec{k}$ (in-plane components of the wave vector) and the factor $L_{x} L_{y}$ cancels out with that coming from the normalization of the EM plane waves. Alternatively, $G_{\alpha \beta}$ can be calculated as a double integral in real space using

$$
G_{\alpha \beta} \equiv\langle\alpha|\hat{G}| \beta\rangle=\iint d \vec{r} d \vec{r}^{\prime}\langle\alpha \mid \vec{r}\rangle \hat{G}\left(\vec{r}, \vec{r}^{\prime}\right)\left\langle\vec{r}^{\prime} \mid \beta\right\rangle,
$$

where $\hat{G}\left(\vec{r}, \vec{r}^{\prime}\right)$ is related to the Green's dyadic tensor in a metal-dielectric interface whose formal expression can be extracted by comparing Eq. (A15) with Eq. (A14): 


$$
\hat{G}\left(\vec{r}, \vec{r}^{\prime}\right)=\frac{i L_{x} L_{y}}{(2 \pi)^{2}} \int d \vec{k} \frac{Y_{\vec{k} \sigma}}{1+Z_{S} Y_{\vec{k} \sigma}}\langle\vec{r} \mid \vec{k} \sigma\rangle\left\langle\vec{k} \sigma \mid \vec{r}^{\prime}\right\rangle .
$$

Thus, the dyadic $\hat{G}\left(\vec{r}, \vec{r}^{\prime}\right)$ controls the EM coupling between apertures. Note that the calculation of $G_{\alpha \beta}$ from Eq. (A15) for waveguide modes in the same indentation suffers from problems associated with the divergence of the dyadic $\hat{G}\left(\vec{r}, \vec{r}^{\prime}\right)$ at $\left|\vec{r}-\vec{r}^{\prime}\right| \rightarrow 0$. For this case, $G_{\alpha \beta}$ must be evaluated as an integral over diffraction modes [Eq. (A14)].

In order to illustrate the spatial dependence of the dyadic $\hat{G}\left(\vec{r}, \vec{r}^{\prime}\right)$, we concentrate on the case of $1 \mathrm{D}$ geometries illuminated by $p$-polarized light (and with zero component of the incident wave vector along the axis of symmetry of the structure). The 1D Green's function for $p$-polarized light within the SIBC approach $G_{p}\left(x, x^{\prime}\right)$ can be written as

$$
G_{p}\left(x, x^{\prime}\right)=\frac{i}{2 \pi} \int_{-\infty}^{\infty} d k \frac{k_{w}}{\sqrt{k_{w}^{2}-k^{2}}+Z_{S}} e^{i k\left(x-x^{\prime}\right)} .
$$

It is worth noting that the corresponding expression for a PEC $\left[Z_{S}=0\right.$ in Eq. (A17)] can be analytically evaluated and is related to the zero-order Hankel function of the first kind $H_{0}^{(1)}$ :

$$
G_{p}^{\mathrm{PEC}}\left(x, x^{\prime}\right)=\frac{i \pi}{\lambda} H_{0}^{(1)}\left(k_{w}\left|x-x^{\prime}\right|\right) .
$$

When considering a single slit perforated in a PEC film (Sec. II.A), we need to evaluate the coupling term $G=G_{00}$ for the fundamental TEM waveguide mode. As commented above, this term can be evaluated either by means of a double integral in real space or via an integral over vacuum plane waves,

$$
\begin{aligned}
G_{00} & =\frac{i \pi}{a \lambda} \int_{-a / 2}^{a / 2} d x \int_{-a / 2}^{a / 2} d x^{\prime} H_{0}^{(1)}\left(k_{\omega}\left(x-x^{\prime}\right)\right) \\
& =\frac{i a k_{w}}{2 \pi} \int_{-\infty}^{\infty} d k \frac{\operatorname{sinc}^{2}(k a / 2)}{\sqrt{k_{w}^{2}-k^{2}}},
\end{aligned}
$$

where the function $\operatorname{sinc}(x) \equiv \sin / x$.

It is interesting to compare the behavior of the $1 \mathrm{D}$ Green's dyadic function for $p$ polarization for a real metal [Eq. (A17)] with that of a PEC [Eq. (A18)]. In comparison with the PEC case, $G_{p}\left(x, x^{\prime}\right)$ must be calculated numerically. For a good metal (silver or gold), however, this integral is dominated by the single pole of the integrand due to the presence of SPPs (recall that, within the SIBC approach, the dispersion relation of SPPs reads $k_{z}=-Z_{S} k_{\omega}$, i.e., $k_{\mathrm{SPP}}=k_{\omega} \sqrt{1-Z_{S}^{2}}$ ). Isolating the contribution from the pole gives an approximation to $G_{p}\left(x, x^{\prime}\right)$ :

$$
G_{p}^{\mathrm{SPP}}\left(x, x^{\prime}\right)=-\frac{Z_{S}}{\sqrt{1-Z_{S}^{2}}} k_{\omega} e^{i k_{\mathrm{SPP}}\left|x-x^{\prime}\right|} .
$$

The behavior of the 1D Green's dyadic function is shown in Fig. 58, which presents $G_{p}\left(x, x^{\prime}\right)$ for silver at

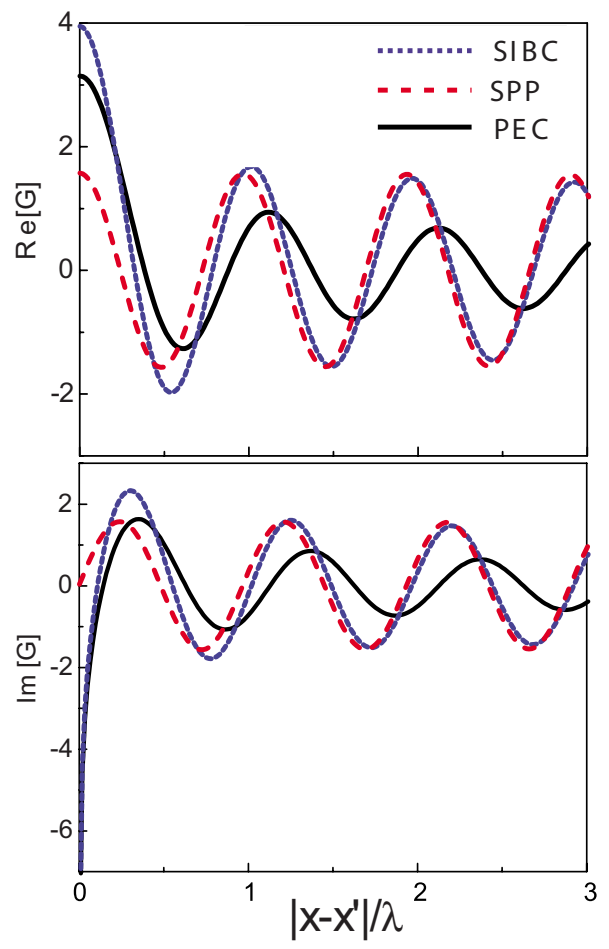

FIG. 58. (Color online) 1D Green's dyadic function (real and imaginary parts) for a Ag-vacuum interface and $p$-polarized light of $\lambda=600 \mathrm{~nm}$. The dashed curves correspond to the SIBC approach [Eq. (A17)], whereas the black curves are for a PEC [Eq. (A18)]. The dotted curves display the SPP contribution to $G$, as given by Eq. (A20).

$\lambda=600 \mathrm{~nm}$. Together with the exact numerical evaluation of Eq. (A17), we also present the calculation for a PEC [Eq. (A18)] and the "asymptotic" expression $G_{p}^{\mathrm{SPP}}$, as obtained from Eq. (A20). As Fig. 58 shows, the exact $G_{p}\left(x, x^{\prime}\right)$ resembles the PEC result at very small distances $\left|x-x^{\prime}\right| \ll \lambda$ and is well approximated by $G_{p}^{\mathrm{SPP}}$ for $\left|x-x^{\prime}\right| \geqslant 2 \lambda$.

The 1D Green's function for $s$-polarized light is different than the $p$-polarized case due to the different admittances of $s$-polarized plane waves. Within the PEC approximation, this 1D Green's function reads

$$
G_{s}\left(x, x^{\prime}\right)=\frac{i}{2 \pi} \int_{-\infty}^{\infty} d k \frac{\sqrt{k_{\omega}^{2}-k^{2}}}{k_{\omega}} e^{i k\left(x-x^{\prime}\right)} .
$$

The Green's dyadic tensor for the 2D case (holes) is more complex and further details on how to calculate it can be found in Bravo-Abad, Garcia-Vidal, and MartinMoreno (2004). For example, the expression for the selfillumination of the fundamental TE mode via vacuum modes $G$ for the case of a single rectangular hole is

$$
\begin{aligned}
G= & \frac{i a_{x} a_{y}}{8 \pi^{2} k_{\omega}} \int_{-\infty}^{+\infty} \int_{-\infty}^{+\infty} d k_{x} d k_{y} \frac{k_{\omega}^{2}-k_{y}^{2}}{\sqrt{k_{\omega}^{2}-k^{2}}} \operatorname{sinc}^{2}\left(\frac{k_{x} a_{x}}{2}\right) \\
& \times\left[\operatorname{sinc}\left(\frac{k_{y} a_{y}+\pi}{2}\right)+\operatorname{sinc}\left(\frac{k_{y} a_{y}-\pi}{2}\right)\right]^{2},
\end{aligned}
$$

where $k^{2}=k_{x}^{2}+k_{y}^{2}$. 
The formulation in real space necessary to describe finite systems could also be used to compute the optical properties of an infinitely periodic system. In this case, we know that the modal coefficients must satisfy Bloch's theorem, $E_{\alpha}\left(\vec{R}_{n}\right)=E_{\alpha} e^{i \vec{k}_{0} \cdot \vec{R}_{n}}$. Then, the coupling term between Bloch's modes $\alpha$ and $\beta$ can be evaluated in real space as a sum over lattice locations,

$$
G_{\alpha \beta}=\sum_{\vec{R}_{m}} G_{\alpha \beta}^{\vec{R} n \cdot \vec{R}_{m}} e^{i \vec{k}_{0} \cdot\left(\vec{R}_{m}-\vec{R}_{n}\right),}
$$

where $G_{\alpha \beta}^{\vec{R}_{n} \cdot \vec{R}_{m}}$ is the EM coupling between waveguide mode $\alpha$ of the hole located at $\vec{R}_{n}$ and waveguide mode $\beta$ belonging to a hole placed in $\vec{R}_{m}: G_{\alpha \beta}^{\vec{R}_{n} \cdot \vec{R}_{m}}$ $=\left\langle\alpha\left(\vec{R}_{n}\right)|\hat{G}| \beta\left(\vec{R}_{m}\right)\right\rangle$. Of course, expression (A23), which involves sums of difficult Sommerfeld integrals, is numerically much more complicated than Eq. (A6). However, Eq. (A23) is valuable as it sheds light on the origin of the divergencies of $G_{\alpha \beta}$ : they originate from constructive sums of reillumination processes of light coming from other holes (Bravo-Abad, Garcia-Vidal, et al., 2004; De Abajo and Saenz, 2005).

\section{Equations in Fourier space}

The dependence of the optical transmittance through a set of holes on their structure factor $[S(\vec{q})$ $\left.=\Sigma_{\vec{R}} \exp (-i \vec{q} \cdot \vec{R})\right]$ can be made explicit by working in Fourier space. For this, we write the Fourier components of the modal amplitudes as $E_{n}(\vec{q})$ $=\Sigma_{\vec{R}} \exp (-i \vec{q} \cdot \vec{R}) E_{n}(\vec{R})$. Note that now $E_{n}(\vec{R})$ refers to the modal amplitude in real space of mode $n$ at the hole location $\vec{R}$. By applying a Fourier transform to the set of Eqs. (A5) [further details can be found in Bravo-Abad, Fernandez-Dominguez, et al. (2007)], we obtain a set of equations that governs $\left\{E_{n}(\vec{q}), E_{n}^{\prime}(\vec{q})\right\}$ :

$$
\begin{aligned}
& {\left[\Omega_{n}(\vec{q})-\Sigma_{n}\right] E_{n}(\vec{q})-G_{n}^{V} E_{n}^{\prime}(\vec{q})=I_{n} S\left(\vec{q}-\vec{k}_{0}\right),} \\
& {\left[\Omega_{n}^{\prime}(\vec{q})-\Sigma_{n}\right] E_{n}^{\prime}(\vec{q})-G_{n}^{V} E_{n}(\vec{q})=0,}
\end{aligned}
$$

where $\vec{k}_{0}$ is the in-plane component of the incident wave vector. Note that the set of equations (A24) is just the $k$-space version of the set of linear equations (A5).

The reillumination term in Eqs. (A24), $\Omega_{n}^{(\prime)}(\vec{q}) E_{n}^{(\prime)}(\vec{q})$, is now represented by the scattering process that couples $E_{n}^{(\prime)}(\vec{q})$ to the continuum $E_{m}^{(\prime)}(\vec{k})$, the momentum difference being provided by the lattice through $S(\vec{q}-\vec{k})$ :

$$
\Omega_{n}^{(\prime)}(\vec{q}) E_{n}^{(\prime)}(\vec{q})=\sum_{m} \int d \vec{k} G_{m n ; \vec{k}} S(\vec{q}-\vec{k}) E_{m}^{(\prime)}(\vec{k}) .
$$

The amplitude of this process is governed by $G_{m n ; \vec{k}}$ :

$$
G_{m n ; \vec{k}}=\frac{i}{(2 \pi)^{2}} \sum_{\sigma} \frac{Y_{\vec{k} \sigma}}{1+Z_{S} Y_{\vec{k} \sigma}}\langle n \mid \vec{k} \sigma\rangle\langle\vec{k} \sigma \mid m\rangle .
$$

An important property is that $G_{m n ; \vec{k}}$ diverges whenever the $z$ component of the wave vector of a $p$-polarized diffraction wave coincides with that of a SPP propagating on a planar metal surface $\left(k_{z}+Z_{S} k_{\omega}=0\right)$, as can be directly seen from Eq. (A26). Examining Eq. (A25), it is clear when and where resonant peaks would emerge in the transmission spectrum: whenever a divergence in $G_{m n ; \vec{k}}$ coincides with a peak in the structure factor $S(\vec{q})$ the reillumination from all other holes characterized by $\Omega_{n} E_{n}$ is maximum.

\section{Bound modes in holey PEC surfaces and films}

The set of equations (A5) can also be used to obtain the dispersion relation of modes bound to the periodic system. Eigenmodes have strong electric fields at the surface even for arbitrarily small illuminations, i.e., they appear as solutions of the eigenvalue problem by means of the homogeneous set of equations (A5). This problem must, in general, be solved numerically. However, the structure of the dispersion relation can be obtained analytically using two approximations: (i) only the fundamental mode inside the indentation is taken into account and (ii) only $p$-polarized waves and the zero-order diffraction mode are considered in the quantity $G$ governing the coupling between indentations. The first approximation provides accurate results for subwavelength apertures and allows to work with the single mode version of Eqs. (A5) [Eqs. (3)]. The second approximation, which is equivalent to consider the system as a metamaterial represented by the average EM fields, must be corrected close to the edges of the Brillouin zone, where gaps still open due to the ignored diffraction orders. After inclusion of diffraction orders, the lower band is an EM mode fully bound to the surface, while higher bands represent leaky modes which are coupled to radiation. A second effect of approximation (ii) is that, away from the edges of the Brillouin zone, it has been found to overestimate the binding of the EM modes (De Abajo and Saenz, 2005). With these caveats in mind, these approximations predict that periodic arrays of indentations of depth $w$, closed at the bottom, bind modes in a PEC. This occurs when the condition $G(\vec{k})-\Sigma=0$ is satisfied, where $\Sigma=Y(\phi+1 / \phi) /(\phi-1 / \phi)$, with $\phi=e^{i q w}, Y$ being the admittance of the fundamental mode and $q$ its wave vector along the $z$ direction. All dependence on the parallel component of the incident wave vector $\vec{k}$ is contained in $G(\vec{k}) \equiv k_{\omega} / \sqrt{k_{\omega}^{2}-k^{2}} S(\vec{k})^{2}$, where $S(\vec{k}) \equiv\langle\vec{k} p \mid 0\rangle$ and $|0\rangle$ denotes the fundamental mode. The solution to the previous condition must, in general, be computed numerically. However, for subwavelength apertures and moderate wave vectors the dependence of the overlap $S(\vec{k})$ on the wave vector can be neglected, $S(\vec{k}) \approx S$. Then the dispersion relation of bound EM modes is

$$
k= \pm k_{\omega} \sqrt{1+S^{4} /|\Sigma|^{2}},
$$

where $k=|\vec{k}|$. The bound nature of the modes is reflected in the property $k>k_{\omega}$. Moreover, this equation predicts 
a horizontal asymptote whenever $\Sigma=0$. As the existence of this asymptote is reminiscent of that in the dispersion relation of surface plasmons, the bound modes in a holey PEC surface were termed spoof surface plasmons.

If the fundamental waveguide mode is propagating (1D array of grooves for $p$-polarized light), the condition $\Sigma=0$ occurs at $\phi=-1 / \phi$, leading to $q=(2 m+1) \pi /(2 w)$, i.e., at the occurrence of cavity modes. For evanescent fundamental TE waveguide modes (2D array of holes), $\Sigma=0$ for $q=0$, i.e., at the cutoff frequency of the waveguide mode (Pendry et al., 2004). These properties are reflected in the explicit dispersion relations for spoof surface plasmons in a 1D array of grooves of width $a$ and depth $w$,

$$
k_{x}=k_{\omega} \sqrt{1+\left(a^{2} / d^{2}\right) \tan ^{2}\left(k_{\omega} w\right)},
$$

and for a 2D square array of square dimples (holes closed at the bottom) of side $a$ and depth $w$,

$$
k=k_{\omega} \sqrt{1+\frac{k_{\omega}^{2} S^{4} \beta^{2}}{(\pi / a)^{2}-k_{\omega}^{2}}},
$$

with $S=2 \sqrt{2} a / \pi d$ and $\beta=\left(e^{|q| w}-e^{-|q| w}\right) /\left(e^{|q| w}+e^{-|q| w}\right)$.

Similarly, it is possible to obtain the modes bound to a PEC film perforated with holes that fully trespass the film of thickness $h$. In a symmetric environment, where dielectric constants in media I and III are equal, the solutions to the homogeneous system of equations (3) satisfy either $E=-E^{\prime}$ or $E=E^{\prime}$. Recalling the change of sign in the definition of $E^{\prime}$, the former corresponds to a mode with parallel components of the electric field symmetric with respect to the mirror plane at $z=h / 2$. After some straightforward algebra, the dispersion relation of the symmetric EM mode bound to a metal film perforated with a 2D square array of subwavelength square holes is

$$
k=k_{\omega} \sqrt{1+\frac{k_{\omega}^{2} S^{4} \gamma^{2}}{(\pi / a)^{2}-k_{\omega}^{2}}}
$$

while the antisymmetric mode satisfies

$$
k=k_{\omega} \sqrt{1+\frac{k_{\omega}^{2} S^{4} \gamma^{-2}}{(\pi / a)^{2}-k_{\omega}^{2}}},
$$

where $\gamma=\left(e^{|q| h / 2}+e^{-|q| h / 2}\right) /\left(e^{|q| h / 2}-e^{-|q| h / 2}\right)$. This quantity satisfies $\gamma \geqslant 1$ and increases with decreasing film thickness. As $h$ decreases the dispersion relation of the antisymmetric mode moves toward the light line $\left(k=k_{\omega}\right)$, while the one corresponding to the symmetric mode moves away from it. This is in agreement with the intuitive view that the "molecular" EM modes in a film originate from the coupling of the "atomic" modes in the isolated interfaces via the evanescent waveguide modes. The frequency splitting between the bound EM modes depends strongly on the strength of the coupling, and therefore on $h$.

In the metamaterial limit (wavelength much larger than the typical dimensions of the structure), the geometrically induced surface EM modes can be interpreted as those supported by an effective metamaterial charac- terized by an electric permittivity $\left(\epsilon_{\text {eff }}\right)$ and a magnetic permeability $\left(\mu_{\mathrm{eff}}\right)$. These two effective parameters can be extracted by working with the condition for bound modes $[G(\vec{k})-\Sigma=0]$ in the case of a semi-infinite structure $(\Sigma=Y)$. By assuming $S(\vec{k}) \approx S$, the above condition can be rewritten as $k_{\omega} / \sqrt{k_{\omega}^{2}-k^{2}}=Y / S^{2}$. In a semi-infinite homogeneous medium, the condition for surface mode excitation is $k_{\omega} / \sqrt{k_{\omega}^{2}-k^{2}}=\sqrt{\epsilon_{\text {eff }} / \mu_{\text {eff. }}}$. Therefore, a perforated semi-infinite PEC can be considered as a homogeneous metamaterial satisfying $\sqrt{\epsilon_{\text {eff }} / \mu_{\text {eff }}}=Y / S^{2}$. The other relation that involves both $\epsilon_{\text {eff }}$ and $\mu_{\text {eff }}$ is associated with the $z$ component of the wave vector, $q$ $=k_{\omega} \sqrt{\epsilon_{\text {eff }} \mu_{\text {eff }}}$. By working with these last equations, two general expressions for $\epsilon_{\text {eff }}$ and $\mu_{\text {eff }}$ can be found:

$$
\epsilon_{\mathrm{eff}}=Y q / k_{\omega} S^{2}, \quad \mu_{\mathrm{eff}}=q S^{2} / Y k_{\omega} .
$$

For the 2D case (periodic array of holes or dimples), $Y=q / k_{w}$ with $q=\sqrt{\epsilon_{\text {hole }} k_{\omega}^{2}-\pi^{2} / a^{2}}$ ( $\epsilon_{\text {hole }}$ being the dielectric constant of the medium filling the holes), yielding $\mu_{\text {eff }}=S^{2}$ and $\epsilon_{\text {eff }}$ given by

$$
\epsilon_{\text {eff }}=\left(\epsilon_{\text {hole }} / S^{2}\right)\left(1-\pi^{2} / a^{2} k_{\omega}^{2} \epsilon_{\text {hole }}\right) .
$$

This result, which coincides with Eq. (27), was first derived by Pendry et al. (2004) and suggests that a holey PEC film behaves as a Drude-like metal with a plasma frequency that is just the cutoff frequency of the hole waveguide.

\section{REFERENCES}

Adam, A. J. L., J. M. Brok, M. A. Seo, K. J. Ahn, D. S. Kim, J. H. Kang, Q. H. Park, M. Nagel, and P. C. M. Planken, 2008, Opt. Express 16, 7407.

Agrawal, A., H. Cao, and A. Nahata, 2005, Opt. Express 13, 3535 .

Agrawal, A., and A. Nahata, 2006, Opt. Express 14, 1973.

Airola, M., Y. Liu, and S. Blair, 2005, J. Opt. A, Pure Appl. Opt. 7, S118.

Akarca-Biyikli, S. S., I. Bulu, and E. Ozbay, 2004, Appl. Phys. Lett. 85, 1098.

Alkaisi, M. M., R. B. Blaikie, S. J. McNab, R. Cheung, and D. R. S. Cumming, 1999, Appl. Phys. Lett. 75, 3560.

Alloschery, O., R. Mathevet, J. Weiner, and H. J. Lezec, 2006, Opt. Express 14, 12568.

Altewischer, E., C. Genet, M. P. van Exter, J. P. Woerdman, P. F. A. Alkemade, A. van Zuuk, and E. W. J. M. van der Drift, 2005, Opt. Lett. 30, 90.

Altewischer, E., X. Ma, M. P. van Exter, and J. P. Woerdman, 2005, Opt. Lett. 30, 2436.

Altewischer, E., Y. C. Oei, M. P. van Exter, and J. P. Woerdman, 2005, Phys. Rev. A 72, 013817.

Altewischer, E., M. P. van Exter, and J. P. Woerdman, 2002, Nature (London) 418, 304.

Altewischer, E., M. P. van Exter, and J. P. Woerdman, 2003, J. Opt. Soc. Am. B 20, 1927.

Altewischer, E., M. P. van Exter, and J. P. Woerdman, 2005, J. Opt. Soc. Am. B 22, 1731.

Altewischer, E., M. P. van Exter, and J. P. Woerdman, 2006, New J. Phys. 8, 57.

Astilean, S., P. Lalanne, and M. Palamaru, 2000, Opt. Com- 
mun. 175, 265.

Auckland, D. T., and R. F. Harrington, 1978, IEEE Trans. Microwave Theory Tech. MTT-26, 499.

Azad, A. K., Y. G. Zhao, W. L. Zhang, and M. X. He, 2006, Opt. Lett. 31, 2637.

Baida, F. I., 2007, Appl. Phys. B: Lasers Opt. 89, 145.

Baida, F. I., and D. Van Labeke, 2002, Opt. Commun. 209, 17.

Baida, F. I., and D. Van Labeke, 2003, Phys. Rev. B 67, 155314.

Baida, F. I., D. Van Labeke, A. Moreau, G. Granet, and A. Belkhir, 2004, Appl. Phys. B: Lasers Opt. 79, 1.

Barbara, A., P. Quemerais, E. Bustarret, and T. Lopez-Rios, 2002, Phys. Rev. B 66, 161403.

Barnes, W. L., 1998, J. Mod. Opt. 45, 661.

Barnes, W. L., A. Dereux, and T. W. Ebbesen, 2003, Nature (London) 424, 824.

Barnes, W. L., W. A. Murray, J. Dintinger, E. Devaux, and T. W. Ebbesen, 2004, Phys. Rev. Lett. 92, 107401.

Batulla, A., Y. L. Lu, R. J. Knize, K. Reinhardt, and S. C. Chen, 2007, Opt. Express 15, 14629.

Baudrion, A.-K., F. de Leon-Perez, O. Mahboub, A. Hohenau, H. Ditlbacher, F. J. Garcia-Vidal, J. Dintinger, T. W. Ebbesen, L. Martin-Moreno, and J. R. Krenn, 2008, Opt. Express 16, 3420.

Bell, P. M., J. B. Pendry, L. M. Moreno, and A. J. Ward, 1995, Comput. Phys. Commun. 85, 306.

Beruete, M., I. Campillo, J. S. Dolado, J. E. Rodriguez-Seco, E. Perea, and M. Sorolla, 2004, IEEE Antennas Wireless Propag. Lett. 3, 328.

Beruete, M., I. Campillo, J. E. Rodriguez-Seco, E. Perea, M. Navarro-Cia, I. J. Nunez-Manrique, and M. Sorolla, 2007, IEEE Microw. Wirel. Compon. Lett. 17, 831.

Beruete, M., M. Navarro-Cia, M. Sorolla, and I. Campillo, 2007, Opt. Express 15, 8215.

Beruete, M., M. Sorolla, I. Campillo, J. S. Dolado, L. MartinMoreno, J. Bravo-Abad, and F. J. Garcia-Vidal, 2004, Opt. Lett. 29, 2500.

Bethe, H., 1944, Phys. Rev. 66, 163.

Betzig, E., and R. J. Chichester, 1993, Science 262, 1422.

Betzig, E., and J. K. Trautman, 1992, Science 257, 189.

Bliek, P. J., L. C. Botten, R. Deleuil, R. C. McPhedran, and D. Maystre, 1980, IEEE Trans. Microwave Theory Tech. MTT28, 1119.

Blom, H., L. Kastrup, and C. Eggeling, 2006, Curr. Pharm. Biotechnol. 7, 51.

Botten, L. C., R. C. McPhedran, and J. M. Lamarre, 1985, Int. J. Infrared Millim. Waves 6, 511.

Bouwkamp, C. J., 1950, Philips Res. Rep. 5, 401.

Bravo-Abad, J., A. Degiron, F. Przybilla, C. Genet, F. J. Garcia-Vidal, L. Martin-Moreno, and T. W. Ebbesen, 2006, Nat. Phys. 2, 120.

Bravo-Abad, J., A. I. Fernandez-Dominguez, F. J. GarciaVidal, and L. Martin-Moreno, 2007, Phys. Rev. Lett. 99, 203905.

Bravo-Abad, J., F. J. Garcia-Vidal, and L. Martin-Moreno, 2003, Photonics Nanostruct. Fundam. Appl. 1, 55.

Bravo-Abad, J., F. J. Garcia-Vidal, and L. Martin-Moreno, 2004, Phys. Rev. Lett. 93, 227401.

Bravo-Abad, J., L. Martin-Moreno, and F. J. Garcia-Vidal, 2004, Phys. Rev. E 69, 026601.

Bravo-Abad, J., L. Martin-Moreno, F. J. Garcia-Vidal, E. Hendry, and J. Gomez Rivas, 2007, Phys. Rev. B 76, 241102(R).

Brolo, A. G., E. Arctander, R. Grodon, and K. L. Kavanagh, 2004, Nano Lett. 4, 2015.
Brolo, A. G., R. Gordon, B. Leathem, and K. L. Kavanagh, 2004, Langmuir 20, 4813.

Brolo, A. G., S. C. Kwok, M. D. Cooper, M. G. Moffitt, C. W. Wang, R. Gordon, J. Riordon, and K. L. Kavanagh, 2006, J. Phys. Chem. B 110, 8307.

Brolo, A. G., S. C. Kwok, M. G. Moffit, R. Gordon, J. Riordon, and K. L. Kavanagh, 2005, J. Am. Chem. Soc. 127, 14936.

Bulu, I., H. Caglayan, and E. Ozbay, 2005, Opt. Lett. 30, 3078. Caglayan, H., I. Bulu, and E. Ozbay, 2006a, IEEE Microwave Opt. Technol. Lett. 48, 2491.

Caglayan, H., I. Bulu, and E. Ozbay, 2006b, J. Opt. Soc. Am. B 23, 419.

Cai, F., F. Liu, Z. He, and Z. Liu, 2007, Appl. Phys. Lett. 91, 203515.

Cao, H., A. Agrawal, and A. Nahata, 2005, Opt. Express 13, 763.

Cao, H., and A. Nahata, 2004a, Opt. Express 12, 1004.

Cao, H., and A. Nahata, 2004b, Opt. Express 12, 3664.

Cao, Q., and P. Lalanne, 2002, Phys. Rev. Lett. 88, 057403.

Catrysse, P. B., H. Shin, and S. Fan, 2005, J. Vac. Sci. Technol. B 23, 2675.

Catrysse, P. B., G. Veronis, H. Shin, J.-T. Shen, and S. Fan, 2006, Appl. Phys. Lett. 88, 031101.

Chan, H. B., Z. Marcet, K. Woo, D. B. Tanner, D. W. Carr, J. E. Bower, R. A. Cirelli, E. Ferry, F. Klemens, J. Miner, C. S. Pai, and J. A. Taylor, 2006, Opt. Lett. 31, 516.

Chang, C. K., D. Z. Lin, C. S. Yeh, C. K. Lee, Y. C. Chang, M. W. Lin, J. T. Yeh, and J. M. Liu, 2006, Opt. Lett. 31, 2341.

Chang, S.-H., S. K. Gray, and G. C. Schatz, 2005, Opt. Express 13, 3150.

Chang, W. L., P.-H. Tsao, and P.-K. Wei, 2007, Opt. Lett. 32, 71. Chang, Y.-T., T.-H. Chuang, M.-W. Tsai, M.-J. Lai, L.-C. Chen, and S.-C. Lee, 2007, J. Appl. Phys. 101, 054305.

Chen, C.-C., 1970, IEEE Trans. Microwave Theory Tech. MTT-18, 627.

Chen, C.-C., 1971, IEEE Trans. Microwave Theory Tech. MTT-19, 475.

Chen, C.-C., 1973, IEEE Trans. Microwave Theory Tech. MTT-21, 1.

Chen, C.-Y., M.-W. Tsai, T.-H. Chuang, Y.-T. Chang, and S.-C. Lee, 2007, Appl. Phys. Lett. 91, 063108.

Chen, D. Z. A., R. Hamam, M. Soljacic, and J. D. Joannopoulos, 2007, Appl. Phys. Lett. 90, 181921.

Chen, L., J. T. Robinson, and M. Lipson, 2006, Opt. Express 14, 12629.

Chen, Z., I. R. Hooper, and J. R. Sambles, 2008, Phys. Rev. B 77, 161405.

Chowdhury, M. H., J. M. Catchmark, and J. R. Lakowicza, 2007, Appl. Phys. Lett. 91, 103118.

Christensen, J., A. I. Fernandez-Dominguez, F. de Leon-Perez, L. Martin-Moreno, and F. J. Garcia-Vidal, 2007, Nat. Phys. 3, 851.

Christensen, J., L. Martin-Moreno, and F. J. Garcia-Vidal, 2008, Phys. Rev. Lett. 101, 014301.

Chu, J., T.-J. Wang, J.-T. Yeh, M.-W. Lin, Y.-C. Chang, and J.-K. Wang, 2007, Appl. Phys. A: Mater. Sci. Process. 89, 387. Coe, J. V., J. M. Heer, S. Teeters-Kennedy, H. Tian, and K. R. Rodriguez, 2008, Annu. Rev. Phys. Chem. 59, 179.

Coe, J. V., S. M. Williams, K. R. Rodriguez, S. TeetersKennedy, S. A., and F. Hrovat, 2006, Anal. Chem. 78, 1385. Cohn, S. B., 1952, Proc. IRE 40, 696.

Colas des Francs, G., D. Molenda, U. C. Fischer, and A. 
Naber, 2005, Phys. Rev. B 72, 165111.

Collin, R. E., and W. H. Eggimann, 1961, IRE Trans. Microwave Theory Tech. 9, 110.

Collin, S., F. Pardo, and J.-L. Pelouard, 2003, Appl. Phys. Lett. 83, 1521.

Collin, S., F. Pardo, R. Teissier, and J. L. Pelouard, 2001, Phys. Rev. B 63, 331071.

Collin, S., F. Pardo, R. Teissier, and J. L. Pelouard, 2002, J. Opt. A, Pure Appl. Opt. 4, S154.

Collin, S., F. Pardo, R. Teissier, and J.-L. Pelouard, 2004, Appl. Phys. Lett. 85, 194.

Crouse, D., and P. Keshavareddy, 2005, Opt. Express 13, 7760.

Ctitis, G., P. Patoka, X. Wang, K. Kempa, and M. Giersig, 2007, Nano Lett. 7, 2926.

Dahlin, A., M. Zach, T. Rindzevius, M. Kall, D. S. Sutherland, and F. Hook, 2005, J. Am. Chem. Soc. 127, 5043.

Darmanyan, S. A., M. Neviere, and A. V. Zayats, 2004, Phys. Rev. B 70, 075103.

De Abajo, F. J. G., 2002, Opt. Express 10, 1475.

De Abajo, F. J. G., 2007, Rev. Mod. Phys. 79, 1267.

De Abajo, F. J. G., R. Gomez-Medina, and J. J. Saenz, 2005, Phys. Rev. E 72, 016608.

De Abajo, F. J. G., and J. J. Saenz, 2005, Phys. Rev. Lett. 95, 233901.

Degiron, A., and T. W. Ebbesen, 2004, Opt. Express 12, 3694. Degiron, A., and T. W. Ebbesen, 2005, J. Opt. A, Pure Appl. Opt. 7, S90.

Degiron, A., H. J. Lezec, W. L. Barnes, and T. W. Ebbesen, 2002, Appl. Phys. Lett. 81, 4327.

Degiron, A., H. J. Lezec, N. Yamamoto, and T. W. Ebbesen, 2004, Opt. Commun. 239, 61.

De Leebeeck, A., L. K. S. Kumar, V. De Lange, D. Sinton, R. Gordon, and A. G. Brolo, 2007, Anal. Chem. 79, 4094.

de Leon-Perez, F., G. Brucoli, F. J. Garcia-Vidal, and L. Martin-Moreno, 2008, New J. Phys. 10, 105017.

Dennis, M. R., N. I. Zheludev, and F. J. G. de Abajo, 2007, Opt. Express 15, 9692.

Devaux, E., T. W. Ebbesen, J.-C. Weeber, and A. Dereux, 2003, Appl. Phys. Lett. 83, 4936.

Dickson, W., G. A. Wurtz, P. R. Evans, R. J. Pollard, and A. V. Zayats, 2008, Nano Lett. 8, 281.

DiMaio, J. R., and J. Ballato, 2006, Opt. Express 14, 2380.

Dintinger, J., S. Klein, and T. W. Ebbesen, 2006, Adv. Mater. 18, 1267.

Dintinger, J., I. Robel, P. V. Kamat, C. Genet, and T. W. Ebbesen, 2006, Adv. Mater. 18, 1645.

Docter, M. W., 2008, Ph.D. thesis (Technical University Delft).

Docter, M. W., P. M. van der Berg, P. F. A. Alkemade, V. G. Kutchoukov, O. M. Piciu, I. T. Young, and Y. Garini, 2007, J. Nanophotonics 1, 011665.

Docter, M. W., I. T. Young, O. M. Piciu, A. Bossche, P. F. A. Alkemade, P. M. van den Berg, and Y. Garini, 2006, Opt. Express 14, 9477.

Dogariu, A., A. Nahata, R. A. Linke, L. J. Wang, and R. Trebino, 2002, Appl. Phys. B: Lasers Opt. 74, S69.

Dogariu, A., T. Thio, L. J. Wang, T. W. Ebbesen, and H. J. Lezec, 2001, Opt. Lett. 26, 450.

Donev, E. U., J. Y. Suh, F. Villegas, R. Lopez, R. F. Haglund, and L. C. Feldman, 2006, Phys. Rev. B 73, 201401.

Drexhage, K. H., 1974, Prog. Opt. 12, 165.

Drezet, A., C. Genet, and T. W. Ebbesen, 2008, Phys. Rev. Lett. 101, 043902.

Dykhne, A. M., A. K. Sarychev, and V. M. Shalaev, 2003, Phys.
Rev. B 67, 195402.

Ebbesen, T. W., C. Genet, and S. I. Bozhevolnyi, 2008, Phys. Today 61 (5), 44.

Ebbesen, T. W., H. J. Lezec, H. F. Ghaemi, T. Thio, and P. A. Wolff, 1998, Nature (London) 391, 667.

Edel, J. B., M. Wu, B. Baird, and H. G. Craighead, 2005, Biophys. J. 88, L43.

Eggiman, W. H., and R. E. Collin, 1962, IRE Trans. Microwave Theory Tech. 10, 528.

Ekinci, Y., H. H. Solak, and C. David, 2007, Opt. Lett. 32, 172. Elliott, J., I. I. Smolyaninov, N. I. Zheludev, and A. V. Zayats, 2004, Opt. Lett. 29, 1414.

Elliott, J., I. I. Smolyaninov, N. I. Zheludev, and A. V. Zayats, 2005, Phys. Rev. B 70, 233403.

Emerson, D. T., 1997, IEEE Trans. Microwave Theory Tech. 45, 2267.

Enoch, E., E. Popov, N. M., and R. R., 2002, J. Opt. A, Pure Appl. Opt. 4, S83.

Estrada, H., P. Candelas, A. Uris, F. Belmar, F. Meseguer, and F. J. G. de Abajo, 2008, Appl. Phys. Lett. 93, 011907.

Fan, W. J., S. Zhang, K. J. Malloy, and S. R. J. Brueck, 2005a, Opt. Express 13, 4406.

Fan, W. J., S. Zhang, K. J. Malloy, and S. R. J. Brueck, 2005b, J. Vac. Sci. Technol. B 23, 2700.

Fan, W. J., S. A. Zhang, K. J. Malloy, S. R. J. Brueck, N. C. Panoiu, and R. M. Osgood, 2006, Opt. Express 14, 9570.

Fan, W. J., S. Zhang, B. Minhas, K. J. Malloy, and S. R. J. Brueck, 2005, Phys. Rev. Lett. 94, 033902.

Fan, W. J., S. Zhang, N. C. Panoiu, A. Abdenour, S. Krishna, R. M. Osgood, K. J. Malloy, and S. R. J. Brueck, 2006, Nano Lett. 6, 1027.

Fang, X., Z. Y. Li, Y. B. Long, H. X. Wei, R. J. Liu, J. Y. Ma, M. Kamran, H. Y. Zhao, X. F. Han, and B. R. Zhao, 2007, Phys. Rev. Lett. 99, 066805.

Fano, U., 1961, Phys. Rev. 124, 1866.

Farcau, C., and S. Astilean, 2007, J. Opt. A, Pure Appl. Opt. 9, S345.

Felsen, L. P., and N. Marcuvitz, 2003, Radiation and Scattering of Waves (Wiley-Interscience, New York).

Feng, L., and P. Dawson, 2007, Opt. Express 15, 17863.

Fernandez-Dominguez, A. I., F. J. Garcia-Vidal, and L. Martin-Moreno, 2007, Phys. Rev. B 76, 235430.

Fernandez-Dominguez, A. I., D. Martin-Cano, L. MartinMoreno, and F. J. Garcia-Vidal, 2008, Phys. Rev. A 78, 023614.

Fernandez-Dominguez, A. I., E. Moreno, L. Martin-Moreno, and F. J. Garcia-Vidal, 2006, Phys. Rev. A 74, 021601(R).

Fong, K. Y., and P. M. Hui, 2006, Appl. Phys. Lett. 89, 091101. Frei, W. R., D. A. Tortorelli, and H. T. Johnson, 2005, Appl. Phys. Lett. 86, 111114.

Galindo, V., and C. P. Wu, 1966, IEEE Trans. Antennas Propag. AP-14, 149.

Gao, D., W. Chen, and A. Mulchandani, 2007, Appl. Phys. Lett. 90, 073901.

Gao, H. W., J. Henzie, and T. W. Odom, 2006, Nano Lett. 6, 2104.

Garcia-Vidal, F. J., H. J. Lezec, T. W. Ebbesen, and L. MartinMoreno, 2003, Phys. Rev. Lett. 90, 213901.

Garcia-Vidal, F. J., and L. Martin-Moreno, 2002, Phys. Rev. B 66, 155412.

Garcia-Vidal, F. J., L. Martin-Moreno, H. J. Lezec, and T. W. Ebbesen, 2003, Appl. Phys. Lett. 83, 4500.

Garcia-Vidal, F. J., L. Martin-Moreno, E. Moreno, L. K. S. 
Kumar, and R. Gordon, 2006, Phys. Rev. B 74, 153411.

Garcia-Vidal, F. J., L. Martin-Moreno, and J. B. Pendry, 2005, J. Opt. A, Pure Appl. Opt. 7, S97.

Garcia-Vidal, F. J., E. Moreno, J. A. Porto, and L. MartinMoreno, 2005, Phys. Rev. Lett. 95, 103901.

Garcia-Vidal, F. J., S. G. Rodrigo, and L. Martin-Moreno, 2006, Nat. Phys. 2, 790.

Gay, G., B. V. de Lesegno, R. Mathevet, J. Weiner, H. J. Lezec, and T. W. Ebbesen, 2005, Appl. Phys. B: Lasers Opt. 81, 871.

Genet, C., M. P. Altewischer, E. van Exter, and J. P. Woerdman, 2005, Phys. Rev. B 71, 033409.

Genet, C., and T. Ebbesen, 2007, Nature (London) 445, 39.

Genet, C., M. P. van Exter, and J. P. Woerdman, 2003, Opt. Commun. 225, 331.

Gérard, D., V. Laude, B. Sadani, A. Khelif, D. Van Labeke, and B. Guizal, 2007, Phys. Rev. B 76, 235427.

Ghaemi, H. F., T. Thio, D. E. Grupp, T. W. Ebbesen, and H. J. Lezec, 1998, Phys. Rev. B 58, 6779.

Gomez Rivas, J., P. H. Janke, C. Bolivar, and H. Kurz, 2005, Opt. Express 13, 847.

Gomez Rivas, J., C. Schotsch, P. H. Bolivar, and H. Kurz, 2003, Phys. Rev. B 68, 201306.

Gordon, R., 2007, Phys. Rev. A 76, 053806.

Gordon, R., and A. G. Brolo, 2005, Opt. Express 13, 1933.

Gordon, R., A. G. Brolo, A. McKinnon, A. Rajora, B. Leathem, and K. L. Kavanagh, 2004, Phys. Rev. Lett. 92, 037401.

Gordon, R., M. Hughes, B. Leathem, K. L. Kavanagh, and A. G. Brolo, 2005, Nano Lett. 5, 1243.

Gordon, R., D. Sinton, K. L. Kavanagh, and A. G. Brolo, 2008, Acc. Chem. Res. 41, 1049.

Groeneveld, G. H. M., R. Sprik, and A. Lagendijk, 1990, Phys. Rev. Lett. 64, 784.

Gruhlke, R., W. Holland, and D. Hall, 1986, Phys. Rev. Lett. 56, 2838.

Grupp, D. E., H. J. Lezec, T. W. Ebbesen, K. M. Pellerin, and T. Thio, 2000, Appl. Phys. Lett. 77, 1569.

Guo, B., G. Song, and L. Chen, 2007, Appl. Phys. Lett. 91, 021103.

Guo, B., G. Song, and L. Chen, 2008, Appl. Phys. B: Lasers Opt. 91, 7.

Guo, G.-P., X.-F. Ren, Y.-F. Huang, C.-F. Li, Z.-Y. Ou, and G.-C. Guo, 2007, Phys. Lett. A 361, 218.

Haftel, M. I., C. Schlockermann, and G. Blumberg, 2006, Phys. Rev. B 74, 235405.

Halte, V., A. Benabbas, L. Guidoni, and J. Y. Bigot, 2005, Phys. Status Solidi B 242, 1872.

Harrington, R. F., and D. T. Auckland, 1980, IEEE Trans. Antennas Propag. AP-28, 616.

Hashizume, J., and F. Koyama, 2004, Appl. Phys. Lett. 84, 3226.

Hashizume, J., and F. Koyama, 2007, Electron. Commun. Jpn., Part 2: Electron. 90, 49.

Hendry, E., F. J. Garcia-Vidal, L. Martin-Moreno, J. Gomez Rivas, M. Bonn, A. P. Hibbins, and M. J. Lockyear, 2008, Phys. Rev. Lett. 100, 123901.

Hendry, E., J. R. Hibbins, and A. P. Sambles, 2008, Phys. Rev. B 78, 235426.

Hendry, E., M. J. Lockyear, J. Gomez-Rivas, L. Kuipers, and M. Bonn, 2007, Phys. Rev. B 75, 235305.

Henzie, J., M. Lee, and T. Odom, 2007, Nat. Nanotechnol. 2, 549 .

Hessel, A., and A. A. Oliner, 1965, Appl. Opt. 4, 1275.
Hibbins, A. P., B. R. Evans, and J. R. Sambles, 2005, Science 308, 670 .

Hibbins, A. P., I. R. Hooper, M. J. Lockyear, and J. R. Sambles, 2006, Phys. Rev. Lett. 96, 257402.

Hibbins, A. P., J. R. Sambles, and C. R. Lawrence, 2002, Appl. Phys. Lett. 81, 4661.

Hibbins, A. P., J. R. Sambles, C. R. Lawrence, and D. M. Robinson, 2001, Appl. Phys. Lett. 79, 2844.

Hohng, S. C., D. S. Kim, Y. Yoon, V. Malarchuk, C. Lienau, J. W. Park, K. H. Yoo, J. Kim, S. H. Han, and Q. H. Park, 2005, J. Korean Phys. Soc. 46, S205.

Hohng, S. C., Y. Yoon, D. S. Kim, V. Malarchuk, R. Muller, C. Lienau, J. W. Park, K. H. Yoo, J. Kim, H. Y. Ryu, and Q. H. Park, 2002, Appl. Phys. Lett. 81, 3239.

Hongo, K., 1971, Electron. Commun. Jpn. 54-B, 90.

Hooper, I. R., and J. R. Sambles, 2003, Phys. Rev. B 67, 235404.

Hou, B., Z. H. Hang, W. J. Wen, C. T. Chan, and P. Sheng, 2006, Appl. Phys. Lett. 89, 131917.

Hsu, S.-Y., M.-C. Lee, K.-L. Lee, and P.-K. Wei, 2008, Appl. Phys. Lett. 92, 013303.

Huang, C., C. Du, and X. Luo, 2007, Appl. Phys. Lett. 91, 143512.

Huang, F. M., N. I. Zheludev, Y. F. Chen, and F. J. G. de Abajo, 2007, Appl. Phys. Lett. 90, 091119.

Ishi, T., J. Fujikata, K. Makita, T. Baba, and K. Ohashi, 2005, Jpn. J. Appl. Phys., Part 2 44, L364.

Ishi, T., J. Fujikata, and K. Ohashi, 2005, Jpn. J. Appl. Phys., Part 2 44, L170.

Ishihara, K., G. Hatakoshi, T. Ikari, H. Minamide, H. Ito, and K. Ohashi, 2005, Jpn. J. Appl. Phys., Part 2 44, L1005.

Ishihara, K., T. Ikari, H. Minamide, J. Shikata, K. Ohashi, H. Yokoyama, and H. Ito, 2005, J. Appl. Phys. 44, L929.

Ishihara, K., K. Ohashi, T. Ikari, H. Minamide, H. Yokoyama, J. Shikata, and H. Ito, 2006, Appl. Phys. Lett. 89, 201120.

Jeanmaire, D. L., and R. P. Van Duyne, 1977, J. Electroanal. Chem. 84, 1.

Ji, J., J. G. O'Connell, D. J. D. Carter, and D. N. Larson, 2008, Anal. Chem. 80, 2491.

Kashyap, S. C., and M. A. K. Hamid, 1971, IEEE Trans. Antennas Propag. AP-19, 499.

Kats, A. V., M. L. Nesterov, and A. Y. Nikitin, 2005, Phys. Rev. B 72, 193405.

Kats, A. V., and A. Y. Nikitin, 2004, Phys. Rev. B 70, 235412.

Keilmann, F., 1981, Int. J. Infrared Millim. Waves 2, 259.

Kihm, H. W., K. G. Lee, D. S. Kim, J. H. Kang, and Q.-H. Park, 2008, Appl. Phys. Lett. 92, 051115.

Kim, D. S., S. C. Hohng, V. Malyarchuk, Y. C. Yoon, Y. H. Ahn, K. J. Yee, J. W. Park, J. Kim, Q. H. Park, and C. Lienau, 2003, Phys. Rev. Lett. 91, 143901.

Kim, J. H., and P. J. Moyer, 2006a, Opt. Express 14, 6595.

Kim, J. H., and P. J. Moyer, 2006b, Appl. Phys. Lett. 89, 121106.

Kim, S., H. Kim, Y. Lim, and B. Lee, 2007, Appl. Phys. Lett. 90, 051113.

Kim, S., Y. Lim, H. Kim, J. Park, and B. Lee, 2008, Appl. Phys. Lett. 92, 013103.

Kim, T. J., T. Thio, T. W. Ebbesen, D. E. Grupp, and H. J. Lezec, 1999, Opt. Lett. 24, 256.

Koerkamp, K. J. K., S. Enoch, F. B. Segerink, N. F. van Hulst, and L. Kuipers, 2004, Phys. Rev. Lett. 92, 183901.

Korobkin, D., Y. A. Urzhumov, B. Neuner, Z. Zhang, I. D. Mayergoyz, and G. Shvets, 2007, Appl. Phys. A: Mater. Sci. 
Process. 88, 605.

Kramper, P., M. Agio, C. M. Soukoulis, A. Birner, F. Muller, R. B. Wehrspohn, U. Gosele, and V. Sandoghdar, 2004, Phys. Rev. Lett. 92, 113903.

Krishnan, A., T. Thio, T. J. Kim, H. J. Lezec, T. W. Ebbesen, P. A. Wolff, J. Pendry, L. Martin-Moreno, and F. J. GarciaVidal, 2001, Opt. Commun. 200, 1.

Kumar, L. K. S., and R. Gordon, 2006, IEEE J. Sel. Top. Quantum Electron. 12, 1228.

Lalanne, P., and J. P. Hogonin, 2006, Nat. Phys. 2, 551.

Lalanne, P., J. P. Hogonin, S. Astilean, M. Palamaru, and K. D. Moller, 2000, J. Opt. A, Pure Appl. Opt. 2, 48.

Lalanne, P., J. P. Hugonin, and J. C. Rodier, 2005, Phys. Rev. Lett. 95, 263902.

Lalanne, P., J. C. Rodier, and J. P. Hugonin, 2005, J. Opt. A, Pure Appl. Opt. 7, 422.

Laluet, J.-Y., E. Devaux, C. Genet, T. W. Ebbesen, J.-C. Weeber, and A. Dereux, 2007, Opt. Express 15, 3488.

Landau, L. D., E. M. Lifshitz, and L. P. Pitaevskii, 1960, Electrodynamics of Continuous Media (Pergamon, New York).

Landstrom, L., D. Brodoceanu, D. Bauerle, F. J. Garcia-Vidal, S. G. Rodrigo, and L. Martin-Moreno, 2009, Opt. Express 17, 761.

Landstrom, L., D. Brodoceanu, K. Piglmayer, G. Langer, and D. Bauerle, 2005, Appl. Phys. A: Mater. Sci. Process. 81, 15.

Laux, E., C. Genet, T. Skauli, and T. W. Ebbesen, 2008, Nat. Photonics 2, 161.

Lee, J. W., M. A. Seo, D. H. Kang, K. S. Khim, S. C. Jeoung, and D. S. Kim, 2007, Phys. Rev. Lett. 99, 137401.

Lee, J. W., M. A. Seo, D. J. Park, D. S. Kim, C. Lienau, S. C. Jeoung, Q. H. Park, and P. C. M. Planken, 2006, Opt. Express 14, 1253.

Lee, J. W., M. A. Seo, J. Y. Sohn, Y. H. Ahn, D. S. Kim, S. C. Jeoung, C. Lienau, and Q.-H. Park, 2005, Opt. Express 13, 10681.

Lee, K. G., and Q.-H. Park, 2005, Phys. Rev. Lett. 95, 103902.

Lee, P. S. K., M. P. van Exter, and J. P. Woerdman, 2006, J. Opt. Soc. Am. B 23, 134.

Lehman, G. W., 1970, J. Math. Phys. 11, 1522.

Lesuffleur, A., H. Im, N. C. Lindquist, K. S. Lim, and S.-H. Oh, 2008, Opt. Express 16, 219.

Lesuffleur, A., L. K. S. Kumar, A. G. Brolo, K. L. Kavanagh, and R. Gordon, 2007, J. Phys. Chem. C 111, 2347.

Lesuffleur, A., L. K. S. Kumar, and R. Gordon, 2007, Phys. Rev. B 75, 045423.

Levene, M. J., J. Korlach, S. W. Turner, M. Fouquet, H. G. Craighead, and W. W. Webb, 2003, Science 299, 682.

Lewis, A., M. Isaacson, A. Harootunian, and A. Murray, 1984, Ultramicroscopy 13, 227.

Lezec, H. J., A. Degiron, E. Devaux, R. A. Linke, L. MartinMoreno, F. J. Garcia-Vidal, and T. W. Ebbesen, 2002, Science 297, 820 .

Lezec, H. J., and T. Thio, 2004, Opt. Express 12, 3629.

Li, L., 1997, J. Opt. Soc. Am. A 14, 2758.

Li, T., H. Liu, F. M. Wang, Z. G. Dong, S. N. Zhu, and X. Zhang, 2006, Opt. Express 14, 11155.

Li, Z., K. Aydin, and E. Ozbay, 2007, Appl. Phys. Lett. 91, 121105.

Li, Z., H. Caglayan, E. Colak, and E. Ozbay, 2008, Appl. Phys. Lett. 92, 011128.

Lin, D. Z., C. K. Chang, Y. C. Chen, D. L. Yang, M. W. Lin, J. T. Yeh, J. M. Liu, C. H. Kuan, C. S. Yeh, and C. K. Lee, 2006, Opt. Express 14, 3503.
Lin, D. Z., T. D. Cheng, C. K. Chang, J. T. Yeh, J. M. Liu, C. S. Yeh, and C. K. Lee, 2007, Opt. Express 15, 2585.

Liu, C., V. Kamaev, and Z. V. Vardeny, 2005, Appl. Phys. Lett. 86, 143501.

Liu, H., and P. Lalanne, 2008, Nature (London) 452, 728.

Liu, W. C., and D. P. Tsai, 2002, Phys. Rev. B 65, 155423.

Liu, Y., J. Bishop, L. Williams, S. Blair, and J. Herron, 2004, Nanotechnology 15, 1368.

Liu, Y., and S. Blair, 2003, Opt. Lett. 28, 507.

Liu, Y., and S. Blair, 2004, Opt. Express 12, 3686.

Lockyear, M. J., A. P. Hibbins, J. R. Sambles, and C. R. Lawrence, 2004, Appl. Phys. Lett. 84, 2040.

Lockyear, M. J., A. P. Hibbins, J. R. Sambles, and C. R. Lawrence, 2005, J. Opt. A, Pure Appl. Opt. 7, S152.

Lomakin, V., S. Q. Li, and E. Michielssen, 2007, Microwave Opt. Technol. Lett. 49, 1554.

López-Tejeira, F., F. J. Garcia-Vidal, and L. Martin-Moreno, 2005, Phys. Rev. B 72, 161405.

López-Tejeira, F., S. G. Rodrigo, L. Martin-Moreno, F. J. Garcia-Vidal, E. Devaux, T. W. Ebbesen, J. R. Krenn, I. P. Radko, S. I. Bozhevolnyi, M. U. Gonzalez, J.-C. Weeber, and A. Dereux, 2007, Nat. Phys. 3, 324.

Lu, M.-H., X.-K. Liu, L. Feng, J. Li, C.-P. Huang, Y.-F. Chen, Y.-Y. Zhu, S.-N. Zhu, and N.-B. Ming, 2007, Phys. Rev. Lett. 99, 174301.

Luo, X., and T. Ishihara, 2004, Appl. Phys. Lett. 64, 4780.

Maier, S. A., 2007, Plasmonics: Fundamentals and Applications (Springer, New York).

Marquier, F., J. J. Greffet, S. Collin, F. Pardo, and J. L. Pelouard, 2005, Opt. Express 13, 70.

Marquier, F., K. Joulain, and J. J. Greffet, 2004, Opt. Lett. 29, 2178.

Martin-Moreno, L., F. J. Garcia-Vidal, H. J. Lezec, A. Degiron, and T. W. Ebbesen, 2003, Phys. Rev. Lett. 90, 167401.

Martin-Moreno, L., F. J. Garcia-Vidal, H. J. Lezec, K. M. Pellerin, T. Thio, J. B. Pendry, and T. W. Ebbesen, 2001, Phys. Rev. Lett. 86, 1114.

Mary, A., S. G. Rodrigo, F. J. Garcia-Vidal, and L. MartinMoreno, 2008, Phys. Rev. Lett. 101, 103902.

Mary, A., S. G. Rodrigo, L. Martin-Moreno, and F. J. GarciaVidal, 2007, Phys. Rev. B 76, 195414.

Masson, J. B., and G. Gallot, 2006, Phys. Rev. B 73, 121401.

Matsui, T., A. Agrawal, A. Nahata, and Z. V. Vardeny, 2007, Nature (London) 446, 517.

Matsui, T., Z. V. Verdeny, A. Agrawal, A. Nahata, and R. Menon, 2006, Appl. Phys. Lett. 88, 071101.

McPhedran, R. C., and D. Maystre, 1977, Appl. Phys. 14, 1.

Medina, F., F. Mesa, and R. Marqués, 2008, IEEE Trans. Microwave Theory Tech. 56, 3108.

Mei, J., B. Hou, M. Ke, S. Peng, H. Jia, Z. Liu, J. Shi, W. Wen, and P. Sheng, 2008, Appl. Phys. Lett. 92, 124106.

Min, C., P. Wang, X. Jiao, Y. Deng, and H. Ming, 2008, Appl. Phys. B: Lasers Opt. 90, 97.

Min, C. J., P. Wang, X. J. Jiao, Y. Deng, and H. Ming, 2007, Opt. Express 15, 12368.

Mitsuishi, A., Y. Otsuka, S. Fujita, and H. Yoshinaga, 1963, Jpn. J. Appl. Phys. 2, 574.

Miyamaru, F., and M. Hangyo, 2004, Appl. Phys. Lett. 84, 2742.

Miyamaru, F., and M. Hangyo, 2005, Phys. Rev. B 71, 165408. Miyamaru, F., T. Kondo, T. Nagashima, and M. Hangyo, 2003, Appl. Phys. Lett. 82, 2568.

Miyamaru, F., M. Tanaka, and M. Hangyo, 2006a, Phys. Rev. B 
74, 153416.

Miyamaru, F., M. Tanaka, and M. Hangyo, 2006b, Phys. Rev. B 74, 115117.

Moerland, R. J., N. F. van Hulst, H. Gersen, and L. Kuipers, 2005, Opt. Express 13, 1604.

Moreau, A., G. Granet, F. I. Baida, and D. Van Labeke, 2003, Opt. Express 11, 1131.

Moreno, E., 2008, private communication.

Moreno, E., A. I. Fernandez-Dominguez, J. I. Cirac, F. J. Garcia-Vidal, and L. Martin-Moreno, 2005, Phys. Rev. Lett. 95, 170406.

Moreno, E., F. J. Garcia-Vidal, D. Erni, J. I. Cirac, and L. Martin-Moreno, 2004, Phys. Rev. Lett. 92, 236801.

Moreno, E., F. J. Garcia-Vidal, and L. Martin-Moreno, 2004, Phys. Rev. B 69, 121402(R).

Moreno, E., L. Martin-Moreno, and F. J. Garcia-Vidal, 2004, Photonics Nanostruct. Fundam. Appl. 2, 97.

Morrison, S. K., and Y. S. Kivshar, 2005, Appl. Phys. Lett. 86, 081110.

Moussa, R., B. Wang, G. Tuttle, T. Koschny, and C. M. Soukoulis, 2007, Phys. Rev. B 76, 235417.

Mrejen, M., A. Israel, H. Taha, M. Palchan, and A. Lewis, 2007, Opt. Express 15, 9129.

Muller, R., V. Malyarchuk, and C. Lienau, 2003, Phys. Rev. B 68, 205415.

Müller, R., C. Ropers, and C. Lienau, 2004, Opt. Express 12, 5067.

Munk, B. A., 2000, Frequency Selective Surfaces: Theory and Design (Wiley Interscience, New York).

Nahata, A., R. A. Linke, T. Ishi, and K. Ohashi, 2003, Opt. Lett. 28, 423.

Naweed, A., F. Baumann, W. A. Bailey, A. S. Karakashian, and W. D. Goodhue, 2003, J. Opt. Soc. Am. B 20, 2534.

Neerhoff, F. L., and G. Mur, 1973, Appl. Sci. Res. 28, 73.

Neviere, M., 1980, Electromagnetic Theory of Gratings (Springer-Verlag, Berlin).

Nikitin, A. Y., G. Brucoli, F. J. Garcia-Vidal, and L. MartinMoreno, 2008, Phys. Rev. B 77, 195441.

Nikitin, A. Y., D. Zueco, F. J. Garcia-Vidal, and L. MartinMoreno, 2008, Phys. Rev. B 78, 165429.

Nylander, C., B. Liedberg, and T. Lind, 1982, Sens. Actuators 3, 79 .

Obermuller, C., and K. Karrai, 1995, Appl. Phys. Lett. 67, 3408.

Ohno, T., J. A. Bain, and T. E. Schlesinger, 2007, J. Appl. Phys. 101, 083107.

Onishi, T., T. Tanigawa, T. Ueda, and D. Ueda, 2007, IEEE J. Quantum Electron. 43, 1123.

Orbons, S. M., and A. Roberts, 2006, Opt. Express 14, 12623.

Orbons, S. M., A. Roberts, D. N. Jamieson, M. I. Haftel, C. Schlockermann, D. Freeman, and B. Luther-Davies, 2007, Appl. Phys. Lett. 90, 251107.

Ozbay, E., 2006, Science 311, 189.

Palik, E. D., 1985, Handbook of Optical Constants of Solids (Academic, New York).

Pang, Y., C. Genet, and T. W. Ebbesen, 2007, Opt. Commun. 280, 10.

Papasimakis, N., V. A. Fedotov, A. S. Schwanecke, N. I. Zheludev, and F. J. G. De Abajo, 2007, Appl. Phys. Lett. 91, 081503.

Park, T.-H., N. Mirin, J. B. Lassiter, C. L. Nehl, N. J. Halas, and P. Nordlander, 2008, ACS Nano 2, 25.

Parthasarathy, R., A. Bykhovski, B. Gelmont, T. Globus, N.
Swami, and D. Woolard, 2007, Phys. Rev. Lett. 98, 153906.

Pendry, J. B., and A. MacKinnon, 1992, Phys. Rev. Lett. 69, 2772.

Pendry, J. B., L. Martin-Moreno, and F. J. Garcia-Vidal, 2004, Science 305, 847.

Pitarke, J. M., V. M. Silkin, E. V. Chulkov, and P. M. Echenique, 2007, Rep. Prog. Phys. 70, 1.

Pohl, D. W., W. Denk, and M. Lanz, 1984, Appl. Phys. Lett. 44, 651.

Popov, E., N. Bonod, M. Neviere, H. Rigneault, P.-F. Lenne, and P. Chaumet, 2005, Appl. Opt. 44, 2332.

Popov, E., M. Neviere, S. Enoch, and R. Reinisch, 2000, Phys. Rev. B 62, 16100.

Porto, J. A., F. J. Garcia-Vidal, and J. B. Pendry, 1999, Phys. Rev. Lett. 83, 2845.

Porto, J. A., L. Martin-Moreno, and F. J. Garcia-Vidal, 2004, Phys. Rev. B 70, 081402.

Poujet, Y., M. Roussey, J. Salvi, F. I. Baida, D. Van Labeke, A. Perentes, C. Santischi, and P. Hoffmann, 2006, Photonics Nanostruct. Fundam. Appl. 4, 47.

Prikulis, J., P. Hanarp, L. Olofsson, D. Sutherland, and M. Kall, 2004, Nano Lett. 4, 1003.

Przybilla, F., A. Degiron, C. Genet, T. W. Ebbesen, F. de LeonPerez, J. Bravo-Abad, F. Garcia-Vidal, and L. MartinMoreno, 2008, Opt. Express 16, 9571.

Przybilla, F., A. Degiron, J. Y. Laluet, C. Genet, and T. W. Ebbesen, 2006, J. Opt. A, Pure Appl. Opt. 8, 458.

Przybilla, F., C. Genet, and T. W. Ebbesen, 2006, Appl. Phys. Lett. 89, 121115.

Qu, D., and D. Grischkowsky, 2004, Phys. Rev. Lett. 93, 196804.

Reilly, T. H., S.-H. Chang, J. D. Corbman, G. C. Schatz, and K. L. Rowlen, 2007, J. Phys. Chem. C 111, 1689.

Reilly, T. H. I., and K. L. Rowlen, 2004, Proc. SPIE 5513, 250.

Ren, X.-F., G.-P. Guo, P. Zhang, Y.-F. Huang, Z.-W. Wang, and G.-C. Guo, 2007, Appl. Phys. B: Lasers Opt. 89, 257.

Renk, R. W., and L. Gensel, 1962, Appl. Opt. 1, 643.

Rhoads, C. M., E. K. Damon, and B. A. Munk, 1982, Appl. Opt. 21, 2814.

Rignault, H., J. Capoulade, J. Dintinger, J. Wenger, N. Bonod, E. Popov, T. W. Ebbesen, and P.-F. Lenne, 2005, Phys. Rev. Lett. 95, 117401.

Rindzevicius, T., Y. Alaverdyan, A. Dahlin, F. Hook, D. S. Sutherland, and M. Kall, 2005, Nano Lett. 5, 2535.

Ritchie, R. H., 1957, Phys. Rev. 106, 874.

Roberts, A., 1987, J. Opt. Soc. Am. A 4, 1970.

Rockstuhl, C., F. Lederer, T. Zentgraf, and H. Giessen, 2007, Appl. Phys. Lett. 91, 151109.

Rodrigo, S. G., F. J. Garcia-Vidal, and L. Martin-Moreno, 2008, Phys. Rev. B 77, 075401.

Rokitski, R., K. A. Tetz, and Y. Fainman, 2005a, Phys. Rev. Lett. 95, 177401.

Rokitski, R., K. A. Tetz, and Y. Fainman, 2005b, Nano Lett. 5, 1963.

Ropers, C., D. J. Park, G. Stibenz, G. Steinmeyer, D. S. Kim, and C. Lienau, 2005, Phys. Rev. Lett. 94, 113901.

Ropers, C., G. Stibenz, G. Steinmeyer, R. Muller, D. J. Park, K. G. Lee, J. E. Kihm, J. Kim, Q. H. Park, D. S. Kim, and C. Lienau, 2006, Appl. Phys. B: Lasers Opt. 84, 183.

Salomon, L., F. Grillot, A. V. Zayats, and F. De Fornel, 2001, Phys. Rev. Lett. 86, 1110.

Salvi, J., et al., 2005, Opt. Lett. 30, 1611.

Sarrazin, M., and J. P. Vigneron, 2003, Phys. Rev. E 68, 166031. 
Sarrazin, M., and J. P. Vigneron, 2004, Opt. Commun. 240, 89. Sarrazin, M., J. P. Vigneron, and J. M. Vigoureux, 2003, Phys. Rev. B 67, 085415.

Schellenberg, F. M., K. Adam, J. Matteo, and L. Hessenlink, 2005, J. Vac. Sci. Technol. B 23, 3106.

Schouten, H. F., N. Kuzmin, G. Dubois, T. D. Visser, G. Gbur, P. F. A. Alkemade, H. Blok, G. W. 't Hooft, D. Lenstra, and E. R. Eliel, 2005, Phys. Rev. Lett. 94, 053901.

Schouten, H. F., T. D. Visser, D. Lenstra, and H. Blok, 2003, Phys. Rev. E 67, 036608.

Schroter, U., and D. Heitmann, 1998, Phys. Rev. B 58, 15419.

Seo, M. A., H. R. Park, S. M. Koo, D. J. Park, J. H. Kang, O. K. Suwal, S. S. Choi, P. C. M. Planken, G. S. Park, N. K. Park, Q.-H. Park, and D. S. Kim, 2009, Nat. Photonics 3, 152.

Seo, S., H. C. Kim, H. Ko, and M. Cheng, 2007, J. Vac. Sci. Technol. B 25, 2271.

Sepúlveda, B., Y. Alaverdyan, J. Alegret, M. Käll, and P. Johansson, 2008, Opt. Express 16, 5609.

Shaner, E. A., J. G. Cederberg, and D. Wasserman, 2007, Appl. Phys. Lett. 91, 181110.

Shao, D. B., and S. C. Chen, 2005, Appl. Phys. Lett. 86, 253107.

Shao, D. B., and S. C. Chen, 2008, J. Vac. Sci. Technol. B 26, 227.

Sharpe, J. C., J. S. Mitchell, L. Lin, N. Sedoglavich, and R. J. Blaikie, 2008, Anal. Chem. 80, 2244.

Shen, J. T., P. B. Catrysse, and S. Fan, 2005, Phys. Rev. Lett. 94, 197401.

Shi, H., C. Du, and X. Luo, 2007, Appl. Phys. Lett. 91, 093111. Shinada, S., J. Hashizume, and F. Koyama, 2003, Appl. Phys. Lett. 83, 836.

Sick, B., B. Hecht, U. P. Wild, and L. Novotny, 2001, J. Microsc. 202, 365.

Sinton, D., R. Gordon, and A. G. Brolo, 2008, Microfluid. Nanofluid. 4, 107.

Skigin, D. C., and R. A. Depine, 2005, Phys. Rev. Lett. 95, 217402.

Skigin, D. C., and R. A. Depine, 2006, Opt. Commun. 262, 270.

Smolyaninov, I. I., A. V. Zayats, A. Stanishevsky, and C. C. Davis, 2002, Phys. Rev. B 66, 205414.

Sondergaard, T., and S. I. Bozhevolnyi, 2004, Phys. Rev. B 69, 045422.

Sonnichsen, C., A. C. Duch, G. Steininger, M. Koch, G. von Plessen, and J. Feldmann, 2000, Appl. Phys. Lett. 76, 140.

Srituravanich, W., N. Fang, S. Durant, M. Ambati, S. Sun, and X. Zhang, 2004, J. Vac. Sci. Technol. B 22, 3475.

Stark, P. R. H., A. E. Halleck, and D. N. Larson, 2005, Methods 37, 37.

Steele, J. M., C. E. Moran, A. Lee, C. M. Aguirre, and N. J. Halas, 2003, Phys. Rev. B 68, 205103.

Strelniker, Y. M., 2007, Phys. Rev. B 76, 085409.

Suckling, J. R., A. P. Hibbins, M. J. Lockyear, T. W. Preist, J. R. Sambles, and C. R. Lawrence, 2004, Phys. Rev. Lett. 92, 147401.

Suckling, J. R., A. P. Hibbins, J. R. Sambles, and C. R. Lawrence, 2005, New J. Phys. 7, 250.

Suh, J. Y., E. U. Donev, R. Lopez, L. C. Feldman, and R. F. Haglund, 2006, Appl. Phys. Lett. 88, 133115.

Sun, M., R.-J. Liu, Z.-Y. Li, B.-Y. Cheng, D.-Z. Zhang, H. F. Yang, and A. Z. Jin, 2006, Chin. Phys. 15, 1591.

Sun, M., R.-J. Liu, Z.-Y. Li, B.-Y. Cheng, D.-Z. Zhang, H. F. Yang, and A. Z. Jin, 2007, Phys. Lett. A 365, 510.

Sun, M., J. Tian, S. Z. Han, Z.-Y. Li, B.-Y. Cheng, D.-Z. Zhang, A. Z. Jin, and H. F. Yang, 2006, J. Appl. Phys. 100,
024320.

Sun, M., J. Tian, Z.-Y. Li, B.-Y. Cheng, D.-Z. Zhang, A. Z. Jin, and H. F. Yang, 2006, Chin. Phys. Lett. 23, 486.

Sun, Z., Y. Jung, and H. K. Kim, 2003, Appl. Phys. Lett. 83, 3021.

Sutherland, R. L., 1996, Handbook of Nonlinear Optics (Dekker, New York).

Takakura, Y., 2001, Phys. Rev. Lett. 86, 5601.

Tanaka, M., F. Miyamaru, M. Hangyo, T. Tanaka, M. Akazawa, and E. Sano, 2005, Opt. Lett. 30, 1210.

Tang, D., L. Chen, and W. Ding, 2006, Appl. Phys. Lett. 89, 131120.

Teeters-Kennedy, S., S. M. Williams, K. R. Rodriguez, K. Cilwa, D. Meleason, A. Sudnitsyn, F. Hrovat, and J. V. Coe, 2007, J. Phys. Chem. C 111, 124.

Tetz, K. A., L. Pang, and Y. Fainman, 2006, Opt. Lett. 31, 1528. Thio, T., H. F. Ghaemi, H. J. Lezec, P. A. Wolff, and T. W. Ebbesen, 1999, J. Opt. Soc. Am. B 16, 1743.

Thio, T., K. M. Pellerin, R. A. Linke, H. J. Lezec, and T. W. Ebbesen, 2001, Opt. Lett. 26, 1972.

Thomas, D. A., and H. P. Hughes, 2004, Solid State Commun. 129, 519.

Tranquilla, J. M., J. P. Carr, and H. M. Al-Rizzo, 1994, IEEE Trans. Antennas Propag. 42, 905.

Treacy, M. M. J., 1999, Appl. Phys. Lett. 75, 606.

Treacy, M. M. J., 2002, Phys. Rev. B 66, 195105.

Ulrich, R., 1967, Infrared Phys. 7, 37.

Ulrich, R., 1974, Microwave Res. Inst. Symp. Ser. 23, 359.

Ulrich, R., and M. Tacke, 1973, Appl. Phys. Lett. 22, 251.

Urzhumov, Y. A., D. Korobkin, B. Neuner, C. Zorman, and G. Shvets, 2007, J. Opt. A, Pure Appl. Opt. 9, S322.

van der Molen, K. L., K. J. Klein Koerkamp, F. B. Segerink, N. F. van Hulst, and L. Kuipers, 2005, Phys. Rev. B 72, 045421. van der Molen, K. L., F. B. Segerink, N. F. van Hulst, and L. Kuipers, 2004, Appl. Phys. Lett. 85, 4316.

van Exter, M., and A. Lagendijk, 1988, Phys. Rev. Lett. 60, 49. van Hulst, N. F., J. A. Veerman, M. F. Garcia-Parajo, and L. Kuipers, 2000, J. Chem. Phys. 112, 7799.

van Nieuwstadt, J. A. H., M. Sandtke, R. H. Harmsen, F. B. Segerink, J. C. Prangsma, S. Enoch, and L. Kuipers, 2006, Phys. Rev. Lett. 97, 146102.

Veerman, J. A., M. F. Garcia-Parajo, L. Kuipers, and N. F. van Hulst, 1999, J. Microsc. 194, 477.

Visser, T. D., 2006, Nat. Phys. 2, 509.

Wang, B., and G. P. Wang, 2006, Appl. Phys. Lett. 88, 013114. Wang, C., C. Du, and X. Luo, 2006, Phys. Rev. B 74, 245403. Wang, C., C. Du, Y. Lv, and X. Luo, 2006, Opt. Express 14, 5671.

Wannemacher, R., 2001, Opt. Commun. 195, 107.

Wasserman, D., E. A. Shaner, and J. G. Cederberg, 2007, Appl. Phys. Lett. 90, 191102.

Webb, K. J., and J. Li, 2006, Phys. Rev. B 73, 033401.

Wei, X., X. Luo, X. Duong, and C. Du, 2007, Opt. Express 15, 14177.

Wenger, J., D. Gerard, J. Dintinger, O. Mahboub, N. Bonod, E. Popov, T. W. Ebbesen, and H. Rignault, 2008, Opt. Express 16, 3008.

Went, H. E., A. P. Hibbins, J. R. Sambles, C. R. Lawrence, and A. P. Crick, 2000, Appl. Phys. Lett. 77, 2789.

Williams, C. R., S. R. Andrews, S. A. Maier, A. I. FernandezDominguez, L. Martin-Moreno, and F. J. Garcia-Vidal, 2008, Nat. Photonics 2, 175.

Williams, S. M., and J. V. Coe, 2006, Plasmonics 1, 87. 
Williams, S. M., A. D. Stafford, K. R. Rodriguez, T. M. Rogers, and J. V. Coe, 2003, J. Phys. Chem. B 107, 11871.

Williams, S. M., A. D. Stafford, T. M. Rogers, S. R. Bishop, and J. V. Coe, 2004, Appl. Phys. Lett. 85, 1472.

Wurtz, G. A., R. Pollard, and A. V. Zayats, 2006, Phys. Rev. Lett. 97, 057402.

Xie, Y., A. R. Zakharian, J. V. Moloney, and M. Mansuripur, 2005, Opt. Express 13, 4485.

Xing, Q., S. Li, Z. Tian, D. Liang, N. Zhang, L. Lang, L. Chai, and Q. Wang, 2006, Appl. Phys. Lett. 89, 041107.

Xu, T., X. Jiao, G. P. Zhang, and B. S., 2007, Opt. Express 15, 13894.

Yang, F., and J. R. Sambles, 2002, Phys. Rev. Lett. 89, 063901.

Ye, Y.-H., Z.-B. Wang, Y.-R. Cao, D. S. Yan, J.-Y. Zhang, X. Q. Lin, and T. J. Cui, 2007, Appl. Phys. Lett. 91, 251105.

Ye, Y.-H., Z.-B. Wang, D. S. Yan, and J.-Y. Zhang, 2007, Opt. Lett. 32, 3140.

Ye, Y.-H., and J. Y. Zhang, 2005, Opt. Lett. 30, 1521.

Yin, L., V. K. Vlasko-Vlasov, A. Rydh, J. Pearson, U. Welp, S.-H. Chang, S. K. Gray, D. B. Brown, and C. W. Kimball,
2004, Appl. Phys. Lett. 85, 467.

Yu, L. B., D. Z. Lin, Y. C. Chen, Y. C. Chang, K. T. Huang, J. W. Liaw, J. T. Yeh, J. M. Liu, C. S. Yeh, and C. K. Lee, 2005, Phys. Rev. B 71, 041405(R).

Yu, N., R. Blanchard, J. Fan, F. Capasso, T. Edamura, M. Yamanishi, and H. Kan, 2008, Appl. Phys. Lett. 93, 181101.

Yu, N., J. Fan, Q. J. Wang, C. Pfugl, L. Diehl, T. Edamura, M. Yamanishi, H. Kan, and F. Capasso, 2008, Nat. Photonics 2, 564.

Zayats, A. V., L. Salomon, and F. de Fornel, 2003, J. Microsc. 210, 344.

Zenneck, J., 1907, Ann. Phys. 23, 846.

Zhang, S., W. Fan, N. C. Panoiu, K. J. Malloy, R. M. Osgood, and S. R. J. Brueck, 2005, Phys. Rev. Lett. 95, 137404.

Zhou, L., and G. A. Kriegsmann, 2007, J. Acoust. Soc. Am. 121, 3288.

Zhou, R. L., X. S. Chen, S. W. Wang, W. Lu, Y. Zeng, H. B. Chen, H. J. Li, H. Xia, and L. L. Wang, 2008, Solid State Commun. 145, 23. 